\title{
Reclamation Techniques for Soils Treated with Non-Selective Residual Herbicides (Soil Sterilants)
}




\section{Alberta's Reclamation Research Program}

Regulating surface disturbances in Alberta is the responsibility of the Conservation and Reclamation Council. The Council Chairman is from Alberta Environmental Protection. The Council oversees a reclamation research program, established in 1978, to identify the most efficient methods for achieving acceptable reclamation in the province. Funding for the research program is provided by Alberta's Heritage Savings Trust Fund, Land Reclamation Program.

To assist with the development and administration of the research program, the Council appointed the inter-departmental Reclamation Research Technical Advisory Committee (RRTAC). Committee members represent the Alberta Departments of Agriculture, Food and Rural Development, Energy, and Environmental Protection, and the Alberta Research Council. The Committee updates research priorities, reviews research proposals, organizes workshops, and otherwise acts as ihe coordinating body for reclamation research in Alberta.

Additional information on the Reclamation Research Program may be obtained by contacting:

Chris Powter, Chairman

Reclamation Research Technical Advisory Committee

Alberta Environmental Protection

3rd Floor, Oxbridge Place

9820 - 106 Street

Edmonton, Alberta T5K 2J6

(403) $427-4147$

Additional copies of this report may be obtained at a cost of $\$ 5.00$ plus GST from:

Publication Services

Queen's Printers

11510 Kingsway Avenue

Edmonton, Alberta T5G 2 Y5

(403) $427-4952$

Prices quoted in the Reclamation Research Reports section of this Report do not include GST. Please make cheques payable to The Provincial Treasurer.

This report may be cited as:

M.M Cotton and M.P. Sharma, 1993. Reclamation Techniques for Soils Treated with Non-Selective Residual Herbicides (Soil Sterilants). Alberta Conservation and Reclamation Council Report No. RRTAC 93-12. ISBN 0-7732-6046-3. 84 pp. 


\section{Reclamation Techiques for Soils Treated with Non-Selective Residual Herbicides (Soil Sterilants)}

by

M.M. Cotton, Artemis Consulting Inc.

and

M.P. Sharma, Alberta Environmental Centre

Prepared for

Oil and Gas Reclamation Reseach Program

ALBERTA CONSERVATION AND RECLAMATION COUNCIL

(Reclamation Research Technical Advisory Committee) 
Digitized by the Internet Archive in 2015 


\section{Oil and Gas Reclamation Research Program}
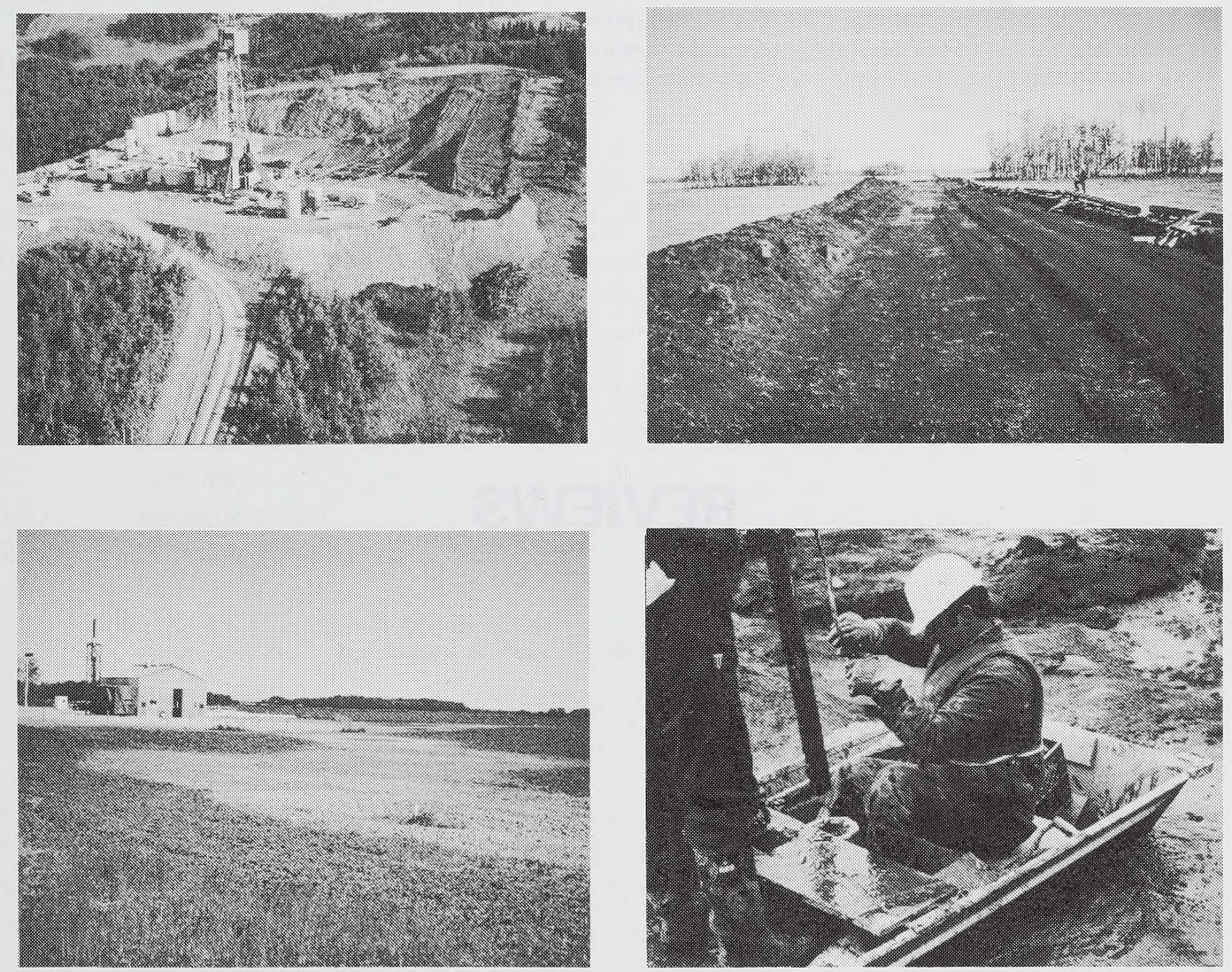

Members: Chris Powter (Chairman) - Alberta Environmental Protection; Roger Creasy - Energy Resources Conservation Board; Emil Dombroski - Alberta Environmental Centre; Al Fedkenheuer NOVA Corporation of Alberta; Jim Fujikawa - Alberta Environmental Protection; Arnold Janz - Alberta Environmental Protection; Alan Kennedy - Imperial Oil; Leon Marciak - Alberta Agriculture and Rural Development; Doug Mead - Shell Canada Resources Limited; Sam Takyi - Alberta Environmental Protection; Wayne Tedder - Alberta Agriculture, Food and Rural Development; Bill Wolff - Bisset Resource Consultants. 


\section{DISCLAIMER}

This report is intended to provide government and industry staff with up-to-date technical information to assist in the preparation and review of Conservation and Reclamation Approvals, and development of guidelines and operating procedures. This report is also available to the public so that interested individuals similarly have access to the most current information on land reclamation topics.

The opinions, findings, conclusions, and recommendations expressed in this report are those of the authors and do not necessarily reflect the views of government or industry. Mention of trade names or commercial products does not constitute endorsement, or recommendation for use, by government or industry.

\section{REVIEWS}

\section{Committee.}

This report was reviewed by RRTAC and the Oil and Gas Reclamation Reseach Program

\section{FUNDING}

This project was co-funded by the Alberta Heritage Savings Trust Fund, Land Reclamation Program through the Alberta Conservation and Reclamation Council, the Alberta Envrionmental Centre, and Imperial Oil Resources Ltd. RRTAC is grateful to Dr. M.P. Sharma, Alberta Environmental Centre and Mr. Dave Slade, Imperial Oil Resources Ltd. for arranging the funding. 


\section{TABLE OF CONTENTS}

LIST OF TABLES

Page vii

LIST OF FIGURES viii

ABSTRACT $\mathrm{X}$

ACKNOWLEDGEMENT $\mathrm{xi}$

1. INTRODUCTION 1

2.

OBJECTIVES 2

3.

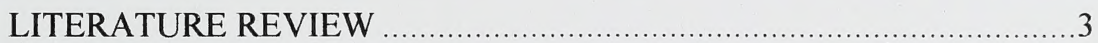

3.1

3.2

3.3

3.4

3.5

3.6

3.7

3.8

3.9

4. Soil Sterilants. 3 Activated Charcoal 4

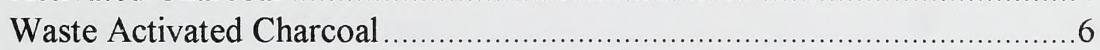

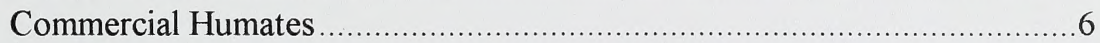

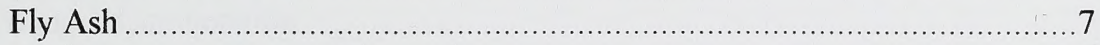

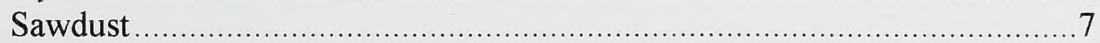

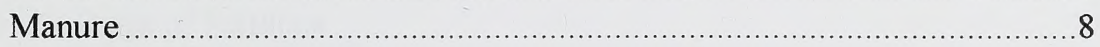

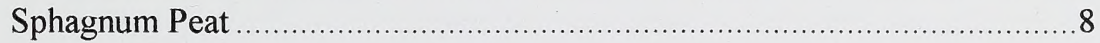

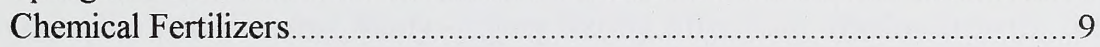

4.1.

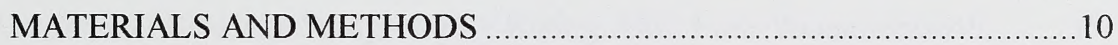

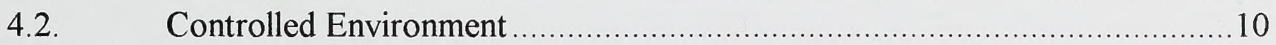

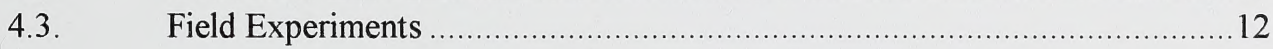

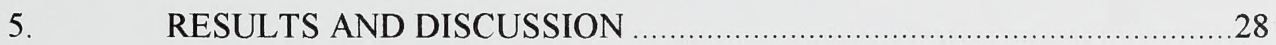

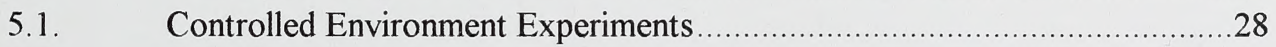

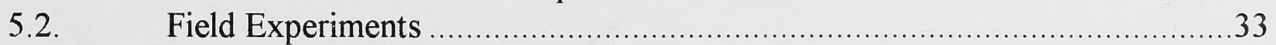

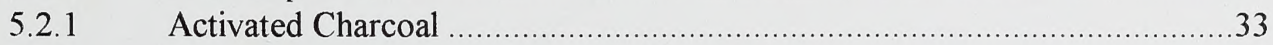

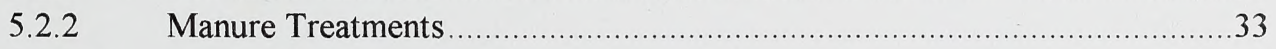

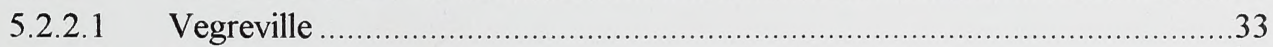

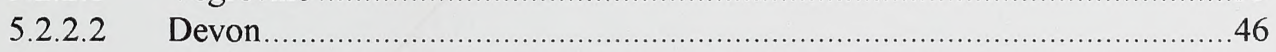

5.3. Well Composted Manure vs Peat plus Fertilizer Treatments ......................48

5.4. Five Year Old Manure vs. Ten Year Old Manure …................................50

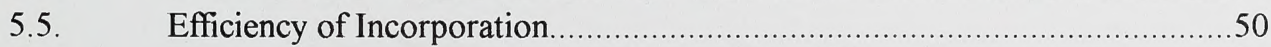

5.6. Effect Of Amendments On Soil Physical Properties ...................................56

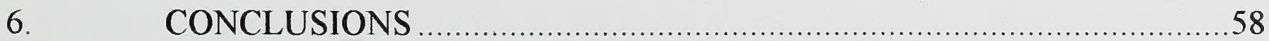

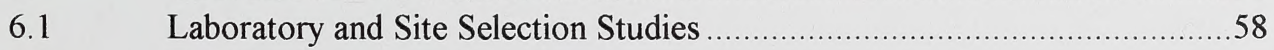

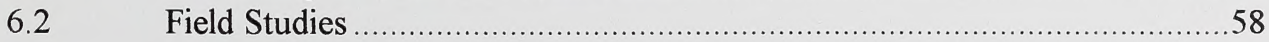




\section{TABLE OF CONTENTS}

Page

7

FUTURE RESEARCH

61

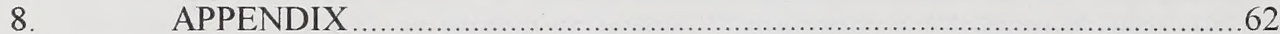

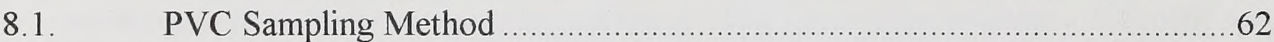

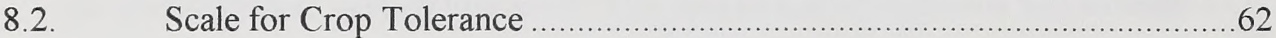

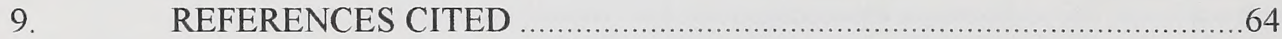




\section{LIST OF TABLES}

Table

Page

1. Soil Sterilant Summary. 3

2. Soil Chemical and Physical Properties 10

3. Levels of Herbicide Residues in the Devon Soil as Determined by HPLC

4.

Amendments 13

5. Rates of Amendments in the Devon Soil 14

6. Rates of Amendments for Vegreville Soil 15

7. Activated Charcoal Field Rates 25

8. Manure Composition 25

9.

Field Rates of Fertilizer 27

10. Vegreville Soil Bioassay Study - Injury Rating After Four Weeks Growth...32

11. Devon Soil Bioassay Study - Injury Rating After Four Weeks Growth 35 


\section{LIST OF FIGURES}

Figure

1. 1991 Vegreville Field Plots - Bromacil

2. Vegreville Field Plots - Tebuthiuron

3. Vegreville Field Plots - Bromacil.

4. Vegreville Field Plots - Tebuthiuron

5. Devon 1991 Field Plots

7. Devon 1992 Field Plots

8. Devon 1992 Field Plots - 5 year old and 10 year Old Manure Comparison...24

9. Application of Activated Charcoal with a Tractor Mounted Spray Unit to 15 to $30 \mathrm{~cm}$ Soil Depth at Devon.

10. Application of Manure Using Small Bulldozer to 15 to $30 \mathrm{~cm}$ Depth Soil at Devon 26

11. Rotovating the 15 to $30 \mathrm{~cm}$ Soil Depth at Devon 28

12. Stripping the Top $15 \mathrm{~cm}$ of Soil at Devon by block .28

13. Replacing the Top $15 \mathrm{~cm}$ of Soil with the Loader (right) 30

14. Bioassay Experiment Using the Vegreville Soil with Tebuthiuron Residues at $2.4 \mathrm{ppm}$......

15. Bioassay Experiment Using the Vegreville Soil with Tebuthiuron Residues at $0.8 \mathrm{ppm}$

16. Treatment Comparison on the Vegreville Soil with Initial Bromacil

Residues of $2 \mathrm{~kg} / \mathrm{ha}$.

17. Treatment Comparison on the Vegreville Soil with Initial Bromacil

Residues of $6 \mathrm{~kg} / \mathrm{ha}$ 38

18.

Photograph of Crops. 


\section{LIST OF FIGURES (Concluded)}

Figure

19. Treatment Comparison on the Vegreville Soil with Initial Bromacil

Residues of $2 \mathrm{~kg} / \mathrm{ha}$.

20. Treatment Comparison on the Vegreville Soil with Initial Bromacil

Residues of $6 \mathrm{~kg} / \mathrm{ha}$.

21. Treatment Comparison on the Vegreville Soil with Initial Bromacil

Residues of $2 \mathrm{~kg} / \mathrm{ha}$.

22. Treatment Comparison on the Vegreville Soil with Initial Bromacil

Residues of $6 \mathrm{~kg} / \mathrm{ha}$ 43

23. Treatment Comparison on the Devon Soil 44

24. Photograph of Crops 45

25. Treatment Comparison on the Devon Soil. 46

26. Treatment Comparison on the Devon Soil.

27. Photograph of Crops. 48

28. Photograph of Crops

29. Treatment Comparison on the Vegreville Soil with Initial Tebuthiuron

Residues of $2 \mathrm{~kg} / \mathrm{ha}$

30. Treatment Comparison on the Vegreville Soil with Initial Tebuthiuron

Residues of $6 \mathrm{~kg} / \mathrm{ha}$....... 54

31. Treatment Comparison on the Devon Soil .55

32. Treatment Comparison on the Devon Soil 56

33. Treatment Comparison on the Devon Soil. .57

34. Treatment Comparison on the Devon Soil. .58

35. Illustration of a PVC Tube for Bioassays. 


\section{ABSTRACT}

Soil sterilants -- non-selective residual herbicides -- have been extensively used in Alberta for total vegetation control around oil and gas wells, gas processing plants, and rights-ofway. Problems with such sterilants occur when a treated site is no longer viable for industrial use and the soil must be restored to its original productive state. The aim of this project was to examine several amendments (activated charcoal, manure, sawdust, waste activated charcoal, fly ash, commercial humates, commercial microbes, peat, and fertilizer) for their effectiveness in reclaiming herbicide residues in soil under controlled environment and field conditions. The influence of amendment to herbicide ratio, soil type, depth of herbicide contamination, and number of incorporations on the effectiveness of the amendments was studied. The experiments were set up at Vegreville using a Chernozemic black silt loam soil treated with bromacil or tebuthiuron at rates of 2 and $6 \mathrm{~kg} / \mathrm{ha}$ active ingredient, and at Devon on a loamy sand sub-soil with phytotoxic atrazine and diuron residues as high as $5 \mathrm{~kg} / \mathrm{ha}$ active ingredient.

Activated charcoal applied at rates as low as $200 \mathrm{~kg}$ per $1 \mathrm{~kg}$ of active ingredient provided effective protection from herbicide injury to oats (Avena sativa L.), alfalfa (Medicago sativa L.), and bromegrass (Bromus inermis). As the ratio of activated charcoal increased above 200:1 no significant difference was evident between the treatments. Activated charcoal (200:1, and $300: 1)$ in combination with well-composted manure (1:1 manure to soil by volume) provided protection from herbicide injury and resulted in greater crop biomass than either charcoal or manure alone. The quality of manure affected its ability to prevent herbicide injury; wellcomposted manure alone protected crops against herbicide injury on both soils. Sphagnum peat (1:1 peat to soil by volume) plus fertilizer may be substituted as an amendment for manure, on the loamy sand soil. Fertilizer rates varied with manure source and composition: N, 91 to $212 \mathrm{~kg} / \mathrm{ha}$; $\mathrm{P}_{2} \mathrm{O}_{5}, 412$ to $697 \mathrm{~kg} / \mathrm{ha}$; and $\mathrm{K}_{2} \mathrm{O}, 1622$ to $2254 \mathrm{~kg} / \mathrm{ha}$. 


\section{ACKNOWLEDGMENTS}

Funding for the project was provided by the Alberta Heritage Savings Trust Fund Land Reclamation Program that is administered by the Reclamation Research Technical Advisory Committee of the Alberta Land Conservation and Reclamation Council; the Alberta Environmental Centre; and Imperial Oil Resources Limited.

The supervision and guidance in this project from Dr. W.H. Vanden Born, and Dr. W.B. McGill from the University of Alberta is appreciated. Thanks to Ms. Arden Alexander for her assistance with the field work. We would also like to thank Dr. R.T. Hardin for helping with the data analysis.

The Alberta Environmental Centre (AEC) provided the facilities for this research. Thanks to the many people from the Alberta Environmental Centre who helped with this project. A special thanks to Mr. Harold Fedema, Mr. Jeff Newman, Mr. Paul Yeung, and Mr. Spencer Lofthaug. We would also like to thank Dr. Zack Florence for his assistance with the experimental design for the field study.

The technical assistance provided by Ms. Teresa Kupchenko, Ms. Fiona Salkie, Mr. Michael Splinter, Ms. Leslee Porayko, Ms. Cynthia Cotton, Ms. Lori Wood, Ms. Debbie Forster, Ms. Carly Forster, and Mr. Brad Mills from Artemis Consulting Inc. is appreciated.

Thanks to Alberta Agriculture for the use of their soil sampling unit, and to Mr. Gary McGregor for his technical assistance. Thanks to Ms. Denise Maurice for her encouragement, and for the huge stack of literature she provided for the study.

Thanks to Moen Lumber Sales (Sawdust), Edmonton, Alberta; Good Earth Environmental Research Corp. (Commercial humates), Calgary, Alberta; Humate Canada Limited (Commercial humates), Calgary, Alberta; Chemicals Specialties Int. (Superbugs), California; TransAlta Utilities (fly ash); Alberta Power Ltd. (fly ash); Mr. Tjerk Wartena (10 year old manure), Riley, Alberta; Sun Grow Horticulture Canada Ltd. (Peat), Seba Beach, Alberta; Dupont (Hyvar $\AA$ ); and DowElanco (Spike ${ }^{\circledR}$ ) for donation of materials.

Thanks to Mr. Chris Powter, Ms. Clara Qualizza, and Mr. Bradley Jones for their support, advice, and thoughtful criticism. 

Soil sterilants -- non-selective residual herbicides -- have been used extensively in Alberta for total vegetation control on oil and gas wells, gas processing plants, rights-of-way, railways, saw mills, pulp mills, and electrical utility sites. They are used to maintain fire guards around facilities, to control noxious weeds in response to agricultural concerns, and to reduce weeds that might create an employee working hazard. The sterilants commonly used in Alberta are atrazine, bromacil, tebuthiuron and diuron. It is estimated that there may be as many as 61,750 oil and gas sites in Alberta with soil sterilant residues (Cotton and Sharma 1992). Bromacil and tebuthiuron were identified as the herbicides most commonly affecting sites now being reclaimed in Alberta (Cotton and Sharma 1992). Atrazine and diuron were the most commonly used herbicides in Alberta from 1986 to 1990, and thought to be of concern on future reclamation sites (Cotton and Sharma 1992).

By design, such herbicides applied at industrial rates can leave soil devoid of vegetation for years depending on the type and rate of herbicide used and the soil and climatic conditions. Furthermore, products applied from a shaker can as pellets or granules may facilitate application at higher than recommended rates to obtain longer-term control. An industry practice of maintaining bare ground on a site usually involves repeat applications of one or more products every three to five years. A build up of herbicide residues reaching a depth of $45 \mathrm{~cm}$ or more can result.

Herbicide residues become a problem when they leach from a target area, or when a site is no longer required for industrial use and the residues must be deactivated so the soil can be re-vegetated. This project was initiated to examine several amendments (activated charcoal, manure, sawdust, waste activated charcoal, fly ash, commercial humates, commercial microbes, peat, and fertilizer) for their effectiveness in reclaiming herbicide residues. Two soil types were selected for the study: (1) a chernozemic black silt loam soil treated with bromacil or tebuthiuron at rates of 2 and $6 \mathrm{~kg} / \mathrm{ha}$ active ingredient; and (2) a loamy sand sub-soil with phytotoxic atrazine and diuron residues as high as $5 \mathrm{~kg} / \mathrm{ha}$ active ingredient.

Controlled environment experiments were set up to screen the amendments using oats and alfalfa as indicator species. Amendments and rates selected for field trials were based on the results of the controlled environment experiments. The influence of amendment to herbicide ratio, soil type, depth of herbicide contamination, and number of incorporations on the effectiveness of the amendments was studied. Oats, alfalfa, and bromegrass were used as the indicator species for the field work. Following the results of the first years field experiments, additional field experiments were added in an attempt to explain the effect of manure quality. 
The objectives of this project were:

1. To examine the use of several soil amendments for the inactivation of herbicide residues under controlled environment and field conditions.

2. To investigate the influence of herbicide and amendment ratios, soil type, depth of herbicide contamination, and number of incorporations on the effectiveness of the amendments.

3. To demonstrate reclaimed soil quality and productivity through vegetation establishment.

4. To identify the extent of sterilant contamination on an industrial site. 
3. LITERATURE REVIEW

Atrazine, bromacil, tebuthiuron, and diuron are primarily soil active herbicides used for general vegetation control on non-crop land. General information about the herbicides and their characteristics is presented in Table 1.

Table 1. Summary of soil sterilant characteristics.

\begin{tabular}{lllll}
\hline \multirow{2}{*}{ Trade names } & Atrazine & Bromacil & Diuron & Tebuthiuron \\
\cline { 2 - 5 } & Aatrex ${ }^{\circledR}$ & Hyvar ${ }^{\circledR}$ & Karmex ${ }^{\circledR}$ & Spike ${ }^{\circledR}$ \\
& Primatol ${ }^{\circledR}$ & $\begin{array}{l}\text { Krovar } \\
\text { Calmix }\end{array}$ & Krovar ${ }^{\circledR}$ & Herbec ${ }^{\circledR}$
\end{tabular}

Industrial

$11.25-22.5$

$10.7-21.5$

$11.2-44$

$4.4-11.25$

application rate

( $\mathrm{kg} / \mathrm{ha}$ a.i.)*

Solubility in water ${ }^{\mathrm{a}} 33$ ppmw

815 ppmw

42 ppmw

$2300 \mathrm{ppmw}$

Soil adsorption

149

72

400

620

coefficient $-\mathrm{K}_{\mathrm{Oc}} \mathrm{b}^{\mathrm{b}}$

Leaching potential ${ }^{\mathrm{a}}$ medium

high

low

medium

Half life in soil

$24-32+$

$20-24$

$52-78$

$52-60$

(weeks) $^{\mathrm{a}}$

* a.i. (active ingredient)

a WSSA 1989

b Kenaga 1980

Herbicides can be deactivated in soil with amendments. The mechanisms of inactivation of the amendments are adsorption (sorption) and degradation or both. Adsorption is a physical or chemical binding that can be achieved by the addition of an amendment with a high adsorptive capacity for organics (for example activated charcoal). Experimentally, adsorption is usually determined by chemical loss from solution; therefore it might be more appropriately replaced by the general term sorption. Sorption refers to the retention process with no 
distinction between the specific processes of adsorption, absorption and precipitation (Koskinen and Harper 1990).

Degradation can be through biological, abiotic, photolysis, or volatilization processes. According to the Weed Science Society of America (WSSA 1989) the primary degradation of all the above herbicides is microbial, although Howard (1991) reports that abiotic degradation may also play an important role for atrazine. Degradation of these herbicides due to volatilization or photo-decomposition is probably negligible (WSSA 1989). Some degradation of the herbicides will occur naturally, but reclamation efforts usually try to enhance degradation by the addition of organic material such as manure and peat. Not only does organic material in soil enhance degradation, but it has been well established that organic material acts as a primary sorbent for pesticides (Guo et al. 1991a,b).

Animal manures or organic matter of any kind should be helpful in reducing herbicide residues from soils, especially soils naturally low in organic matter (Ashley and Rahn 1967). The most readily available and commonly used soil amendment with high adsorptive properties is organic humus in the form of decayed plant or animal matter, manure, or sewage sludge (Shea 1985).

\section{ACTIVATED CHARCOAL}

Activated charcoal (carbon) is probably the most widely investigated adsorbent for herbicide detoxification (Hoagland 1989; Lamoreaux et al. 1989). The effectiveness of activated charcoal in the adsorption of herbicides is related to the surface area and the number and size of the pores. Typically, the surface area of activated charcoal can range from 500 to $1400 \mathrm{~m}^{2} / \mathrm{g}$ (Coffey and Warren 1969; Hoagland 1989). Charcoal is available in two main types: granular $(>300 \mu \mathrm{m}, 50$ mesh or greater) and powdered $(<300 \mu \mathrm{m})$. Charcoal adsorption capacity is also dependent on total surface area while the rate of adsorption is related to particle size (Hoagland 1989 and references cited there in). Adsorption is influenced by the material from which the activated charcoal is made and on the method of activation (Coffey and Warren 1969).

Although not all forms of activated charcoal absorb equally, little work has been done exploring how different types of charcoal adsorb particular herbicides (Hoagland 1989; Jordan and Smith 1971). Coffey and Warren (1969) studied five activated carbons for their relative efficiency adsorbing trifluralin; Hydro Darco B ${ }^{\circledR}$ and Darco $M{ }^{\circledR}$ (lignite charcoals) were found to be the most efficient. Jordan and Smith (1971) compared 24 different charcoals found that no one criterion (eg. particle size, surface area, $\mathrm{pH}$ ) appeared to be responsible for differences 
between all charcoals. MCB Petroleum Base charcoal (plus 100 mesh) was found to be the most efficient in their study.

Hartley and Sutcliff, Speakman and Co. were the first to use activated charcoal as an adsorbent for herbicides when they patented it in Britain as a seed coating to protect seedlings against injury from pre-emergent herbicides (Hoagland 1989). Since that time, activated charcoal for the purpose of deactivating herbicides has been used in many ways including root dipping, banding applications, surface applications, and the one most applicable to this research, soil incorporated applications. Ratios of activated charcoal to herbicide active ingredient required to inactivate herbicide residues vary in the literature from 15:1 to 3600:1. LeBaron (1970) cites ratios of charcoal to herbicide as low as 15:1 for the inactivation of atrazine residues in an oat crop. Ahrens (1965) suggested that as a general rule a ratio of 200:1 of finely ground activated charcoal to triazine level remaining in the soil could be used. For higher residue levels or when very sensitive crops are involved, ratios of 400:1 may be required. Anderson (1968) cited a range of 200:1 to 400:1 of charcoal to simazine depending on the soil organic matter content, and the efficiency of incorporation. Shoup and Whitcomb (1984) used ratios of activated charcoal to bromacil (active ingredient) of 22:1, 66:1, and 109:1. They found that these levels were not adequate to prevent herbicide damage on a clay loam soil. Lamoreaux et al. (1989) used rates of charcoal to norflurazon of approximately 100:1,200:1, and 300:1 to reduce herbicide injury to cotton, soybean and corn, respectively from a sand matrix. In a greenhouse study with silty clay (tropical) soil charcoal to herbicides ratios as low as 66:1 (propazine) were found to be effective in preventing herbicide injury in beans, but ratios of 3600:1 were required to prevent picloram injury. In the field, ratios of approximately 240:1 did not completely prevent propazine and picloram injury to cucumbers or beans (Bovey and Miller 1969). These differences in ratios can be attributed to variations in herbicide, sensitivity of test crops, soil texture and organic content, and the efficiency of incorporation of the carbon (Ogg 1982; Sharma 1989). In a greenhouse study, Andersen (1968) found the amount of activated charcoal required depends on the organic matter content and the efficiency of incorporation and therefore thorough incorporation would be a major issue in using the technique in the field. A greenhouse study by Sharma (1989) found that protection of oat plants from herbicide injury was slightly better under lower moisture regimes (below field capacity) than at the high moisture regimes (field capacity), particularly on lower herbicide:charcoal ratios (100:1, and 200:1).

Field research is particularly important in Alberta where information is lacking on how Alberta conditions will influence the effectiveness of activated charcoal on soil sterilant residues in Alberta (Sharma 1989). Harvey (1973) reported that exposure to certain environmental conditions (e.g., cycles of freezing to $-27^{\circ} \mathrm{C}$, and thawing to $25^{\circ} \mathrm{C}$ ) exhibited a reduction in the adsorptive capacity of the activated carbon, and may release atrazine with the potential of injuring 
susceptible crops long after the original herbicide application. These results need to be explored under Alberta conditions.

\section{WASTE ACTIVATED CHARCOAL:}

Waste (spent) activated charcoal is available in large quantities from food processing plants, sugar refineries and breweries where it is used for purification. One Calgary, Alberta distillery currently disposes of approximately 1 tonne a week into a sanitary landfill site. Use of these wastes for soil reclamation is desirable because alternative disposal methods such as landfill and incineration are more expensive and have greater potential for environmental contamination (Guo et al. 1991a).

Gou and co-workers (1991a,b) looked at waste activated charcoal obtained from filtration processing of corn sweetener to decrease leaching of atrazine in soil columns (1991b), and to deactivate atrazine residues using sandy soil in a greenhouse study (1991a). In the leaching study they found atrazine leaching from coarse-textured soil could be significantly reduced by the waste activated charcoal, and the reduction was associated with significant increases in sorption and degradation. In the deactivation study loading rates of $2100 \mathrm{~kg}$ of total carbon/ha $(95.4 \%$ total carbon content) were used. It was found that the waste activated charcoal had a significant inhibitory effect on atrazine and alachlor residue levels as high as 4 ppm (approximately 9.6 $\mathrm{kg} / \mathrm{ha})$.

A review by LeBaron (1970) discussed the use of waste activated charcoal for herbicide deactivation. Although the material was acknowledged to be extremely cheap, preliminary studies indicated that the carbon was relatively ineffective at practical rates.

\section{COMMERCIAL HUMATES}

Humic acid, a component of the organic matter fraction of soil, has been found to be an important adsorbent for herbicides, decreasing their biological availability in soil and their potential for environmental pollution (Saint-Fort and Visser 1988). Enhanced binding of pesticides to humic substances has been considered as a method of soil reclamation (Bollag 1991; Schnitzer no date).

Commercial humates are materials mined for their humic acid and other organic acid content. They are either oxidized lignites and the products derived from them or products of weathered subbituminous coal seams and carbonaceous shales. They are an earthy, mediumbrown, coal-like substance high in humic acid (but usually low in fulvic acid) mined in North 
Dakota, Texas, New Mexico, Idaho, and elsewhere (Stevenson 1986). There are known sources of commercial humates in Alberta and Saskatchewan, but there is no active production of humic acid-bearing materials in Canada at present (Hoffman et al. 1993). Commercial humates are being imported from the United States and marketed for use as organic soil amendments on the basis of their beneficial effects to soil physical, chemical, and biological properties.

\subsection{FLY ASH}

Fly ash is the solid material which is carried away in the flue gas from the power plant boiler during coal combustion (El-Mogai et al. 1988). Fly ash has been shown to have beneficial effects on crop yield and development (El-Mogazi et al. 1988; Gwyer and Robertson 1979; Macyk 1983; Page et al. 1979; Pawar and Dubey 1986), but it has also been shown to have detrimental effects, particularly due to excessive boron and selenium levels (Aitken and Bell 1985; El-Mogazi et al. 1988; Gwyer and Robertson 1979). It has been found that fly ash can increase the water holding capacity of soil (Campbell et al. 1983), and that it can be used as an acid neutralizing amendment to acidic soils (El-Mogazi et al. 1988; Gwyer and Robertson 1979; Taylor and Schuman 1988).

Vandenbusch and Sell (1992) report fly ash to be an effective sorbent, capable of removing many organic contaminants due to its large surface area and its high residual carbon content. They found fly ash to be useful for removing organics from a small municipal waste treatment facility effluent. Of particular interest to this study is a laboratory study by Albanis and co-workers $(1988,1989 \mathrm{a}, \mathrm{b})$, who found that small amounts of fly ash $(0.1 \%$ to $0.5 \%)$ were able to increase the soils capacity to detoxify atrazine, lindane, and methyl parathion residues in a clay and a sandy clay loam soil. However, this was found to be a function of increased degradation caused by hydrolysis, due to the alkaline $\mathrm{pH}$ values from the addition of fly ash to the soil, rather than adsorption of the pesticides. Albainis and co-workers actually found that the adsorption of the pesticides decreased with the addition of the fly ash. This work illustrates that fly ash requires further investigation for use in detoxifying herbicide residues.

\section{SAWDUST}

Sawdust was chosen as an amendment because it is generally a widely available, inexpensive organic material. Sawdust incorporation was found to significantly increase the organic matter content of soil (Webster 1961). It is capable of improving the aeration of compacted industrial sites, and increasing soil moisture (Webster et al. 1957); all of which should 
enhance the microbial population, and aid in herbicide degradation and inactivation. Nitrogen fertilizer must be included with sawdust to prevent nitrogen deficiency. Douglas fir, Canada hemlock, balsam fir and white pine have all been reported as suitable types of sawdust for agricultural use (Webster et al. 1957).

3.7 MANURE

Degradation of herbicides in soil was promoted by the addition of manure (DuahYenumi and Kuwatsuka 1982). The degradation rate increased with increasing amounts of manure, however the underlying factors which caused the difference were unclear. It was speculated that the addition of organic materials quickly increases the microbial numbers and activity, and after the depletion of the additives, some of the organisms begin to attack the less desirable carbon source furnished by the pesticides. Ndayegamiye and Cote (1989) reported that soil microflora populations (bacteria, fungi, actinomycetes, ammonifiers and nitrifiers) were greatly improved by the addition of solid cattle manure at rates of 20,40 and $60 \mathrm{Mg} / \mathrm{ha}$. The addition of manure to soil increases the humic acid content of the soil (Campbel et al. 1986). There are many instances of herbicides being reported to adsorb to humic acid (Bollang 1991; Klowkowski and Fuhr 1987; Saint-Fort and Visser 1988). It is therefore possible that manure contributes to decreased herbicide phytotoxicity by adsorption and degradation.

\section{8}

\section{SPHAGNUM PEAT}

Peat moss had been reported to reduce simazine injury to oats (Grover 1966). There are a number of properties that may be responsible for decreased herbicide phytotoxicity. Logan (1978) and references cited therein report peat to have a relatively high cation exchange capacity ranging from $50 \mathrm{me} / \mathrm{L}$ to $300 \mathrm{me} / \mathrm{L}$ depending on the stage of decomposition. This would indicate a potential for herbicide adsorption. Sphagnum peat moss is able to increase the water holding capacity of soil by adsorbing between 8 and 30 times its dry weight (Feustel and Byers 1936; Grandmaison 1982), and it contains a large proportion of substances that readily decompose and therefore should support large amounts of microbial activity (Logan 1978). Peat is not known to be a supplier of major plant nutrients. Logan (1978) and references cited therein report total nitrogen to range from $0.7 \%$ to $3.7 \%$ by weight. 
The microbial degradation of benthiocarb, MCPA and 2,4-D herbicides in soil was promoted by the addition of chemical fertilizers in a laboratory study (Duah-Yentumi and Kuwatsuka 1981, 1982). The degradation of MCPA, and 2,4-D was found to be stimulated more by $\mathrm{P}$ than either $\mathrm{N}$, or $\mathrm{K}$. 
4.1, SOILS

Four sites were evaluated for their potential use in the research study. Two sites were selected. One in Vegreville at the Alberta Environmental Centre (chernozemic black silt loam soil), and another at Imperial Oil Resources Limited in Devon (loamy sand sub-soil). The Vegreville site was located on cultivated land containing no herbicide residues. It was established to examine inactivation of known amounts of selected herbicides.

The Devon site was located in the equipment storage yard of the gas processing plant and had been treated with repeated applications of herbicides over many years. From the lack of vegetation, all the surface soil had eroded away, exposing the sub-soil material. Soils from the vacinity are orthic black chernozems. This site is an older oil and gas field and contains many sites that will be decommissioned in the next few years. One of several companies in the area, Imperial Oil Resources, will be looking at decommissioning approximately 800 locations. The major chemical and physical properties of the two soils are listed in Table 2.

Table 2. Soil chemical and physical properties.

\begin{tabular}{llll}
\hline PROPERTY & \multicolumn{2}{c}{ DEVON SOIL } & VEGREVILLE SOIL \\
\hline & $(0-15 \mathrm{~cm})$ & $(15-30 \mathrm{~cm})$ & $(0-15 \mathrm{~cm})$ \\
& SUB-SOIL & SUB-SOIL & TOP SOIL \\
\hline pH $\quad 8.4$ & 8.3 & 5.7 \\
EC (mS/cm) & 0.40 & 0.33 & 0.23 \\
Sol. Ca (ppm) & 40 & 40 & 7 \\
$\quad \mathrm{Na}(\mathrm{ppm})$ & 13 & 11 & 50 \\
$\quad \mathrm{~K} \mathrm{(ppm)}$ & 6 & 6 & 5 \\
$\quad \mathrm{Mg}(\mathrm{ppm})$ & 5 & 5 & 5 \\
SAR & 0.5 & 0.5 & 3.5 \\
Textural class & LS & LS & SiL \\
Sand (mg/kg) & 81 & 84 & 24 \\
Silt (mg/kg) & 9 & 7 & 54 \\
Clay (mg/kg) & 10 & 9 & 22 \\
O.M. (mg/kg) & 0.7 & 0.4 & 10 \\
Water repellency (MED) & 0.0 & 0.0 & 0.0 \\
\hline
\end{tabular}

4.2. CONTROLLED ENVIRONMENT

The Vegreville soil was collected to a $15 \mathrm{~cm}$ depth (approximately $120 \mathrm{~kg}$ ) with a back hoe, and stored at room temperature (prior to herbicide treatment). After being air-dried 
and sieved to remove stones the soil was mixed with bromacil $\left(\mathrm{Hyvar}^{\circledR} \mathrm{X}, 80 \%\right.$ wettable powder) or tebuthiuron (Spike ${ }^{\circledR}, 80 \%$ wettable powder) to obtain concentrations of 0.8 , and 2.4 ppm active ingredient. The low rate represents carryover that might result from a single application after one or more years, and the high rate is representative of a build up from several applications. A batter beater "Model 130T" (trademark of Blakerlee Food Service Equipment Inc., Scarborough, Ontario) was used to incorporate the herbicide. Each sample was mixed for 15 minutes. Following herbicide incorporation the soil was stored at $4^{\circ} \mathrm{C}$.

At the Devon site ten sample locations were chosen using a grid system in the $2100 \mathrm{~m}^{2}$ proposed plot area. There was no vegetation establishment on the proposed plot area therefore no areas of high or low residue concentrations were discernible. Four $10 \mathrm{~cm}$ by $60 \mathrm{~cm}$ cores were collected at each of the ten locations. Four cores were taken to collect sufficient soil. The cores were split into $15 \mathrm{~cm}$ increments, and equivalent increments were composited. A control location (unaffected by soil sterilants) was chosen adjacent to the sampling area. The samples were then stored in plastic bags in the freezer at $-20^{\circ} \mathrm{C}$ (samples were collected in the winter under conditions of frost and snow).

Sub-samples were sent to a private laboratory where they were extracted, partitioned with dichloromethane (DCM) and analyzed by HPLC using UV detection at $254 \mathrm{~nm}$ to determine herbicide residues remaining in the soil (Table 3).

Table 3. Levels of herbicide residues (active ingredient) in the Devon soil as determined by HPLC.

\begin{tabular}{lllll}
\hline SAMPLE DEPTH & DIRUON & ATRAZINE & TOTAL & ADJUSTED TOTAL* \\
\hline $0-15 \mathrm{~cm}$ & $0.03 \mathrm{ppm}$ & $1.60 \mathrm{ppm}$ & $1.63 \mathrm{ppm}$ & $\mathbf{2 . 1 8} \mathbf{~ p p m}$ \\
$15-30 \mathrm{~cm}$ & $0.03 \mathrm{ppm}$ & $0.84 \mathrm{ppm}$ & $0.87 \mathrm{ppm}$ & $\mathbf{1 . 1 7} \mathbf{~ p p m}$ \\
$30-45 \mathrm{~cm}$ & $0.02 \mathrm{ppm}$ & $0.51 \mathrm{ppm}$ & $0.53 \mathrm{ppm}$ & $\mathbf{0 . 7 1} \mathbf{p p m}$ \\
$45-60 \mathrm{~cm}$ & $0.01 \mathrm{ppm}$ & $0.56 \mathrm{ppm}$ & $0.57 \mathrm{ppm}$ & $\mathbf{0 . 7 6} \mathbf{p p m}$ \\
$\begin{array}{l}\text { Minimum } \\
\text { detectable level }\end{array}$ & 0.01 & 0.04 & & \\
\% Recovery & $70 \%$ & $75 \%$ & & \\
\hline
\end{tabular}

* Total has been adjusted for percent recovery.

Whole plant bioassays, using oats (Avena sativa $\mathrm{L}$.) $\mathrm{cv}$. Calibre and alfalfa (Medicago sativa $\mathrm{L}$.) $\mathrm{cv}$. Beaver as indicator species, were conducted to study the effects of various amendments on the inactivation of herbicide residue on the two soils. These were selected as representative of monocotyledon and dicotyledon crops. Activated charcoal, manure, activated charcoal plus manure, sawdust, two commercial humates, a commercial microbial solution, fly ash 
and waste activated charcoal were investigated for effectiveness in reducing herbicide toxicity. Amendment details and rates are presented in Table 3, 4, 5, and 6.

The Devon soil was air-dried overnight and then sieved (3-mm mesh) to remove stones. Amendments were incorporated by measuring soil and amendment into a heavy plastic bag filled with air. The bags were sealed and shaken for 3 minutes. Approximately $500 \mathrm{~g}$ Devon or $330 \mathrm{~g}$ Vegreville soil/amendment mix was then poured into $450 \mathrm{~mL}$ cups. The absence of drainage holes in the pots prevented loss of herbicide by leaching (Harvey 1973).

Each cup was immediately watered with $90 \mathrm{~mL}$ of water. The cups were seeded 1 week later with 7 oat seeds or 10 to 12 alfalfa seeds per cup. They were planted to a depth of approximately $1 \mathrm{~cm}$ below the surface of the soil. The cups were set into growth chambers under a $22^{\circ} \mathrm{C}$ for $16 \mathrm{~h}$ and at $16^{\circ} \mathrm{C}$ for $8 \mathrm{~h}$ temperature regime. The soil in each container was brought to field capacity following seeding. For the remainder of the experiment the moisture content was maintained between $65 \%$ and $100 \%$ of field capacity by watering daily or twice daily as required. Upon emergence, and 2 weeks later the cups were fertilized (50 mL/cup) with a 100 ppm N solution. All cups were arranged in a randomized split/block design with four replicates.

Approximately 1 week after emergence, seedlings were thinned to three uniform plants in each container. The plants were scored and photographed in the second and fourth week. Fresh and dry weight of the aerial biomass was recorded after four weeks. Data were analyzed by ANOVA and Duncan's multiple range. The specific test was "PROC ANOVA" with the "MEANS/DUNCAN" option.

\subsection{FIELD EXPERIMENTS}

All experimental plots were 2.5 or $3 \mathrm{~m} \mathrm{x} 6 \mathrm{~m}$ in size to allow for equipment traffic, however only the center $2 \times 6 \mathrm{~m}$ of the plots received the amendment and/or the herbicide application. The Devon experiments were set up in a randomized complete block design with 4 replicates. At Vegreville plots were sprayed with 2 and $6 \mathrm{~kg} / \mathrm{ha}$ of bromacil or tebuthiuron in fall 1990 or 1991 to provide phytotoxic residues in the soil for subsequent years. They were set up in a split plot design with 4 replicates.

Amendment rates for the field trials were based on the results of the laboratory studies. Following the results of the first year, additional trials were added. Figures 1 to 4 show the plot layouts for the Vegreville site. Figures 5 to 8 show the Devon layouts. 
Table 4. Description of amendments used in controlled environment study

Charcoal: $\quad$ NORIT ${ }^{\circledR}$ activated charcoal 100 plus mesh size. A trademark of Atlas Chemical Co. obtained from Van Waters \& Rogers, Edmonton.

Manure: $\quad$ Composted Steer Manure ${ }^{\circledR}$ manufactured by Cattleland Feed Yards Ltd.

Distributed by Westland Distributors, Calgary, Alberta.

Minimum analyses of the manure:

Total $\mathrm{N}$ $0.6 \%$

Avail. $\mathrm{P}_{2} \mathrm{O}_{5}$ $0.5 \%$

Soluble $\mathrm{K}_{2} \mathrm{O}$ $0.6 \%$

Minimum O.M $20 \%$

Max. Moisture $50 \%$

Sawdust: $\quad$ Spruce and poplar sawdust from Moen Lumber Sales, Edmonton, Alberta.

Humate 1: An organic (humic) product from Good Earth Environmental Research Corp., Calgary, Alberta.

Humate 2: Humus ${ }^{\circledR}$ an organic product from Humate Canada Limited, Calgary, Alberta.

Contains:

Humic and fulvic acids $65 \%$ Inorganic salts $35 \%$

Superbugs: $\quad$ Superbugs ${ }^{\circledR}$ micro-organisms from Chemicals Specialties Int., a division of Robinson Research Inc. in Cameron Park, California. Contains:

Bacillus pumilus

Bacillus sp.

Bacillus cereus/thuringiensis

Acinetobacter caloacetincus

Waste Activated charcoal that had been used as a filter medium for

Charcoal: vodka. Currently approximately 1 tonne a week of this charcoal is being disposed of in a sanitary landfill site.

Fly ash: $\quad$ Fly ash from thermal power plants that use pulverized coal was obtained from TransAlta Utilities Corporation, and Alberta Power Ltd. 
Table 5. Rates of amendments for the Devon soil.

\begin{tabular}{|c|c|c|c|c|}
\hline AMENDMENT & $\begin{array}{l}\text { ATRAZINE + } \\
\text { DIURON } \\
\text { (2.18 ppmw a.i.) }\end{array}$ & $\begin{array}{l}\text { ATRAZINE + } \\
\text { DIURON } \\
\text { (1.17 ppmw a.i.) }\end{array}$ & $\begin{array}{l}\text { ATRAZINE }+ \\
\text { DIURON } \\
\text { ( } 0.71 \text { ppmw a.i.) }\end{array}$ & $\begin{array}{l}\text { ATRAZINE }+ \\
\text { DIURON } \\
\text { ( } 0.76 \text { ppmw a.i. })\end{array}$ \\
\hline \multicolumn{5}{|l|}{ CHARCOAL* } \\
\hline $100: 1$ & 218 ppmw & 117 ppmw & $71 \mathrm{ppmw}$ & 76 ppmw \\
\hline $200: 1$ & 436 ppmw & 233 ppmw & 143 ppmw & 153 ppmw \\
\hline
\end{tabular}

MANURE (2:3) $160 \mathrm{~mL}: 240 \mathrm{~mL} \quad 160 \mathrm{~mL}: 240 \mathrm{~mL} \quad 160 \mathrm{~mL}: 240 \mathrm{~mL} \quad 160 \mathrm{~mL}: 240 \mathrm{~mL}$

CHARCOAL plus

MANURE (2:3)

\begin{tabular}{|c|c|c|c|c|}
\hline $200: 1$ & $\begin{array}{l}218 \mathrm{ppmw}+160 \\
\mathrm{~mL} \text { manure }\end{array}$ & $\begin{array}{l}117 \mathrm{ppmw}^{+} \\
160 \mathrm{~mL} \text { manure }\end{array}$ & $\begin{array}{l}71 \mathrm{ppmw}^{+} \\
160 \mathrm{~mL} \text { manure }\end{array}$ & $\begin{array}{l}76 \mathrm{ppmw}+ \\
160 \mathrm{~mL} \text { manure }\end{array}$ \\
\hline $00: 1$ & $\begin{array}{l}436 \text { ppmw }+160 \\
\text { mL manure }\end{array}$ & $\begin{array}{l}233 \mathrm{ppmw}^{+} \\
160 \mathrm{~mL} \text { manure }\end{array}$ & $\begin{array}{l}143 \text { ppmw }+160 \\
\mathrm{~mL} \text { manure }\end{array}$ & $\begin{array}{l}153 \mathrm{ppmw}+ \\
160 \mathrm{~mL} \text { manure }\end{array}$ \\
\hline
\end{tabular}

SAWDUST (2:3) $\quad 160 \mathrm{~mL}: 240 \mathrm{~mL} \quad 160 \mathrm{~mL}: 240 \mathrm{~mL} \quad 160 \mathrm{~mL}: 240 \mathrm{~mL} \quad 160 \mathrm{~mL}: 240 \mathrm{~mL}$

HUMATE 1308 ppmw 308 ppmw 308 ppmw 30

(COMMERCIAL

HUMATE)

HUMATE 2

$5 \mathrm{~g} / 1$

$5 \mathrm{~g} / 1$

$5 \mathrm{~g} / 1$

$5 \mathrm{~g} / 1$

(COMMERCIAL

HUMATE)

SUPERBUGS

n/a

n/a

407 1/ha

407 1/ha

(COMMERCIAL

MICROBES)

Waste Charcoal n/a

n/a

n/a

n/a

Fly ash

n/a

n/a

n/a

n/a

* Refers to ratio of activated charcoal to herbicide active ingredient.

$\mathrm{n} / \mathrm{a}=$ not applicable. Due to insufficient soil volume, the amendments were not tested in this soil. 
Table 6. Rates of amendments for the Vegreville soil.

\begin{tabular}{lrllll}
\hline AMENDMENT & & $\begin{array}{l}\text { BROMACIL } \\
0.8 \mathrm{ppmw}\end{array}$ & $\begin{array}{l}\text { BROMACIL } \\
2.4 \mathrm{ppmw}\end{array}$ & $\begin{array}{l}\text { TEBUTHIURON } \\
0.8 \mathrm{ppmw}\end{array}$ & $\begin{array}{l}\text { TEBUTHIURON } \\
2.4 \mathrm{ppmw}\end{array}$ \\
\hline CHARCOAL $^{*}$ & & & & & \\
& $100: 1$ & $80 \mathrm{ppmw}$ & $240 \mathrm{ppmw}$ & $80 \mathrm{ppmw}$ & $240 \mathrm{ppmw}$ \\
& $200: 1$ & $160 \mathrm{ppmw}$ & $480 \mathrm{ppmw}$ & $160 \mathrm{ppmw}$ & $480 \mathrm{ppmw}$
\end{tabular}

MANURE (2:3)

$160 \mathrm{~mL}: 240 \mathrm{~mL}$

$160 \mathrm{~mL}: 240$

$160 \mathrm{~mL}: 240$

$160 \mathrm{~mL}: 240$

$\mathrm{mL}$

$\mathrm{mL}$

$\mathrm{mL}$

CHARCOAL plus

MANURE (2:3)

$\begin{array}{ll}200: 1 & 80 \mathrm{ppmw}+ \\ & 160 \mathrm{~mL} \text { manure }\end{array}$

$240 \mathrm{ppmw}+\quad 80 \mathrm{ppmw}+$

240 ppmw +

$160 \mathrm{~mL}$ manure $160 \mathrm{~mL}$ manure $160 \mathrm{mLmanure}$

$300: 1$

$160 \mathrm{ppmw}+480 \mathrm{ppmw}+$

160 ppmw +

480 ppmw +

$160 \mathrm{~mL}$ manure

$160 \mathrm{~mL}$ manure

$160 \mathrm{~mL}$ manure

$160 \mathrm{mLmanure}$

SAWDUST (2:3)

$160 \mathrm{~mL}: 240 \mathrm{~mL}$

$160 \mathrm{~mL}: 240$

$160 \mathrm{~mL}: 240$

$160 \mathrm{~mL}: 240$

$\mathrm{mL}$

$\mathrm{mL}$

$\mathrm{mL}$

Humate 1

308 ppmw

308 ppmw

308 ppmw

308 ppmw

(COMMERCIAL

HUMATE)

HUMATE 2

$5 \mathrm{~g} / 1$

$5 \mathrm{~g} / 1$

$5 \mathrm{~g} / 1$

$5 \mathrm{~g} / 1$

(COMMERCIAL

HUMATE)

SUPERBUGS

(COMMERCIAL

MICROBES)

WASte CharcoaL*

(GROUND AND

UNGROUND)

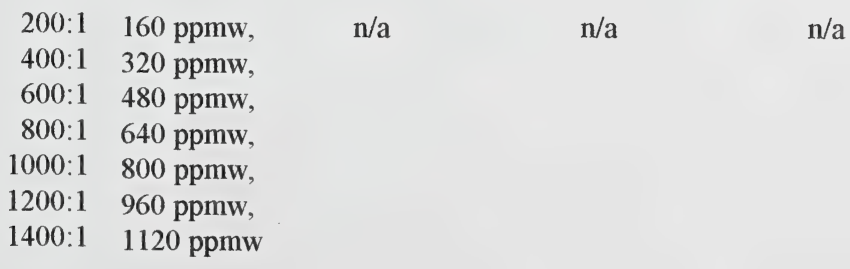

Fly ash

$2,11,22$

$2,11,22$

$\mathrm{n} / \mathrm{a}$

$\mathrm{n} / \mathrm{a}$

(Top ash and bottom tones/ha tones/ha ash - 2 sources)

* Refers to ratio of activated charcoal to herbicide active ingredient.

$\mathrm{n} / \mathrm{a}=$ not applicable. Due to insufficient soil volume, the amendments were not tested in this soil. 

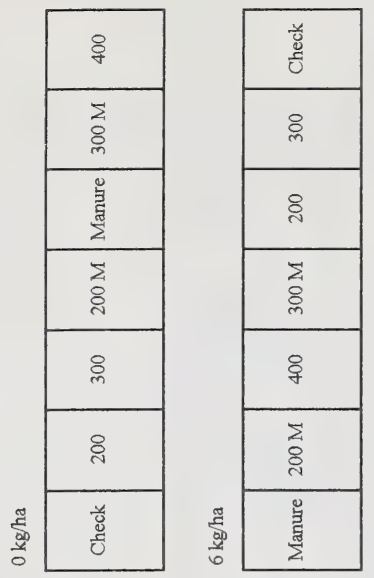

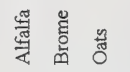
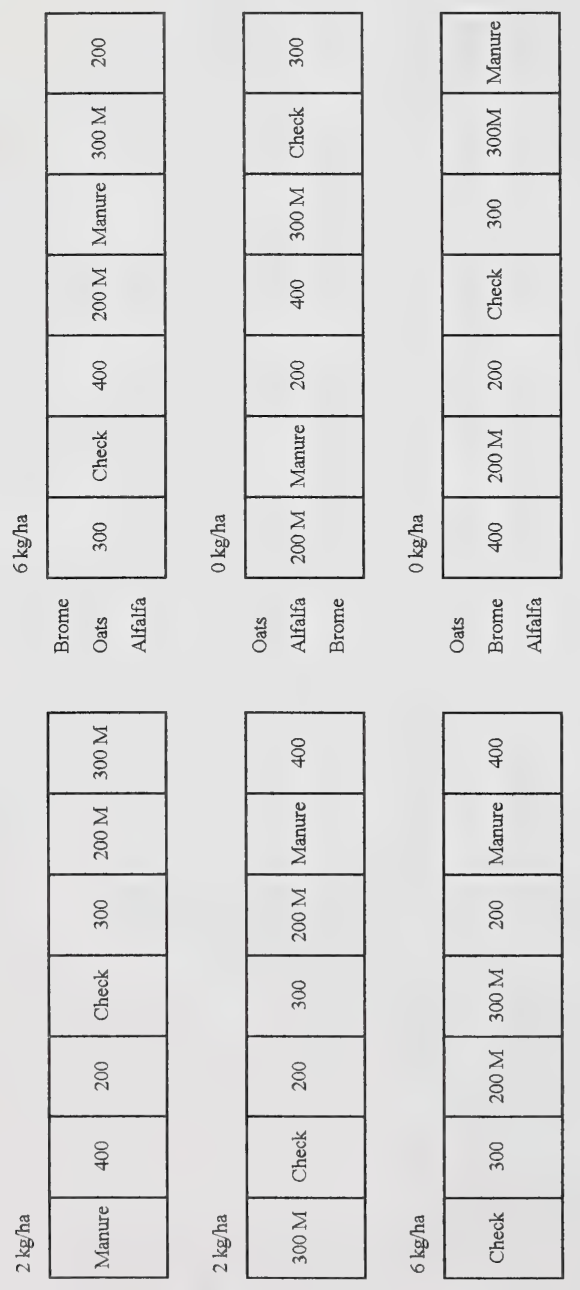

兾量言

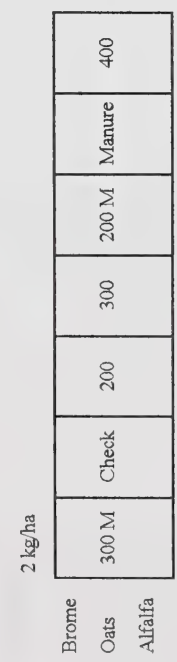

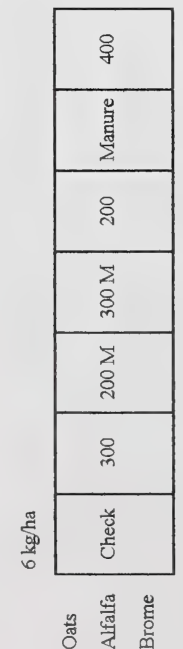

:

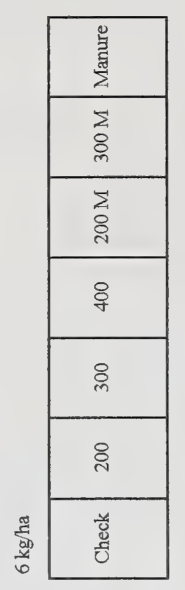

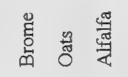
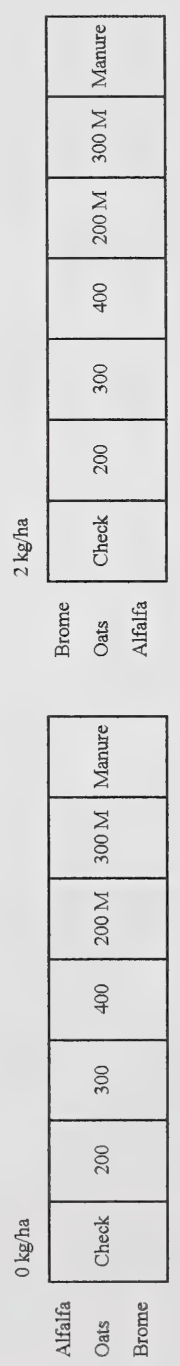

ज苟

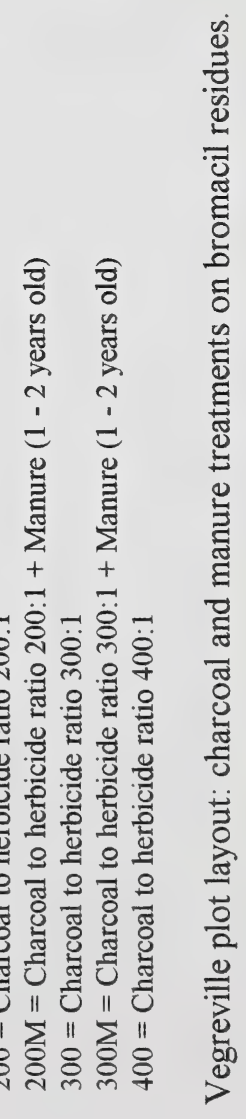

总 


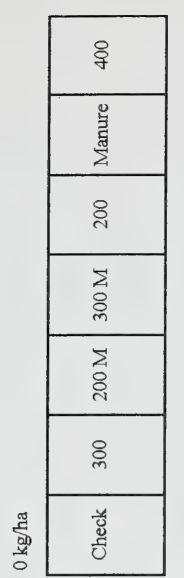

器兽量

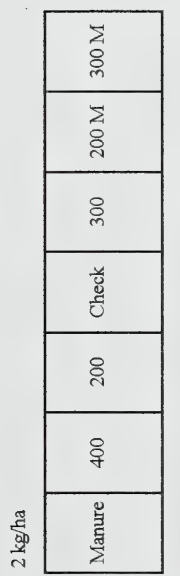

䝮哭意

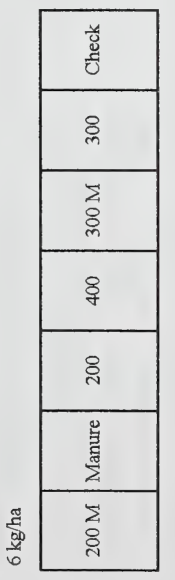

署量曼
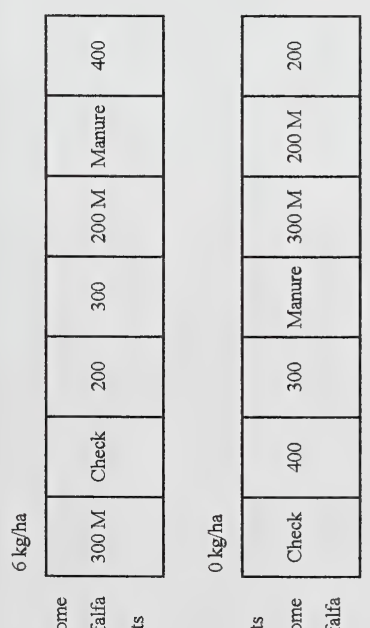

1

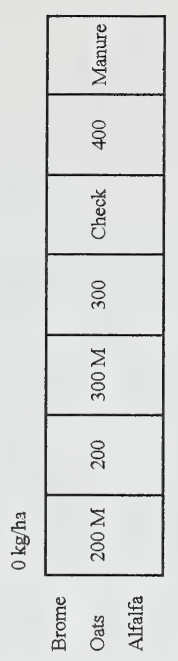

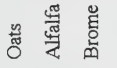
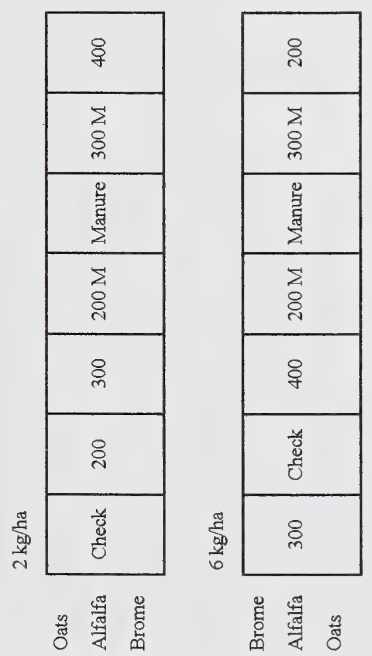

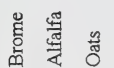

亭

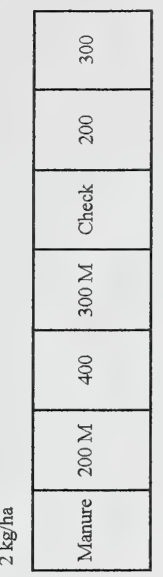

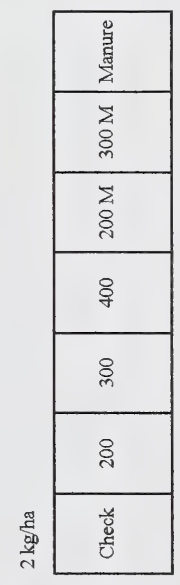

墨总总器
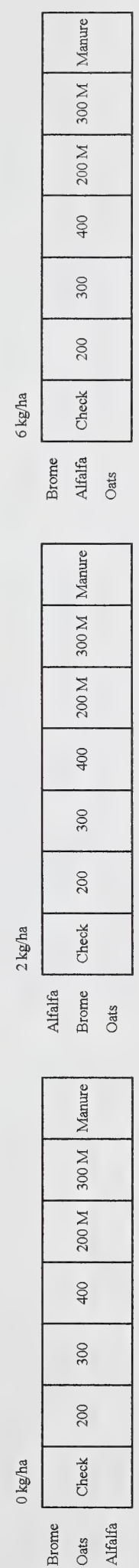

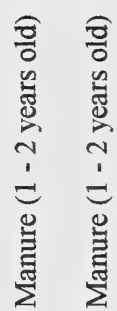

$-\frac{+}{\ddot{8}}-\frac{+}{8}-\quad$ ह

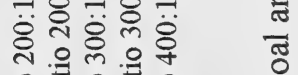

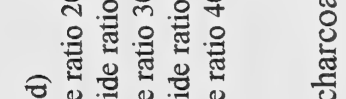

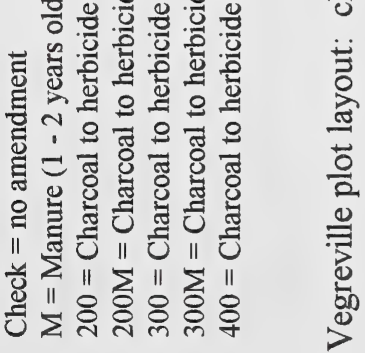

i

苛 

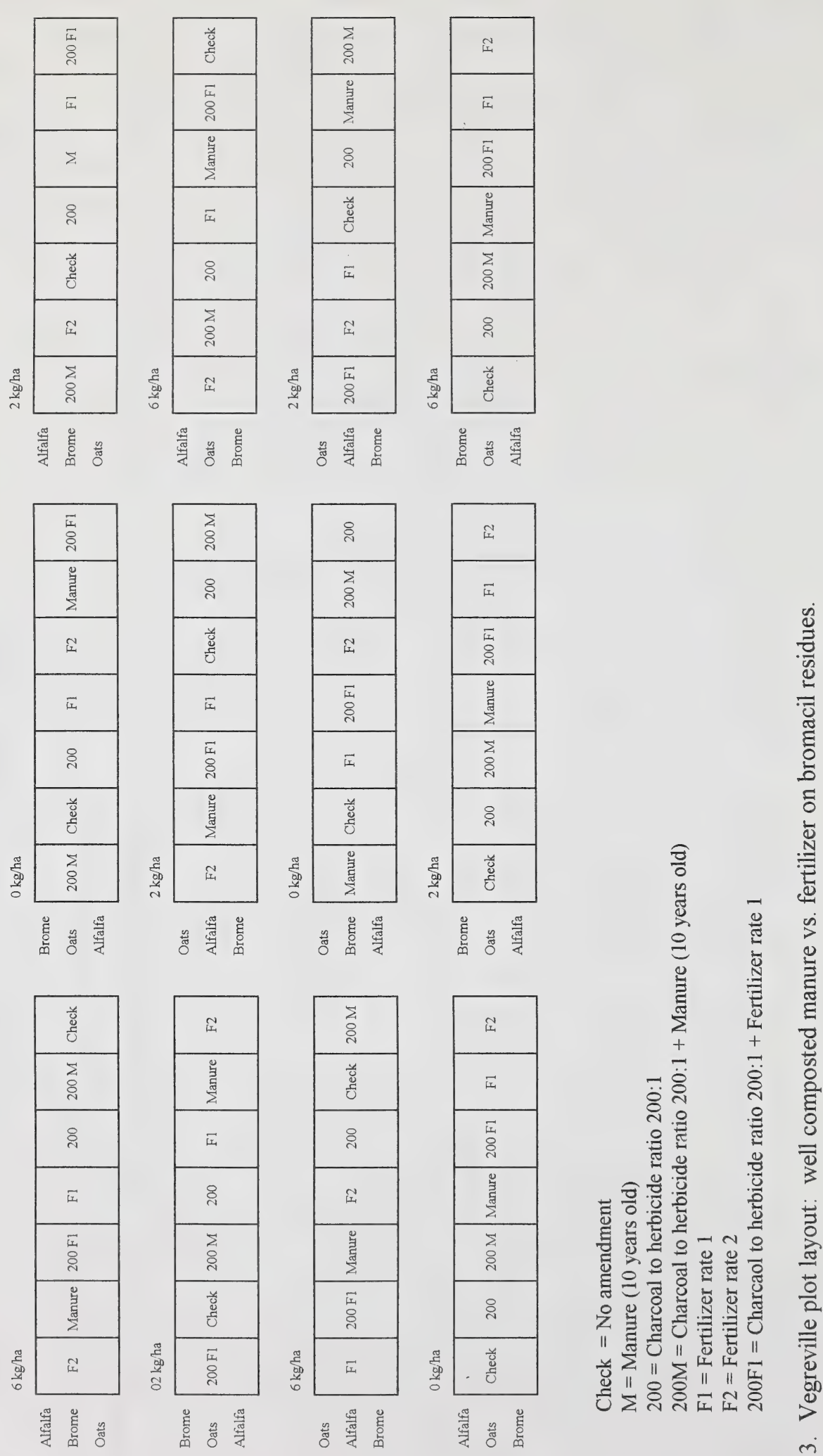


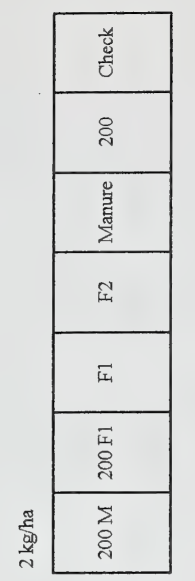

哭量置
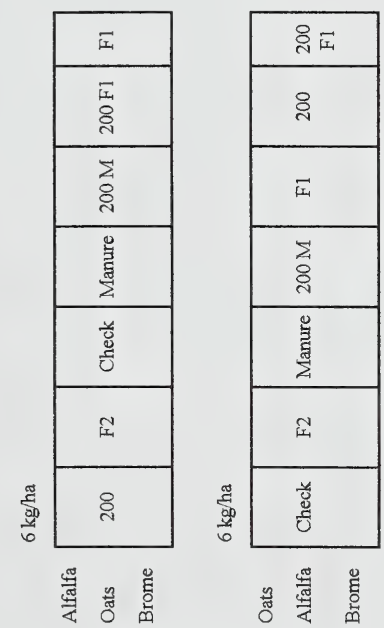

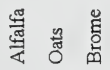
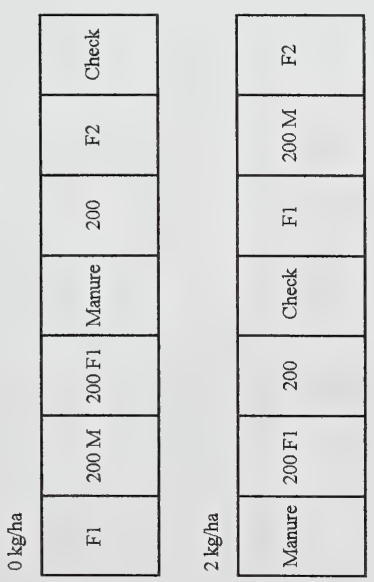

ร 墨量器

咅

离器墨
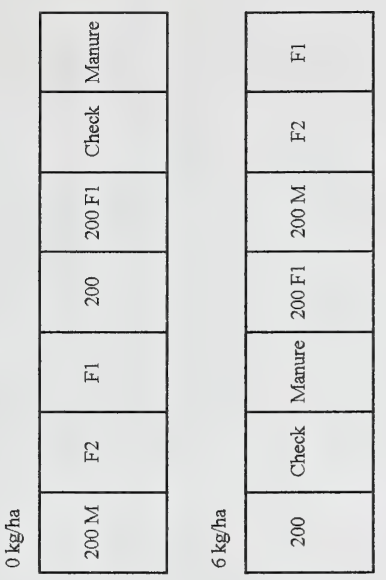

咢变量

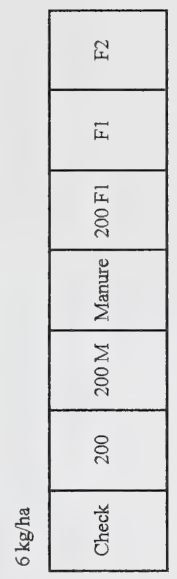

亩圆器

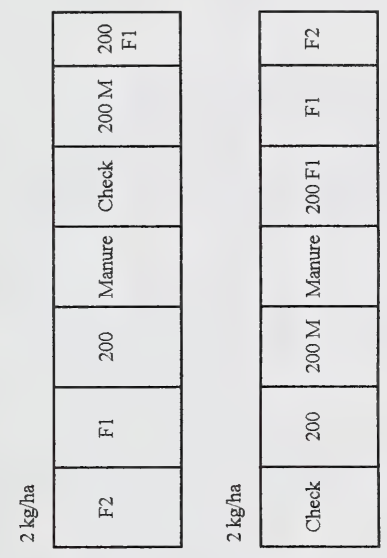

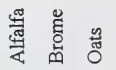

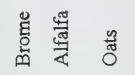

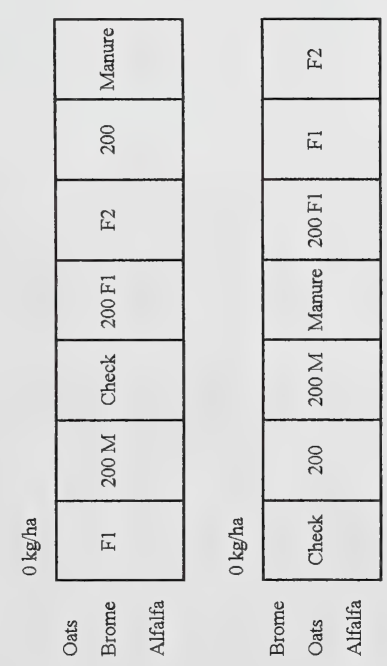

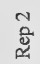




\begin{tabular}{|c|c|c|c|c|c|c|c|c|}
\hline Rep 4 & $\begin{array}{l}\text { Oats } \\
\text { Alfalfa } \\
\text { Brome }\end{array}$ & Check & 200 & 300 & $200 \mathrm{M}$ & Manure & $300 \mathrm{M}$ & 400 \\
\hline $\operatorname{Rep} 3$ & $\begin{array}{l}\text { Brome } \\
\text { Oats } \\
\text { Alfalfa }\end{array}$ & Check & $300 \mathrm{M}$ & $200 \mathrm{M}$ & 300 & 200 & Manure & 400 \\
\hline Rep 2 & $\begin{array}{l}\text { Alfalfa } \\
\text { Brome } \\
\text { Oats }\end{array}$ & $200 \mathrm{M}$ & Manure & 200 & 400 & $300 \mathrm{M}$ & 300 & Check \\
\hline Rep 1 & $\begin{array}{l}\text { Oats } \\
\text { Brome } \\
\text { Alfalfa }\end{array}$ & Check & 200 & 300 & 400 & $200 \mathrm{M}$ & $300 \mathrm{M}$ & Manure \\
\hline
\end{tabular}

Legend: $\quad$ Check $=$ no amendment

$\mathrm{M}=$ Manure ( 5 years old)

$200=$ Charcoal to herbicide ratio 200:1

$200 \mathrm{M}=$ Charcoal to herbicide ratio 200:1 + Manure (5 years old)

$300=$ Charcoal to herbicide ratio $300: 1$

$300 \mathrm{M}=$ Charcoal to herbicide ratio $300: 1+$ Manure $(5$ years old)

$400=$ Charcoal to herbicide ratio $400: 1$

Figure 5. Devon plot layout: charcoal and manure treatments

\begin{tabular}{|c|c|c|c|c|c|c|c|}
\hline Rep 4 & $\begin{array}{l}\text { Alfalfa } \\
\text { Oats } \\
\text { Brome }\end{array}$ & $\begin{array}{l}200 \\
4 \text { Inc. }\end{array}$ & $\begin{array}{l}100 \\
1 \text { Inc. }\end{array}$ & $\begin{array}{l}200 \\
1 \text { Inc. }\end{array}$ & $\begin{array}{l}100 \\
2 \text { Inc. }\end{array}$ & $\begin{array}{l}200 \\
2 \text { Inc. }\end{array}$ & $\begin{array}{r}100 \\
4 \text { Inc. }\end{array}$ \\
\hline $\operatorname{Rep} 3$ & $\begin{array}{l}\text { Oats } \\
\text { Brome } \\
\text { Alfalfa }\end{array}$ & $\begin{array}{c}200 \\
2 \text { Inc. }\end{array}$ & $\begin{array}{l}100 \\
1 \text { Inc. }\end{array}$ & $\begin{array}{l}100 \\
4 \text { Inc. }\end{array}$ & $\begin{array}{r}200 \\
4 \text { Inc. }\end{array}$ & $\begin{array}{c}100 \\
2 \text { Inc. }\end{array}$ & $\begin{array}{l}200 \\
1 \text { Inc. }\end{array}$ \\
\hline Rep & $\begin{array}{l}\text { Alfalfa } \\
\text { Brome } \\
\text { Oats }\end{array}$ & $\begin{array}{l}200 \\
1 \text { Inc. }\end{array}$ & $\begin{array}{l}100 \\
4 \text { Inc. }\end{array}$ & $\begin{array}{l}100 \\
1 \text { Inc. }\end{array}$ & $\begin{array}{r}200 \\
4 \text { Inc. }\end{array}$ & $\begin{array}{c}100 \\
2 \text { Inc. }\end{array}$ & $\begin{array}{r}200 \\
2 \text { Inc. }\end{array}$ \\
\hline Rep 1 & $\begin{array}{l}\text { Brome } \\
\text { Alfalfa } \\
\text { Oats }\end{array}$ & $\begin{array}{l}100 \\
1 \text { Inc. }\end{array}$ & $\begin{array}{l}100 \\
4 \text { Inc. }\end{array}$ & $\begin{array}{l}200 \\
4 \text { Inc. }\end{array}$ & $\begin{array}{l}200 \\
1 \text { Inc. }\end{array}$ & $\begin{array}{l}100 \\
2 \text { Inc. }\end{array}$ & $\begin{array}{r}200 \\
2 \text { Inc. }\end{array}$ \\
\hline
\end{tabular}

Legend: $\quad 100=$ Charcoal to herbicide ratio $100: 1$

$200=$ Charcoal to herbicide ratio 200:1

1 inc. $=1$ incorporation of treatment

2 inc. $=2$ incorporations of treatment

4 inc. $=4$ incorporations of treatment

Figure 6. Devon plot layout: number of incorporations comparison 


\begin{tabular}{|c|c|c|c|c|c|c|c|c|c|c|c|}
\hline $\operatorname{Rep} 4$ & $\begin{array}{l}\text { Oats } \\
\text { Alfalfa } \\
\text { Brome }\end{array}$ & $P$ & Check & M & F1P & FI & $200 \mathrm{M}$ & F2 & $200 \mathrm{~F} 1 \mathrm{P}$ & $200 \mathrm{FI}$ & 200 \\
\hline Rep 3 & $\begin{array}{l}\text { Alfalfa } \\
\text { Brome } \\
\text { Oats }\end{array}$ & M & F2 & $P$ & $200 \mathrm{~F} 1$ & 200 & $200 \mathrm{AP}$ & Check & F1 & F1P & $200 \mathrm{M}$ \\
\hline Rep 2 & $\begin{array}{l}\text { Brome } \\
\text { Alfalfa } \\
\text { Oats }\end{array}$ & $200 \mathrm{~F} 1$ & F1P & F2 & 200 F1P & M & F1 & $200 \mathrm{M}$ & $P$ & Check & 200 \\
\hline Rep 1 & $\begin{array}{l}\text { Oats } \\
\text { Brome } \\
\text { Alfalfa }\end{array}$ & Check & 200 & $200 \mathrm{M}$ & M & $\mathrm{F} 2$ & F1 & F1P & $200 \mathrm{~F} 1$ & 200 F1P & $P$ \\
\hline
\end{tabular}

Legend: $\quad$ Check $=$ no amendment

$\mathrm{P}=$ peat

$M=$ manure (10 years old)

$\mathrm{F} 1=$ fertilizer rate 1

$\mathrm{F} 1 \mathrm{P}=$ fertilizer rate $1+$ peat
F2 = fertilizer rate 2

$200=$ charcoal to herbicide ratio $200: 1$

$200 \mathrm{M}=$ charcoal to herbicide ratio $200: 1+$ manure (10 years old)

$200 \mathrm{~F} 1$ = charcoal to herbicide ratio $200: 1+$ fertilizer rate 1

$200 \mathrm{~F} 1 \mathrm{P}=$ charcoal to herbicide ratio $200: 1+$ fertilizer rate $1+$ peat

Figure 7. Devon plot layout: well composted manure vs. peat and fertilizer treatments

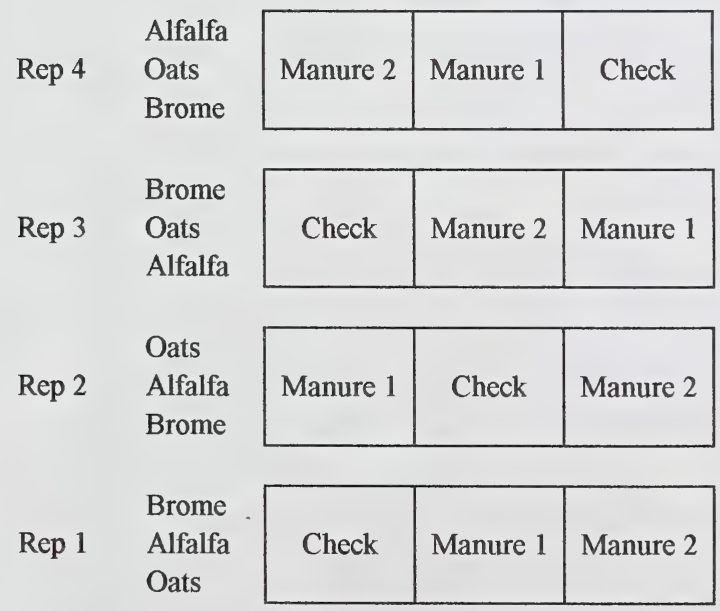

Legend: $\quad$ Check $=$ no amendment

Manure $1 \cong 5$ year old manure

Manure $2 \cong 10$ year old manure

Figure 8. Devon plot layout: Quality of manure comparison 
The activated charcoal was applied as a slurry in water using a tractor mounted spray unit (Figure 9). Table 7 lists the activated charcoal field application rates. The charcoal was mixed as a $10 \%$ suspension in water. Hydrodarco $\mathrm{B}{ }^{\circledR}$ powdered activated carbon 100 plus mesh size was used in all of the field experiments. The charcoal was produced by steam activation of lignite coal. It is a product of American Norit Company, Inc. obtained from Van Waters \& Rogers, Edmonton.

Table 7. Activated charcoal field rates

Ratio of activated charcoal to herbicide active ingredient

Herbicide concentration 200:1 $300: 1$ $400: 1$

Activated Charcoal (kg/ha)

Vegreville (bromacil or tebuthiuron)

$0.8 \mathrm{ppmw}(2 \mathrm{~kg} / \mathrm{ha})$

$2.4 \mathrm{ppmw}(6 \mathrm{~kg} / \mathrm{ha})$

Devon (atrazine and diuron)

$2.18 \mathrm{ppmw}(5.23 \mathrm{~kg} / \mathrm{ha})$

$1.17 \mathrm{ppmw}(2.8 \mathrm{~kg} / \mathrm{ha})$
400

1200

1100

600
600

1800

1600

900
800

2400

2100

1200

A local source of manure was used for the field experiments. Table 8 lists the composition of the manure. The source of manure at Devon was well composted approximately five year old cattle manure. It had no distinguishable straw fibres, and no unpleasant odor. At Vegreville, for the first year of the field experiments, the source was approximately one year old cattle manure not well composted. The manure was obtained from the local cattle operation at the Alberta Environmental Centre. It had a high percent of long straw fibres that were not decomposed. It also had a strong manure odor.

Table 8. Manure composition

\begin{tabular}{llll}
\hline & $\sim 5+$ yrs old & $\sim 1-2$ yrs old & $\sim 10+$ yrs old \\
\hline Organic Matter & $18.4 \%$ & - & - \\
$\mathrm{NH}_{4}$ & $5.8 \mathrm{ppm}$ & $121 \mathrm{ppm}$ & $3.5 \mathrm{ppm}$ \\
$\mathrm{NO}_{3}+\mathrm{NO}_{2}$ & $524 \mathrm{ppm}$ & $201 \mathrm{ppm}$ & $410 \mathrm{ppm}$ \\
Phosphate & $1520 \mathrm{ppm}$ & $815 \mathrm{ppm}$ & $645 \mathrm{ppm}$ \\
Potassium & $6750 \mathrm{ppm}$ & $8380 \mathrm{ppm}$ & $6830 \mathrm{ppm}$ \\
\hline
\end{tabular}




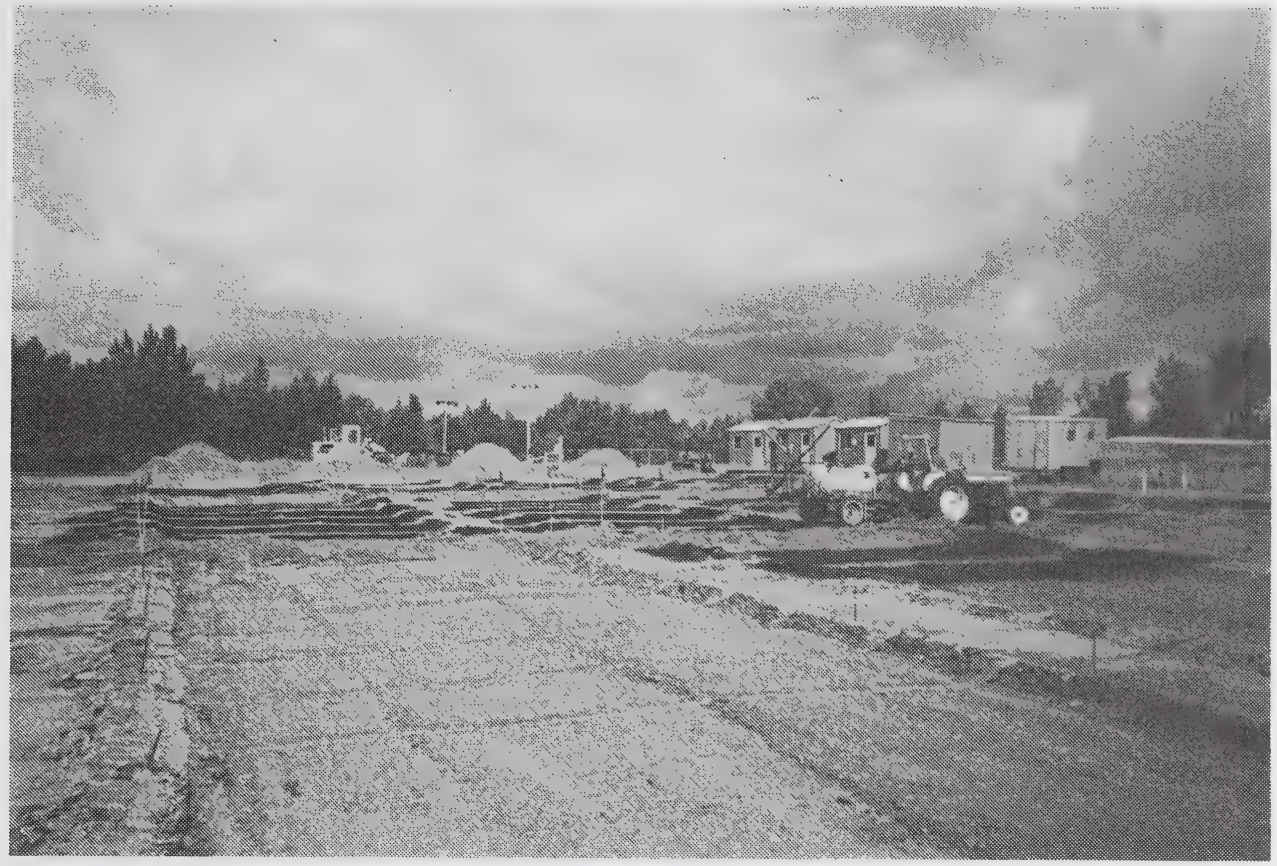

Figure 9. Application of activated charcoal with a tractor mounted spray unit to 15 to $30 \mathrm{~cm}$ soil depth at Devon.

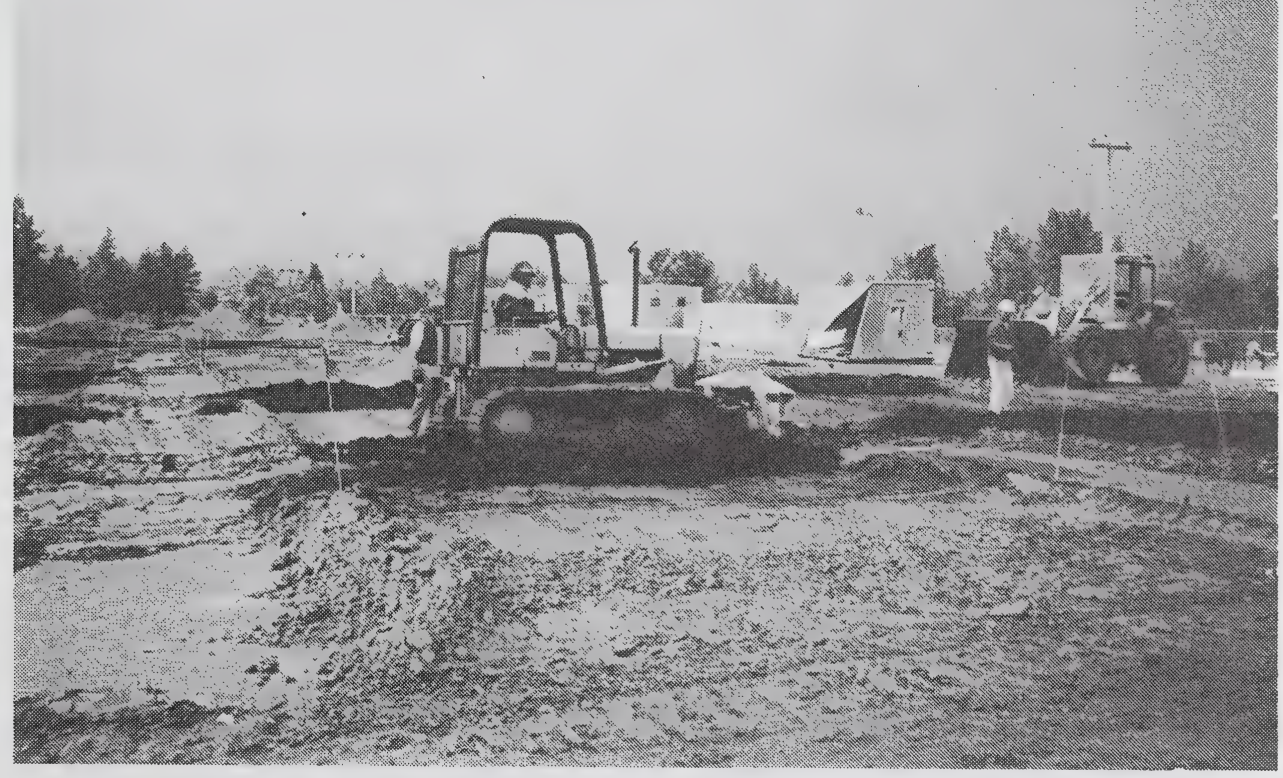

Figure 10. Application of manure using small bulldozer to 15 to $30 \mathrm{~cm}$ depth soil at Devon. 
The manure was to be applied in a 10 to $15 \mathrm{~cm}$ layer on all plots. A manure spreader was used to apply the manure at Vegreville in the first year. The desired accuracy could not be achieved (a 20 to $30 \mathrm{~cm}$ layer of manure resulted $-30 \mathrm{~cm}$ on the tebuthiuron plots, and $20 \mathrm{~cm}$ on the bromacil plots). A small loader was used for the remaining manure applications (Figure 10). Reasonable accuracy could be achieved because the volume of the bucket was calculated, and the appropriated number of buckets was then dumped on each plot, and finally spread. This method resulted in the desired 10 to $15 \mathrm{~cm}$ layer of manure. In the second year at Vegreville, a better manure was obtained and was applied with the loader. It was approximately ten year old composted manure from a dairy farm. The manure had no visible straw fibres and no unpleasant odor. In the second year at Devon (Figure 8), the two manure types were compared.

When peat and fertilizer were introduced into the experiment in the second year, the peat was applied in the same manner as the manure, and the fertilizer was measured in pails and applied by hand. The fertilizer was applied at two rates: (1) approximating the available $\mathrm{N}, \mathrm{P}$, and $\mathrm{K}$ in the local manure; and (2) approximating the available $\mathrm{P}$ and $\mathrm{K}$, and twice the available $\mathrm{N}$. The field rates are presented in Table 9. The peat, a regular Grower's grade sphagnum moss was applied in a 10 to $15 \mathrm{~cm}$ layer.

Table 9. Field rates of fertilizer.

\begin{tabular}{llclc}
\hline Manure description & \multicolumn{2}{l}{ Fertilizer Rate $1(\mathrm{~kg} / \mathrm{ha})$} & \multicolumn{2}{l}{ Fertilizer Rate 2 (kg/ha) } \\
\hline$\sim 5$ years old & $\mathrm{N}$ & 106 & $\mathrm{~N}$ & 212 \\
& $\mathrm{P}_{2} \mathrm{O}_{5}$ & 697 & $\mathrm{P}_{2} \mathrm{O}_{5}$ & 697 \\
& $\mathrm{~K}_{2} \mathrm{O}$ & 1622 & $\mathrm{~K}_{2} \mathrm{O}$ & 1622 \\
$\sim 10$ years old & & & & \\
& $\mathrm{N}$ & 91 & $\mathrm{~N}$ & 182 \\
& $\mathrm{P}_{2} \mathrm{O}_{5}$ & 412 & $\mathrm{P}_{2} \mathrm{O}_{5}$ & 412 \\
& $\mathrm{~K}_{2} \mathrm{O}$ & 2254 & $\mathrm{~K}_{2} \mathrm{O}$ & 2254 \\
\hline
\end{tabular}

All amendments were incorporated using a tractor mounted rotovator (Figure 11). Two passes with the rotovator were conducted for each plot, except for the efficiency of incorporation experiment which had 1, 2 or 4 incorporations.

The manure was to be applied in a 10 to $15 \mathrm{~cm}$ layer on all of the plots. A manure spreader was used to apply the manure at Vegreville in the first year. The desired accuracy could not be achieved (a 20 to $30 \mathrm{~cm}$ layer of manure resulted $--30 \mathrm{~cm}$ on the tebuthiuron plots, and $20 \mathrm{~cm}$ on the bromacil plots). A small loader was used for the remaining manure applications (Figure 10). Reasonable accuracy could be achieved because the volume of the bucket was calculated, and the appropriate number of buckets was then dumped on each plot, and finally spread. This method resulted in the desired 10 to $15 \mathrm{~cm}$ layer of manure. In the second year at 


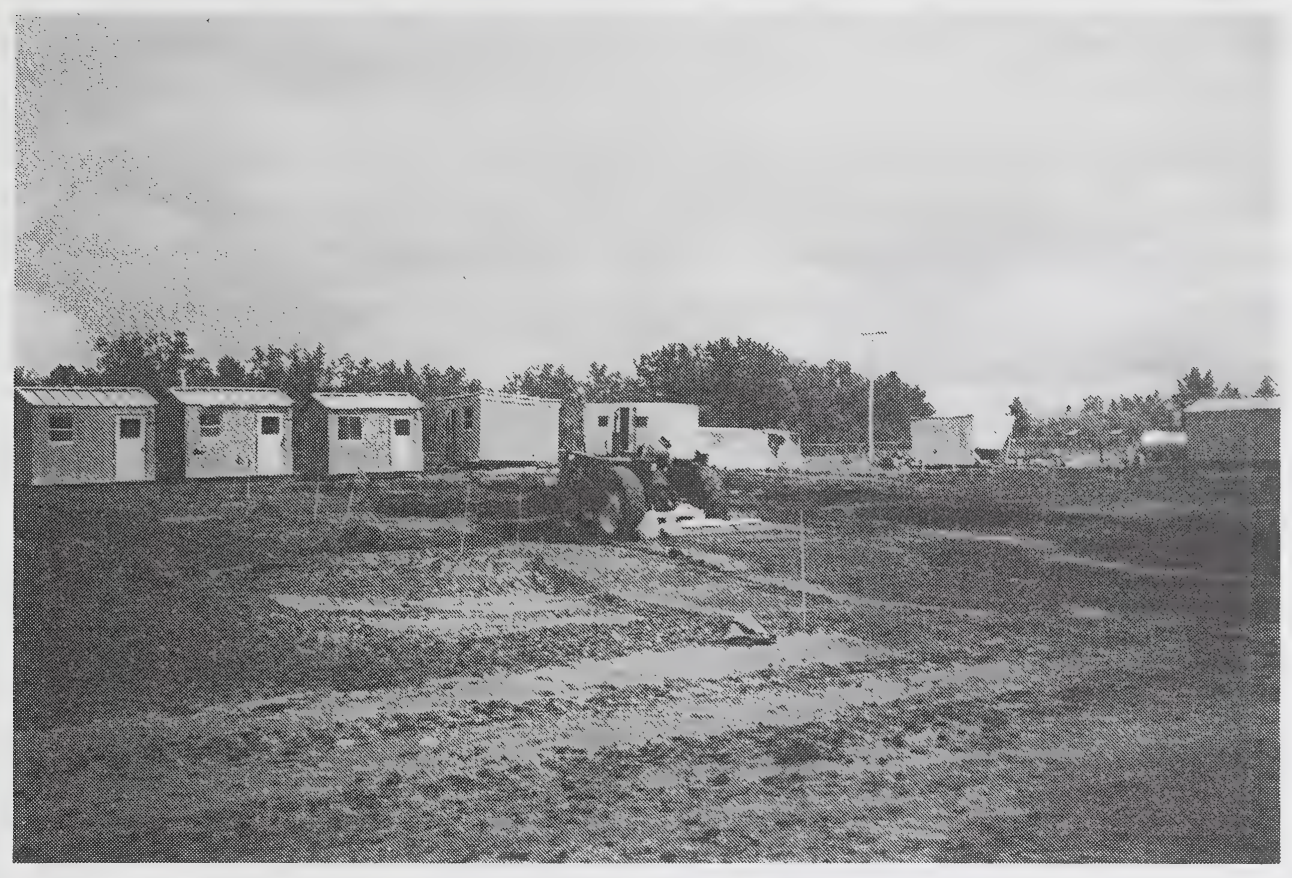

Figure 11. Rotovating the 15 to $30 \mathrm{~cm}$ soil depth at Devon.

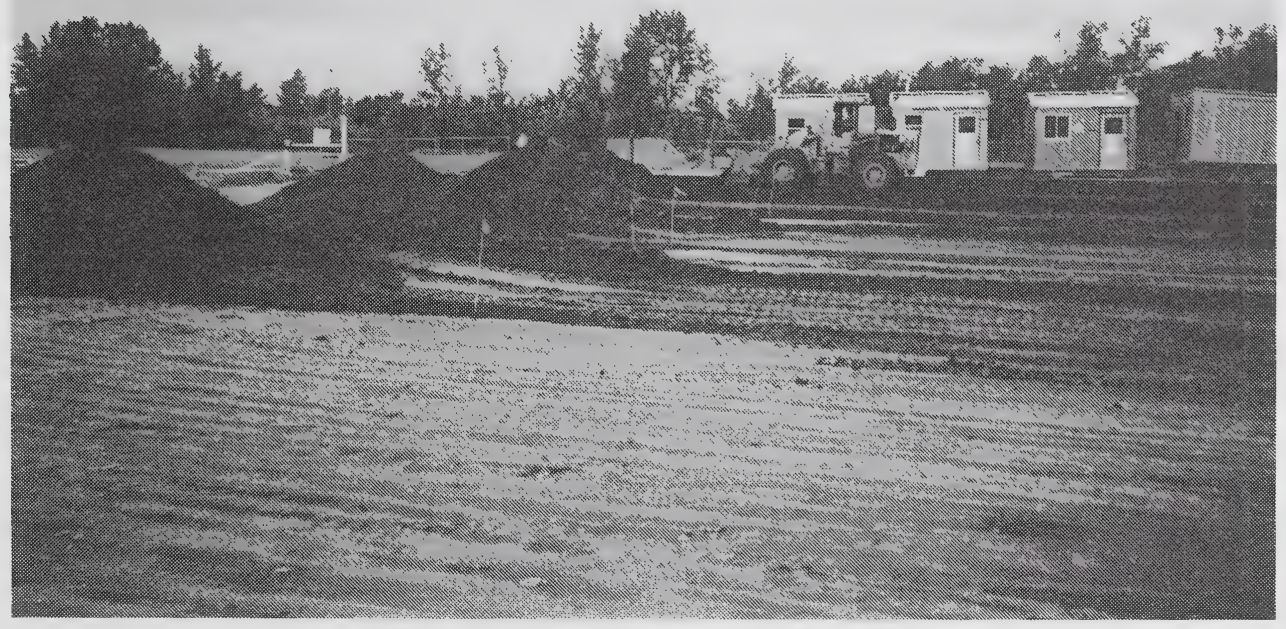

Figure 12. Stripping the top $15 \mathrm{~cm}$ of soil at Devon by block. 
Vegreville, a better manure was obtained and was applied with the loader. It was approximately ten year old.

Each incorporation consisted of two passes with the rotovator. The incorporation depth at Vegreville was approximately $15 \mathrm{~cm}$, as indicated by PVC tube bioassay sampling (Appendix 8.1). Preliminary bioassays using the Devon soil indicated that the depth of herbicide contamination exceeded $120 \mathrm{~cm}$ (Appendix 8.2 shows injury rating after 4 weeks growth). Equipment failure prevented sampling beyond $120 \mathrm{~cm}$.

The amendments were applied to a $30 \mathrm{~cm}$ depth at Devon, because that was thought to be the main rooting depth of two of the crops used in the study: oats and brome. The third crops alfalfa was known to have a particularly deep rooting depth, but it's behavior under these conditions was of interest. In order to apply the amendments to the 15 to $30 \mathrm{~cm}$ layer of the soil, the top $15 \mathrm{~cm}$ was stripped using a loader (Figure 12). The soil was stripped by block (4 blocks for each experiment). After the appropriate rate of amendments was applied and incorporated into the 15 to $30 \mathrm{~cm}$ soil layer, the top 0 to $15 \mathrm{~cm}$ of soil was replaced (Figure 13). The amendments were then applied to the upper layer, and incorporated.

Oats (Avena sativa L.), alfalfa (Medicago sativa L.), and bromegrass (Bromus inermis) were seeded across each plot approximately one week after the amendments were incorporated. These were selected as representative monocotyledon and dicotyledon crops; and as representative annual and perennial crops. Seeding rates were as follows: Calibre oats $80 \mathrm{~kg} / \mathrm{ha}$; Beaver alfalfa $15 \mathrm{~kg} / \mathrm{ha}$; Carlton bromegrass $21 \mathrm{~kg} / \mathrm{ha}$. In the first year fertilizer was applied with the seed at a rate of $5 \mathrm{~kg} / \mathrm{ha} \mathrm{N}, 20 \mathrm{~kg} / \mathrm{ha} \mathrm{P}_{2} \mathrm{O}_{5}$, and $20 \mathrm{~kg} / \mathrm{ha} \mathrm{K} 2_{2} 0$. Oats were reseeded each growing season. Alfalfa and Bromegrass were only seeded in the first growing season. At Vegreville in the first growing season (1991), water was supplied by irrigation as required for normal plant growth, until natural precipitation adequately supplied the crops. In the subsequent years irrigation was not required.

The plants were scored regularly throughout the growing season in the same manner as was used in the growth chamber experiments. Data were collected on dry weight of aerial biomass in a $0.75 \mathrm{sq} . \mathrm{m}$. area from each plot at the end of the growing season for all crops. A mid-summer harvest of the bromegrass and the alfalfa was conducted in the second and subsequent years of an experiment. Data were analyzed using ANOVA and Duncan's Multiple Range test. Soil moisture, EC, and pH data were collected on the plots established in 1992 (Manure vs Peat and Fertilizer). 


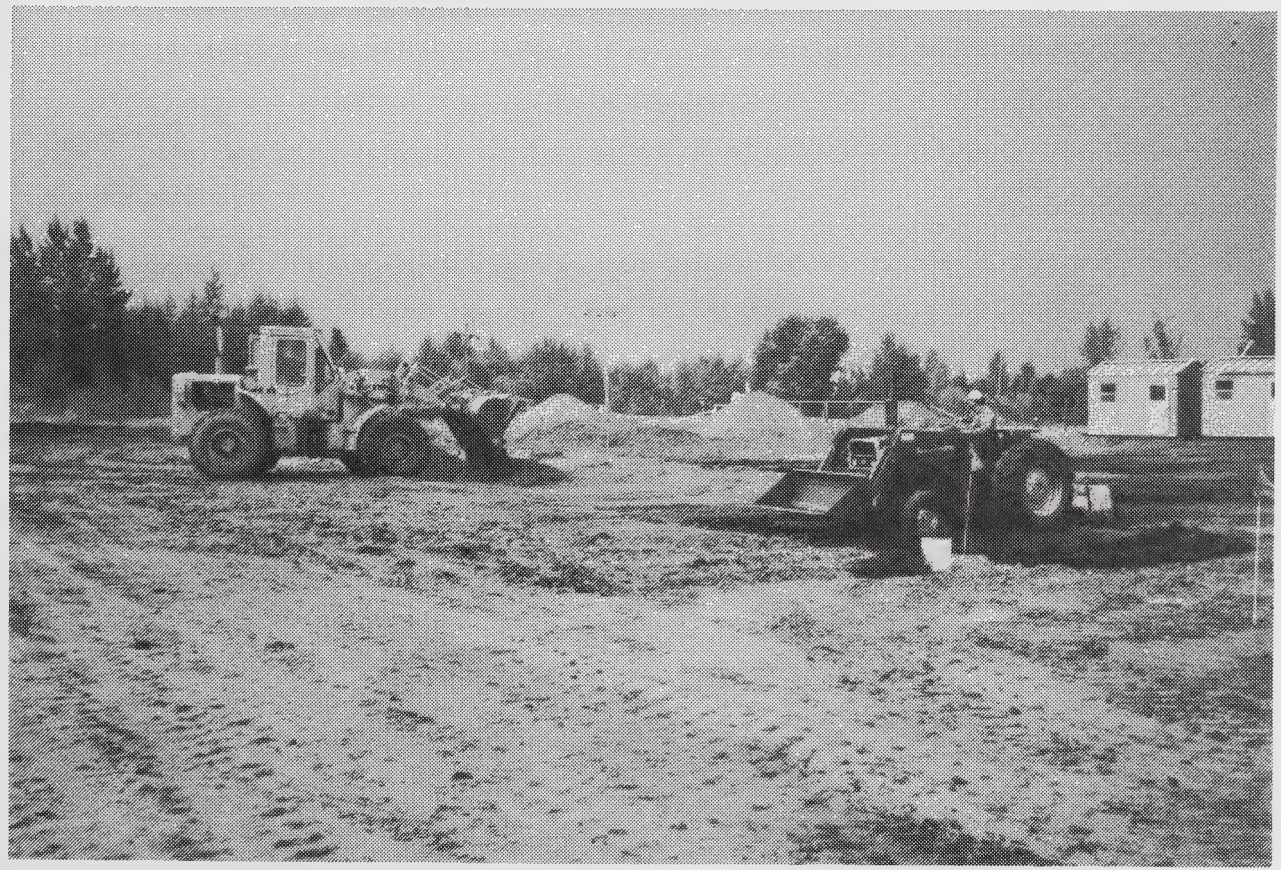

Figure 13. Replacing the top $15 \mathrm{~cm}$ of soil with the loader (right) 


\section{CONTROLLED ENVIRONMENT EXPERIMENTS}

The purpose of the growth chamber bioassay work was to screen the different treatments and choose the most effective treatment to test in the field. None of the treatments prevented herbicide injury to oats at the high bromacil or tebuthiuron rate, in the Vegreville soil. Alfalfa was not protected by any of the treatments at the high bromacil rate, however they were somewhat protected at the high tebuthiuron rate by activated charcoal, and activated charcoal plus manure treatments. Comparison of visual herbicide injury with treatments is shown in Table 10. Figures 14 and 15 show a treatment comparison in mean dry weights for oats grown in 2 and $6 \mathrm{~kg} / \mathrm{ha}$ of tebuthiuron respectively.

At both herbicide rates the treatments of manure alone, and manure plus charcoal produced plants with greater biomass. The plants grown in the charcoal alone were only marginally larger than the controls, particularly at the low herbicide rate. Note that yields are three to ten times higher at the low herbicide rate than the high rate.

The commercial humates, waste activated charcoal, and sawdust did not provide effective protection from herbicide injury. Sawdust applied with no herbicide resulted in severe injury symptoms (Table 10). Fly ash, and waste activated charcoal amendments tested in two separate experiments were found to be ineffective. These treatments resulted in complete death of the crops (data not shown).

Results for controlled environment experiments using Devon soil are presented in Table 11. Charcoal alone (200:1) and charcoal (200:1) plus manure provided satisfactory plant protection from herbicide injury at: (1) 15 to $30 \mathrm{~cm}$ depth (1.17 ppm a.i.); (2) 30 to $45 \mathrm{~cm}$ depth ( $0.71 \mathrm{ppm}$ a.i.); and (3) 45 to $60 \mathrm{~cm}$ depth ( $0.76 \mathrm{ppm}$ a.i.). None of these treatments were effective at the 0 to $15 \mathrm{~cm}$ depth $(2.18 \mathrm{ppm})$. The plants grown in pots that received the charcoal plus a manure had greater biomass than the plants grown in pots receiving charcoal alone (data not shown). It was speculated that this was due to increased nutrients and water holding capacity provided by the manure. The remaining treatments did not provide effective protection from herbicide injury.

From chemical analysis the 30 to $45 \mathrm{~cm}$ depth, and the 45 to $60 \mathrm{~cm}$ depth essentially had the same level of herbicide contamination $(0.71 \mathrm{ppm} v s .0 .76 \mathrm{ppm})$, however in the bioassay study they behaved very differently. The 45 to $60 \mathrm{~cm}$ depth did not have the same degree of injury as the 30 to $45 \mathrm{~cm}$ depth. The herbicide residue levels in the field can be heterogeneous, 
Table 10. Vegreville Soil Bioassay Study - Injury Rating After Four Weeks Growth

\begin{tabular}{|c|c|c|c|c|c|c|}
\hline \multirow[b]{2}{*}{$\mathrm{kg} / \mathrm{ha}$} & \multirow[b]{2}{*}{0} & \multicolumn{2}{|c|}{ Bromacil } & \multirow[b]{2}{*}{0} & \multicolumn{2}{|c|}{ Tebuthiuron } \\
\hline & & 2 & 6 & & 2 & 6 \\
\hline \multicolumn{7}{|l|}{ Crop : Oats } \\
\hline No Amendment & 9 & 2 & 0 & 9 & 5 & 0 \\
\hline Charcoal (100:1) & 9 & 6 & 0 & 8 & 6 & 0 \\
\hline Charcoal (200:1) & 9 & 6 & 0 & 9 & 7 & 0 \\
\hline Manure $(10 \mathrm{~cm})$ & 9 & 9 & 0 & 9 & 7 & 1 \\
\hline \multicolumn{7}{|l|}{ Manure $(10 \mathrm{~cm})+$} \\
\hline Charcoal (100:1) & 9 & 9 & 0 & 9 & 9 & 0 \\
\hline \multicolumn{7}{|l|}{ Manure $(10 \mathrm{~cm})+$} \\
\hline Charcoal $(200: 1)$ & 9 & 9 & 0 & 9 & 9 & 1 \\
\hline Sawdust $(10 \mathrm{~cm})$ & 5 & 4 & 0 & 5 & 4 & 1 \\
\hline Humate 1 & 9 & 2 & 0 & 9 & 5 & 0 \\
\hline Humate 2 & 9 & 1 & 0 & 9 & 4 & 0 \\
\hline Superbugs & 9 & 1 & 0 & 9 & 6 & 0 \\
\hline \multicolumn{7}{|l|}{ Crop: Alfalfa } \\
\hline No Amendment & 9 & 0 & 0 & 9 & 6 & 0 \\
\hline Charcoal (100:1) & 8 & 5 & 0 & 8 & 7 & 3 \\
\hline Charcoal (200:1) & 9 & 6 & 0 & 7 & 7 & 6 \\
\hline Manure $(10 \mathrm{~cm})$ & 8 & 5 & 0 & 8 & 7 & 2 \\
\hline \multicolumn{7}{|l|}{ Manure $(10 \mathrm{~cm})+$} \\
\hline Charcoal (100:1) & 9 & 4 & 0 & 9 & 9 & 1 \\
\hline \multicolumn{7}{|l|}{ Manure $(10 \mathrm{~cm})+$} \\
\hline Charcoal (200:1) & 9 & 5 & 0 & 7 & 7 & 4 \\
\hline Sawdust $(10 \mathrm{~cm})$ & 5 & 3 & 0 & 5 & 4 & 1 \\
\hline Humate 1 & 9 & 3 & 0 & 9 & 7 & 0 \\
\hline Humate 2 & 9 & 0 & 0 & 9 & 8 & 0 \\
\hline Superbugs & 9 & 4 & 0 & 9 & 8 & 0 \\
\hline
\end{tabular}

Note: Plants were visually scored for herbicide injury on a 0 to 9 scale where $0=$ complete kill, $9=$ No visible effect. Refer to Appendix $\mathbf{8 . 2}$ for a more complete rating scale for crop tolerance. 100:1, 200:1 - Refers to ratio of activated charcoal to herbicide active ingredient. 

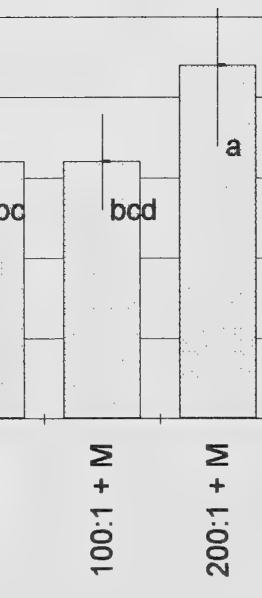

\section{Treatments}

Figure 14. Bioassay experiment using the Vegreville soil with tebuthiuron residues at $2.4 \mathrm{ppm}$. Data shown as mean dry weight of oats in gm/plant $(n=4)$. The treatments were found to be significantly different using Duncans Multiple Range $(P=0.0001)$. Bars with the same letter are not significantly different. Lines represent standard deviation. All of the plants grown in the pots with these treatments had herbicide injury. 

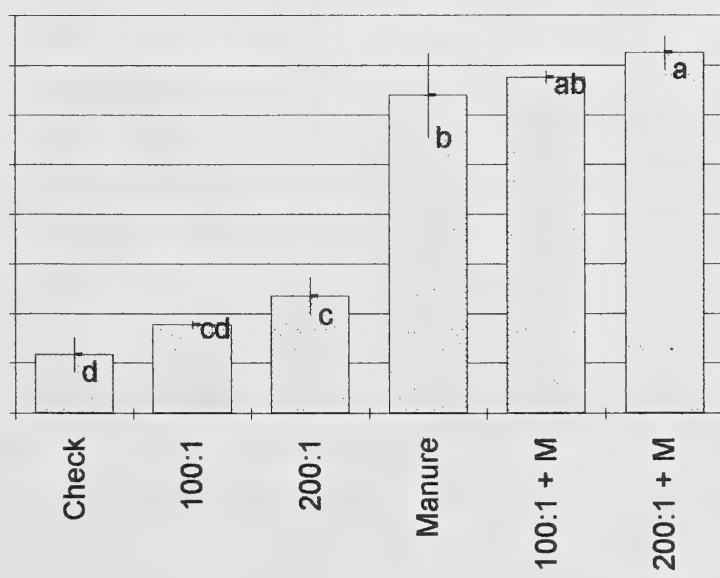

Treatments

Figure 15. Bioassay experiment using the Vegreville soil with tebuthiuron residues at $0.8 \mathrm{ppm}$. Data shown as mean dry weight of oats in $\mathrm{gm} / \mathrm{plant}(\mathrm{n}=4)$. The treatments were found to be significantly different using Duncan's Multiple Range $(P=0.0001)$. Bars with the same letter are not significantly different. Lines represent standard deviation. All of the plants grown in the pots with these treatments had herbicide injury. 
Table 11. Devon Soil Bioassay Study - Injury Rating After Four Weeks Growth

Depth in $\mathrm{cm}$

$0-15$

$15-30$

$30-45$

$45-60$

\begin{tabular}{|c|c|c|c|c|}
\hline Crop : Oats & & & & \\
\hline No Amendment & 0 & 0 & 0 & 5 \\
\hline Charcoal (100:1) & 0 & 1 & 2 & 8 \\
\hline Charcoal (200:1) & 3 & 7 & 9 & 9 \\
\hline Manure $(10 \mathrm{~cm})$ & 0 & 0 & 1 & 6 \\
\hline Manure $(10 \mathrm{~cm})+$ & & & & \\
\hline Charcoal (100:1) & 0 & 2 & 5 & 8 \\
\hline Manure $(10 \mathrm{~cm})+$ & & & & \\
\hline Charcoal (200:1) & 3 & 8 & 9 & 9 \\
\hline Sawdust $(10 \mathrm{~cm})$ & 0 & 0 & 2 & 9 \\
\hline Humate 1 & 0 & 0 & 0 & 4 \\
\hline Humate 2 & 0 & 0 & 0 & 5 \\
\hline Superbugs & N/A & N/A & 0 & 0 \\
\hline Crop Alfalfa & & & & \\
\hline No Amendment & 0 & 0 & 0 & 1 \\
\hline Charcoal (100:1) & 0 & 1 & 1 & 8 \\
\hline Charcoal (200:1) & 1 & 8 & 6 & 9 \\
\hline Manure $(10 \mathrm{~cm})$ & 0 & 1 & 5 & 6 \\
\hline Manure $(10 \mathrm{~cm})+$ & & & & \\
\hline Charcoal (100:1) & 0 & 6 & 5 & 8 \\
\hline Manure $(10 \mathrm{~cm})+$ & & & & \\
\hline Charcoal (200:1) & 1 & 8 & 8 & 8 \\
\hline Sawdust $(10 \mathrm{~cm})$ & 0 & 0 & 0 & 7 \\
\hline Humate 1 & 0 & 0 & 0 & 1 \\
\hline Humate 2 & 0 & 0 & 0 & 1 \\
\hline Superbugs & N/A & N/A & N/A & N/A \\
\hline
\end{tabular}

Note: Plants were visually scored for herbicide injury on a 0 to 9 scale where $0=$ complete kill, $9=$ No visible effect. Refer to Appendix 2 for a more complete rating scale for crop tolerance.

100:1, 200:1 - Refers to ratio of activated charcoal to herbicide active ingredient.

$\mathrm{n} / \mathrm{a}$ - not applicable. Insufficient soil volume did not allow the indicated treatments to be tested on the soil. 
and the soil used in the bioassays may not have been thoroughly mixed, or there may have been a problem with the chemical analysis.

On the basis of the growth chamber work charcoal alone, charcoal plus manure, and manure alone treatments were chosen for the field experiments. However, the ratios of activated charcoal to herbicide were increased to 200:1, 300:1 and 400:1 because the growth chamber study indicated that the 100:1 ratio was too low to be effective.

\subsection{FIELD EXPERIMENTS}

The results for the field experiments at Vegreville and Devon sites over the three year period are presented in Figures 16 to 34.

\subsection{1 $\quad$ Activated Charcoal}

At Vegreville and Devon, all of the activated charcoal treatments prevented herbicide injury to oats, alfalfa, and bromegrass in 1991, 1992, and 1993. No significant difference in crop growth was found at activated charcoal to herbicide ratios of 200:1, 300:1 and 400:1 under field conditions (Figures 16 to 26).

\subsubsection{Manure Treatments}

\subsubsection{Vegreville}

At Vegreville in 1991, manure, and manure plus charcoal were not effective in preventing herbicide injury to crops. The plants grown in bromacil and tebuthiuron residues with manure treatments were chlorotic or dead from herbicide injury (Figure 27). Due to the success of the 5 year old manure at the Devon site (as discussed in the next section), it was surmised that the results at Vegreville may have been affected by the relatively fresh manure. It was approximately one year old cattle manure (refer to Table 8 for manure composition). The manure application rate may also have been too high. It was to have been applied in a $10 \mathrm{~cm}$ layer, but due to application error 20 to $30 \mathrm{~cm}$ was applied. The method of application was altered for the following experiments.

By the second year (1992), the manure had a chance to decompose in the soil. Less straw, and smaller fibres of straw were visible in the manure. The crops grown on manure plus charcoal treatments showed no herbicide injury, and good growth was established (Figure 19 and 20). The treatment of manure alone remained ineffective. There was little growth on the plots 


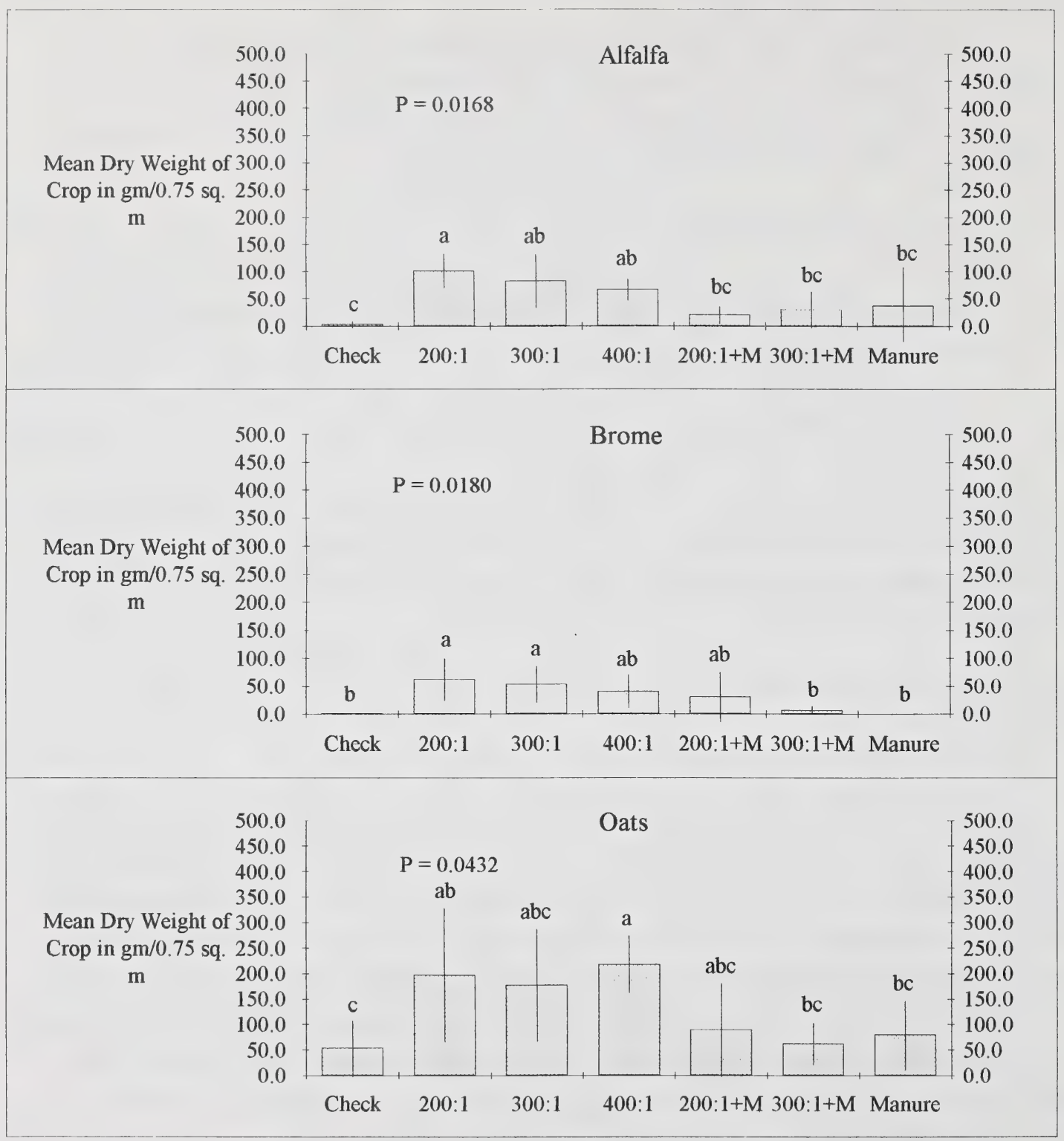

Figure 16. Treatment comparison on the Vegreville soil with initial bromacil residues of $2 \mathrm{~kg} / \mathrm{ha}$ fall 1991 harvest. Data shown as mean dry weight of alfalfa, brome, and oats in gm/0.75 sq.m. $(n=4)$. Bars with the same letter are not significantly different according to Duncan's Multiple Range Test. Lines represent standard deviation. 


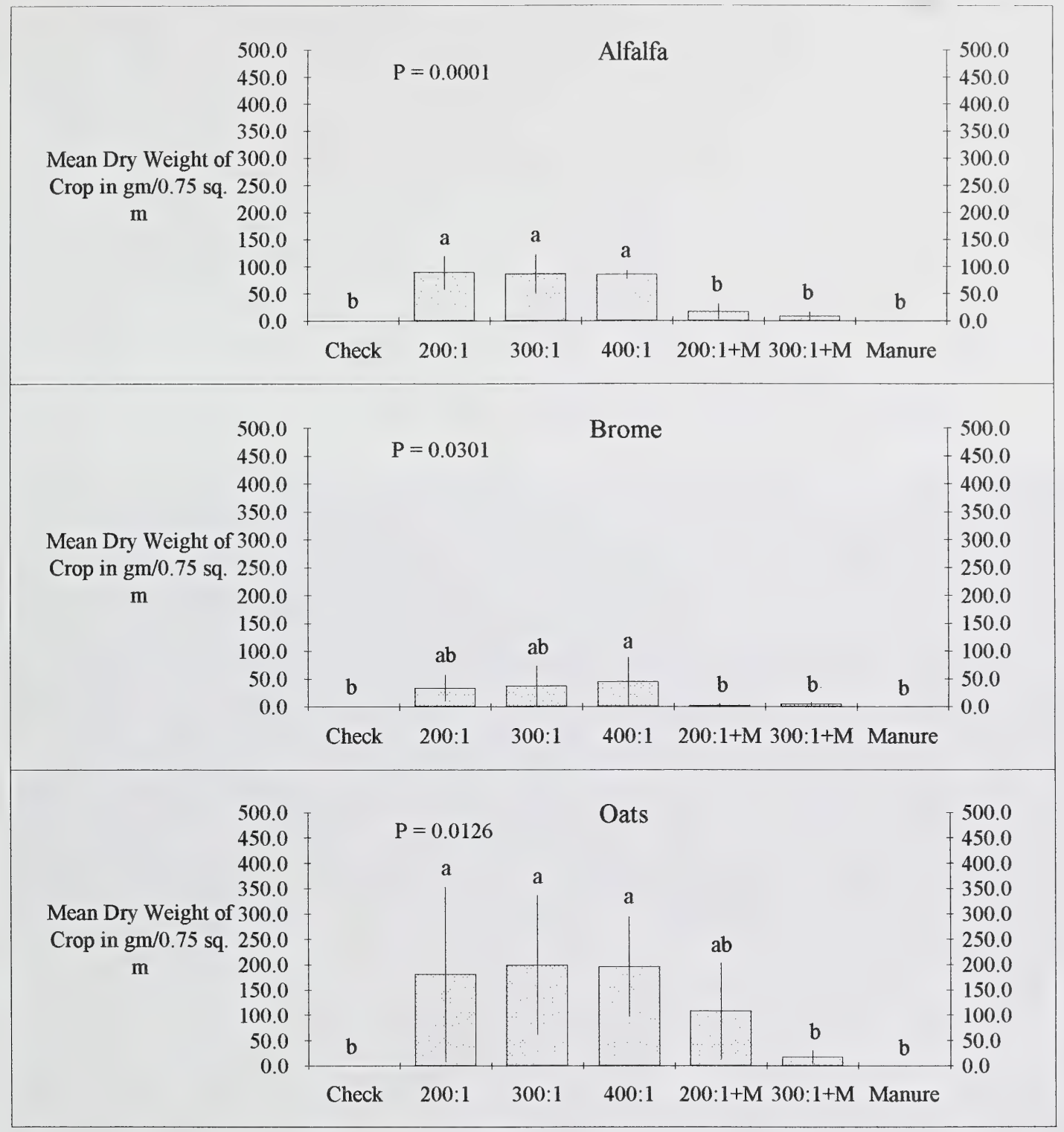

Figure 17. Treatment comparison on the Vegreville soil with initial bromacil residues of $6 \mathrm{~kg} / \mathrm{ha}$ fall 1991 harvest. Data shown as mean dry weight of alfalfa, brome, and oats in gm/ $0.75 \mathrm{sq} . \mathrm{m}$. $(\mathrm{n}=4)$. Bars with the same letter are not significantly different according to Duncan's Multiple Range Test. Lines represent standard deviation. 


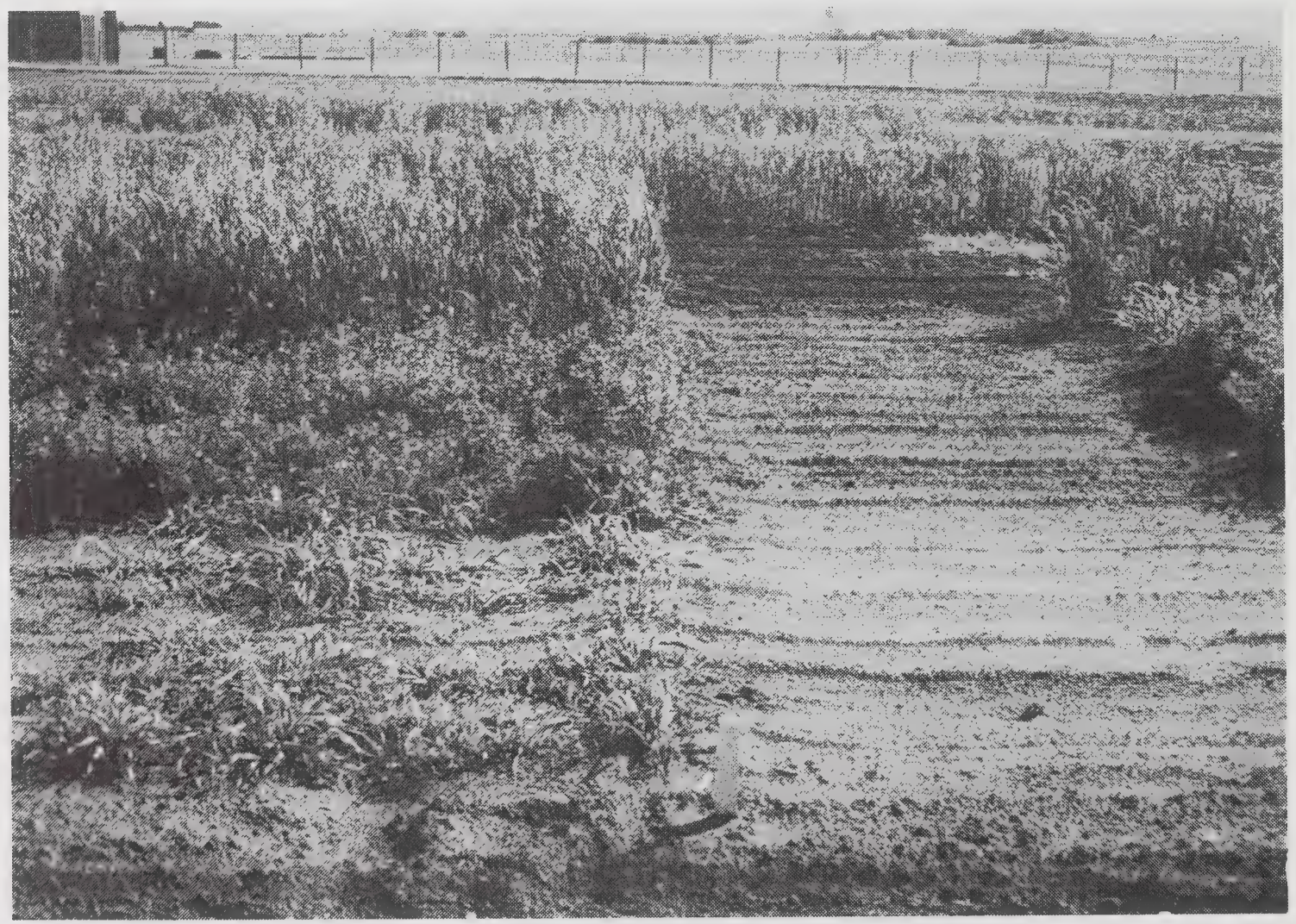

Figure 18. Photograph of bromegrass (foreground), alfalfa (middle), and oats (background) grown in soil treated with bromacil at $6 \mathrm{~kg} / \mathrm{ha}$ at Vegreville in the first growing season. Left plot: ratio of activated charcoal to herbicide active ingredient $200: 1$ (1200 kg/ha of activated charcoal: $6 \mathrm{~kg} / \mathrm{ha}$ of bromacil); Right plot no amendment. 


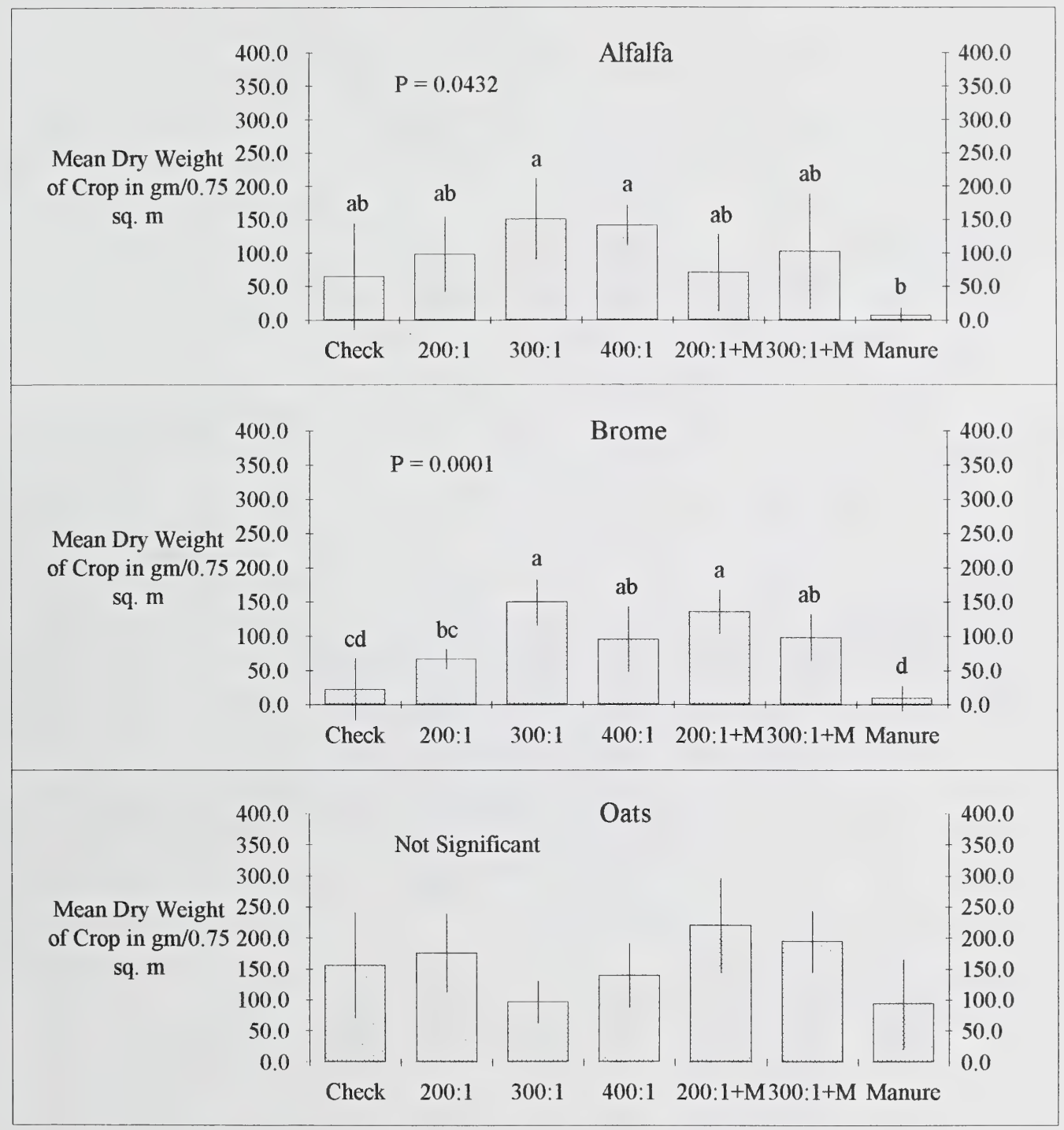

Figure 19. Treatment comparison on the Vegreville soil with initial bromacil residues of $2 \mathrm{~kg} / \mathrm{ha}$ 1992 fall harvest. Data shown as mean dry weight of alfalfa, brome and oats in gm/0.75 sq.m. $(n=4)$. The treatments were not significantly different using Duncan's Multiple Range Test Lines represent standard deviation. 


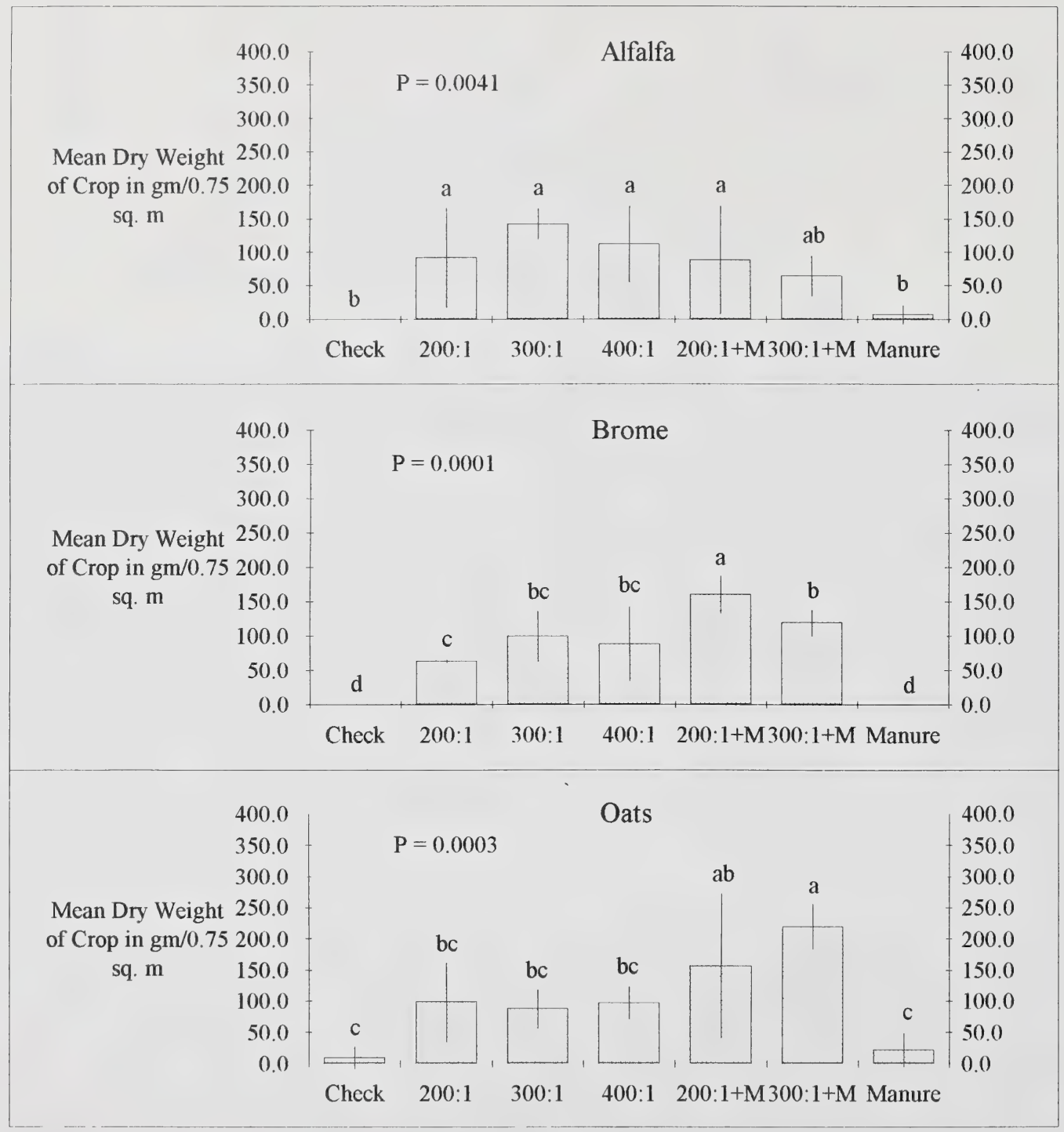

Figure 20. Treatment comparison on the Vegreville soil with initial bromacil residues of $6 \mathrm{~kg} / \mathrm{ha}$ fall 1992 harvest. Data shown as mean dry weight of alfalfa, brome, and oats in gm/0.75 sq.m. $(n=4)$. Bars with the same letter are not significantly different according to Duncan's Multiple Range Test. Lines represent standard deviation. 


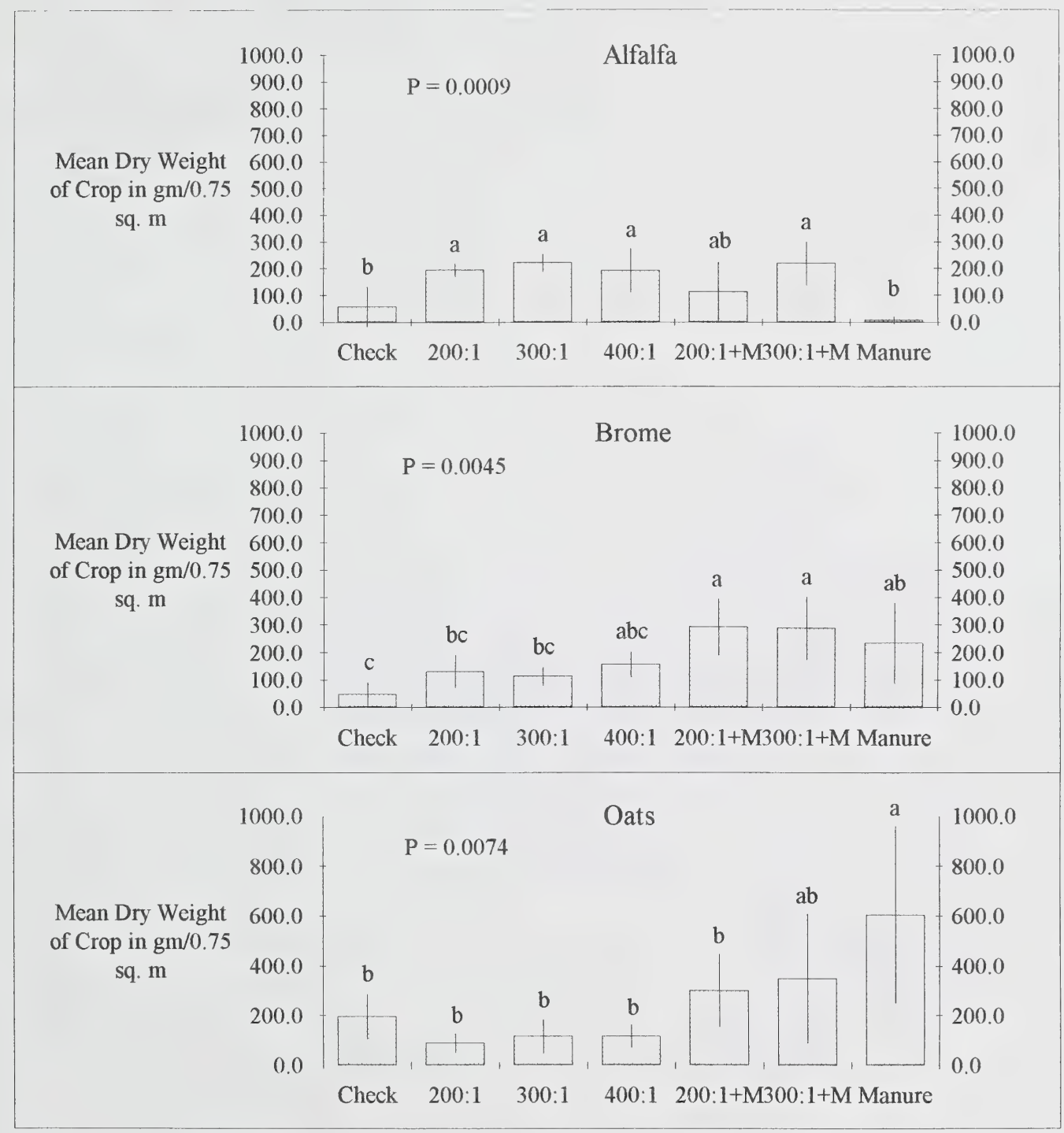

Figure 21. Treatment comparison on the Vegreville soil with initial bromacil residues of $2 \mathrm{~kg} / \mathrm{ha}$ fall 1993 harvest. Data shown as mean dry weight of alfalfa, brome and oats in gm/0.75 sq.m. $(n=4)$. Bars with the same letter are not significantly different according to Duncan's Multiple Range Test. Lines represent standard deviation. 


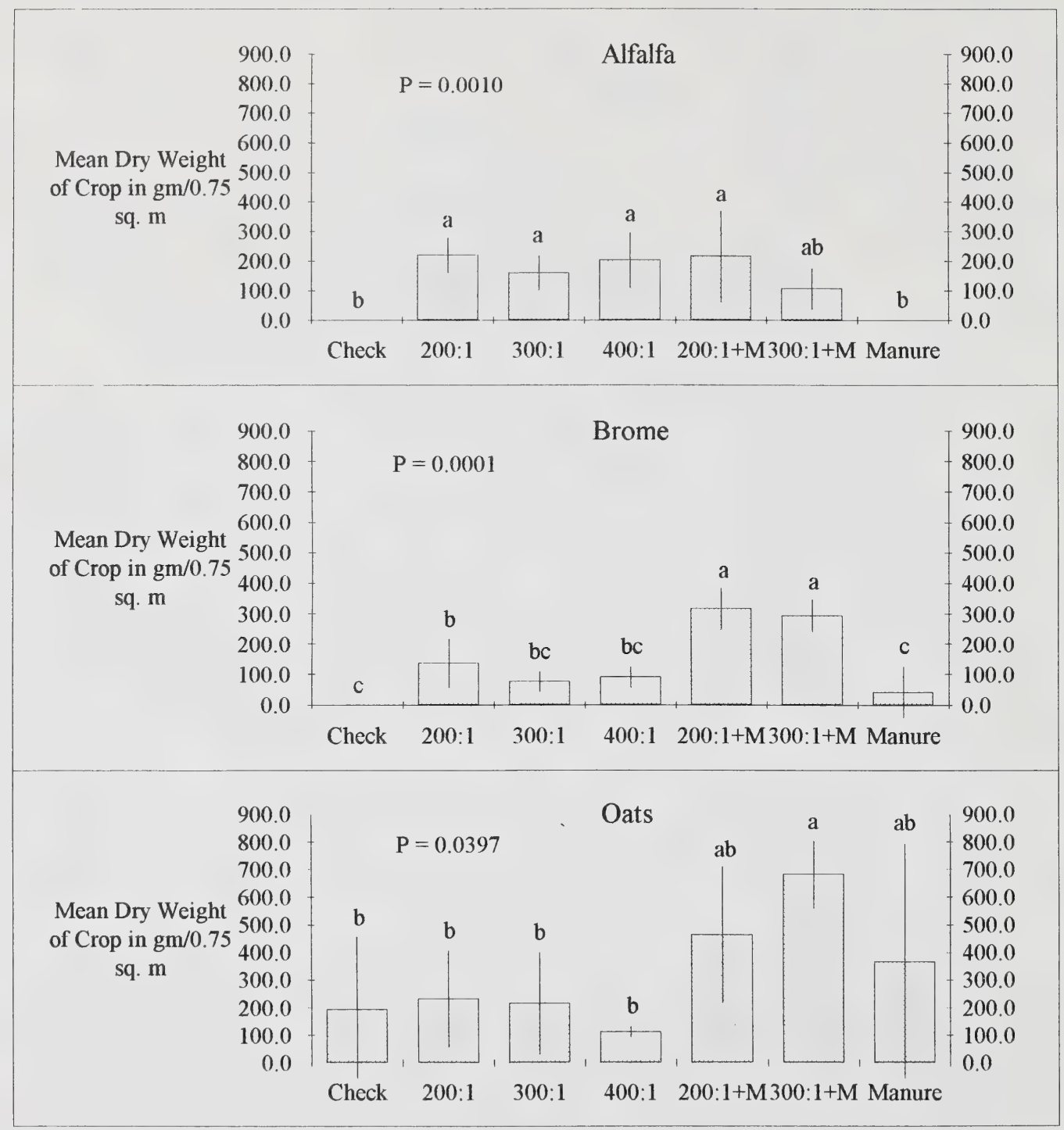

Figure 22. Treatment comparison on the Vegreville soil with initial bromacil residues of $6 \mathrm{~kg} / \mathrm{ha}$ fall 1993 harvest. Data shown as mean dry weight of alfalfa, brome and oats in gm/0.75 sq.m. $(n=4)$. Bars with the same letter are not significantly different according to Duncan's Multiple Range Test. Lines represent standard deviation. 


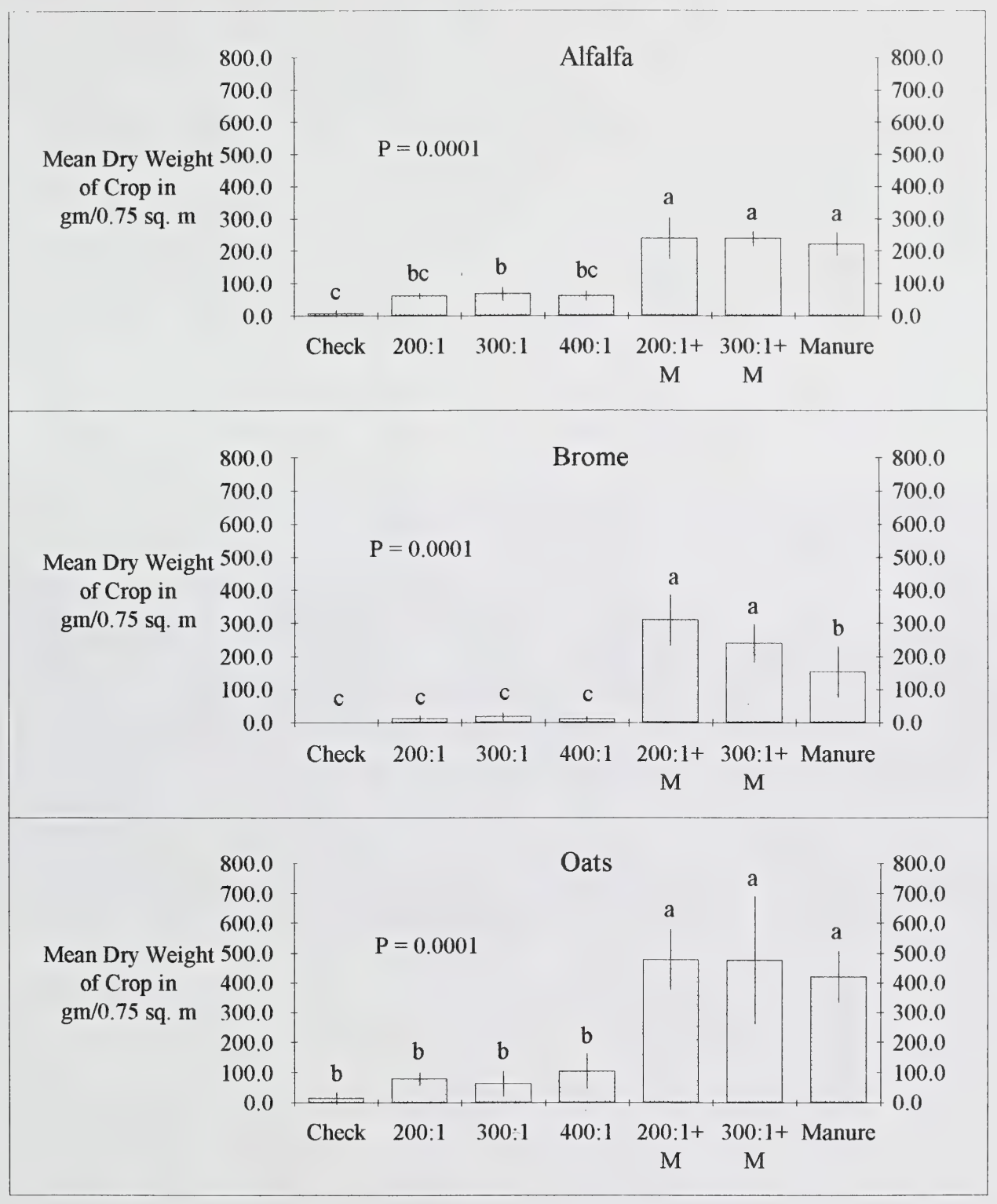

Figure 23. Treatment comparison on the Devon soil - fall 1991 harvest. Data shown as mean dry weight of alfalfa, brome and oats in gm/0.75 sq.m $(n=4)$. Bars with the same letter are not significantly different according to Duncan's Multiple Range Test. Lines represent standard deviation. 


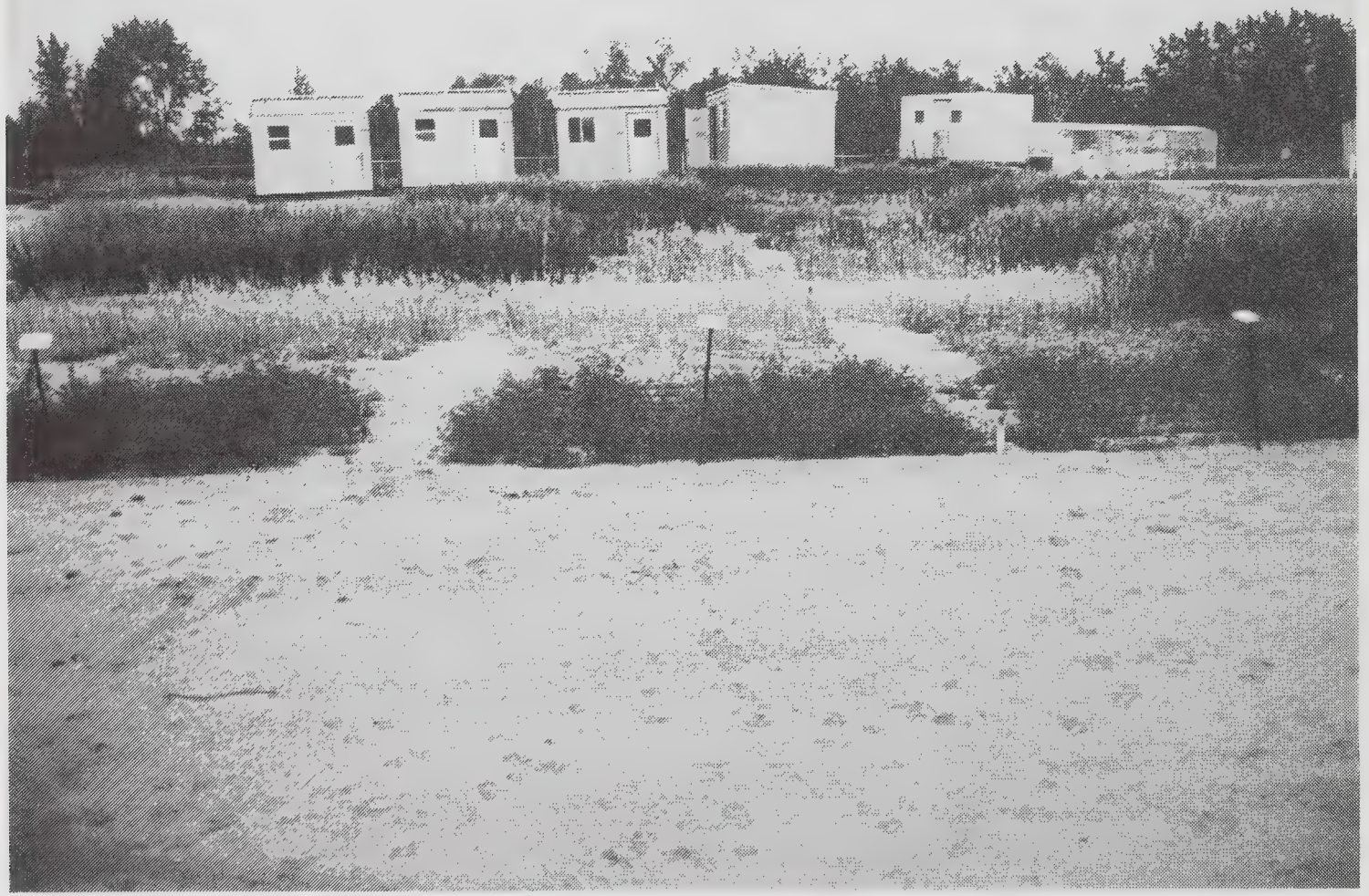

Figure 24. Photograph of alfalfa (foreground), bromegrass (middle), and oats (background) grown in loamy sand soil with atrazine residues at Devon. Activated charcoal was incorporated to a $30 \mathrm{~cm}$ depth (left to right) at a charcoal to herbicide active ingredient ratio of 200:1, 300:1, and 400:1. 


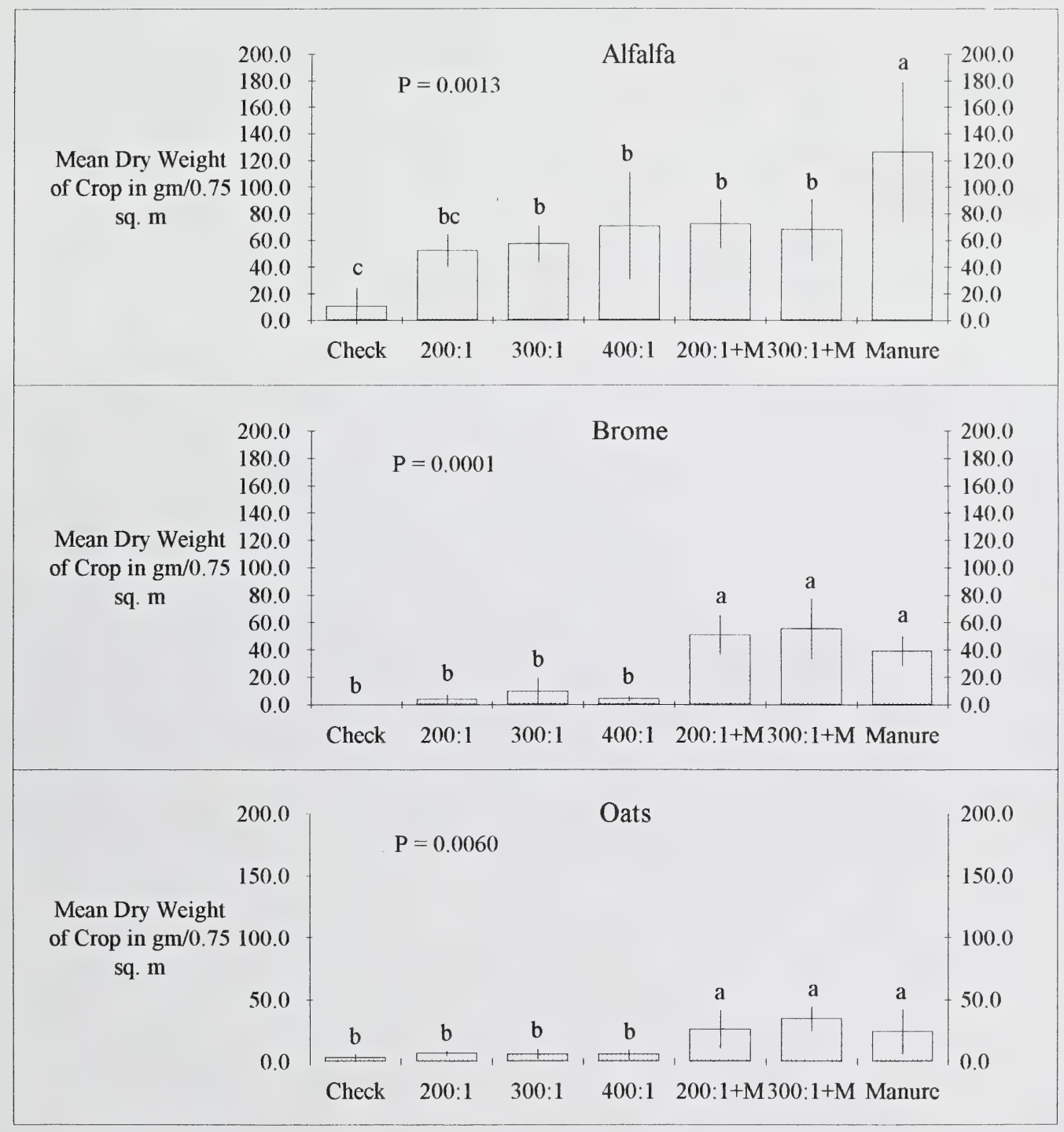

Figure 25. Treatment comparison on the Devon soil - fall 1992 harvest. Data shown as mean dry weight of alfalfa, brome, and oats in gm/ 0.75 sq.m. $(n=4)$. Bars with the same letter are not significantly different according to Duncan's Multiple Range Test. Lines represent standard deviation. 


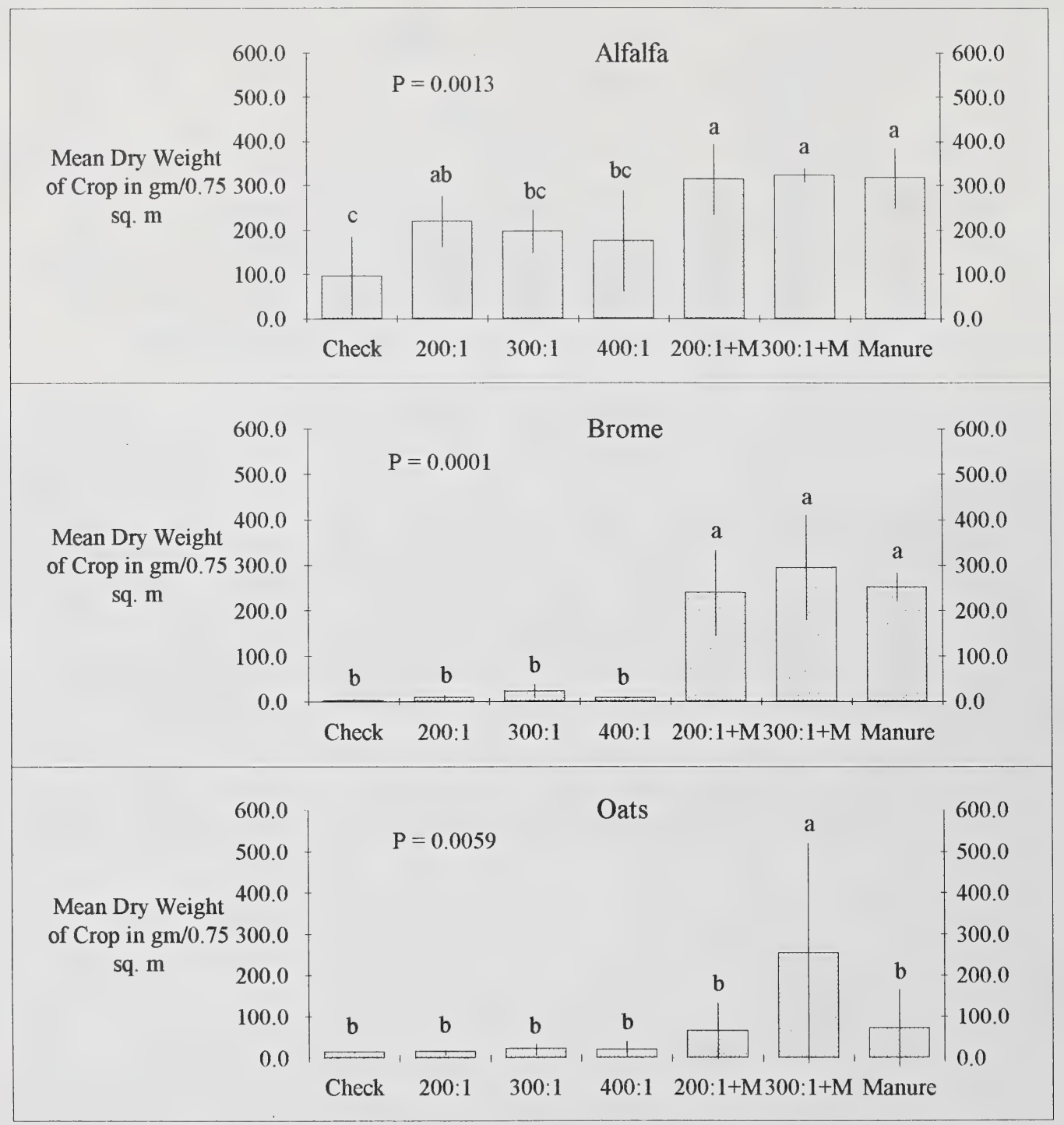

Figure 26. Treatment comparison on the Devon soil - fall 1993 harvest. Data shown as mean dry weight of alfalfa, brome, and oats in gm/0.75 sq.m. $(n=4)$. Bars with the same letter are not significantly different according to Duncan's Multiple Range Test. Lines represent standard deviation. 


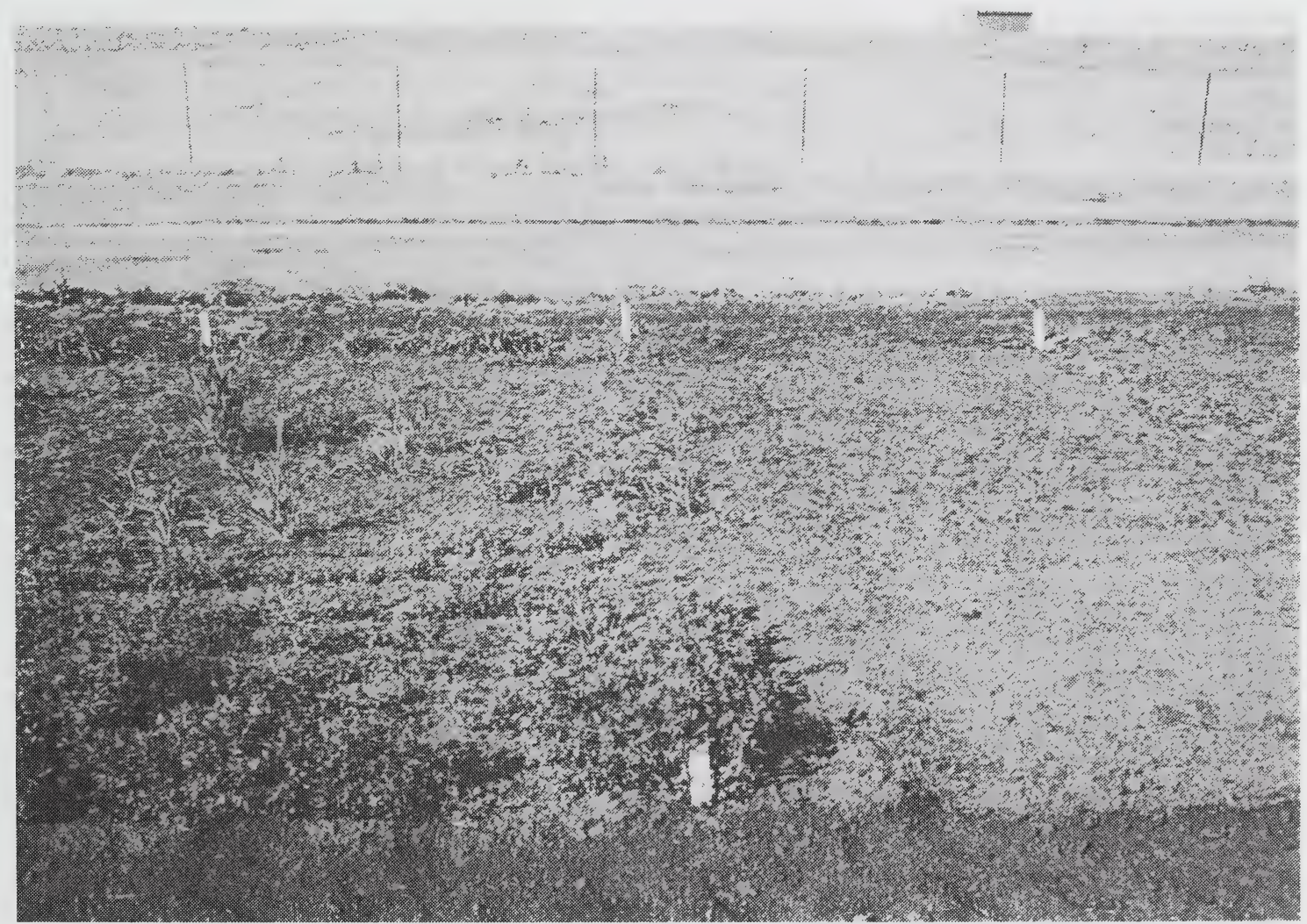

Figure 27. Photograph of alfalfa (foreground), oats (middle), and bromegrass (background) grown in soil treated with bromacil at $6 \mathrm{~kg} / \mathrm{ha}$ at Vegreville in the first growing season. Right plot: 1 to 2 year old manure alone ( $25 \mathrm{~cm}$ application depth); Left plot: activated charcoal at a ratio of $200: 1$ (1200 kg/ha of charcoal: $6 \mathrm{~kg} / \mathrm{ha}$ of bromacil) plus 1 to 2 year old manure $(25 \mathrm{~cm}$ application depth). 
with the low rate of herbicide, and no growth on the plots with the high rate of herbicide. The plants that established on the soil with the low levels of herbicide had herbicide injury.

In the third year (1993) the manure had decomposed further in the soil. Very small fibres of straw remained visible in the manure. The crop growth on all manure plus charcoal treatments remained excellent (Figure 21 and 22). Oats and brome grew in soil with the low bromacil rate and the manure alone treatment. No herbicide injury was evident. On the plots with the high bromacil rate and the manure alone no alfalfa grew, while oats and bromegrass grew poorly. All three crops grew well on the plots with the low rate of tebuthiuron and the manure alone treatment. At the high tebuthiuron rate and the manure alone treatment oats grew well, but brome and alfalfa grew poorly. The application rate of manure was slightly higher in the tebuthiuron plots than in the bromacil plots $(30 \mathrm{~cm}$ vs $20 \mathrm{~cm})$. This may have affected the response of the crops to the manure treatment, either by the higher manure rate providing more adsorptive surfaces and/or more microbial degradation. It may also have been a simple matter of the added volume of the manure diluting the herbicide concentration sufficiently to allow more growth.

\subsubsection{Devon}

At Devon in 1991, all treatments prevented herbicide injury. The treatments with manure produced plants with greater biomass than the treatments of charcoal alone (Figure 23 and 28). Slight herbicide injury (chlorosis) was noted at the end of the growing season in the manure alone treatments. During the 1992 growing season the manure treatments again produced plants with greater biomass than the treatments of charcoal alone (Figure 25). However, all of the treatments showed symptoms of herbicide injury (chlorosis) in the perennial crops. There was no injury to the oats. Upon excavation of the root systems it was noted that the roots of the perennial crops penetrated beyond the $30 \mathrm{~cm}$ treatment area, and were likely picking up herbicide from below $30 \mathrm{~cm}$. In preliminary soil sampling and bioassays, herbicide residues were found to a depth of $120 \mathrm{~cm}$. The $30 \mathrm{~cm}$ treatment area was chosen because it was thought to be the primary rooting area of oats and bromegrass, and financial limitations on the field work prohibited a deeper treatment area.

In the third growing season (1993) the manure treatments again produced plants with greater biomass than the treatments of charcoal alone (Figure 26), however there was no herbicide injury in the bromegrass, and only very slight injury (chlorosis) in a few of the replicates of alfalfa. The roots of the perennials were again excavated and the root length was much the same as it was in 1992. The oats grown on the control (no amendment) in the third year had no injury, and the growth was very similar to the plots with the treatments of charcoal alone 


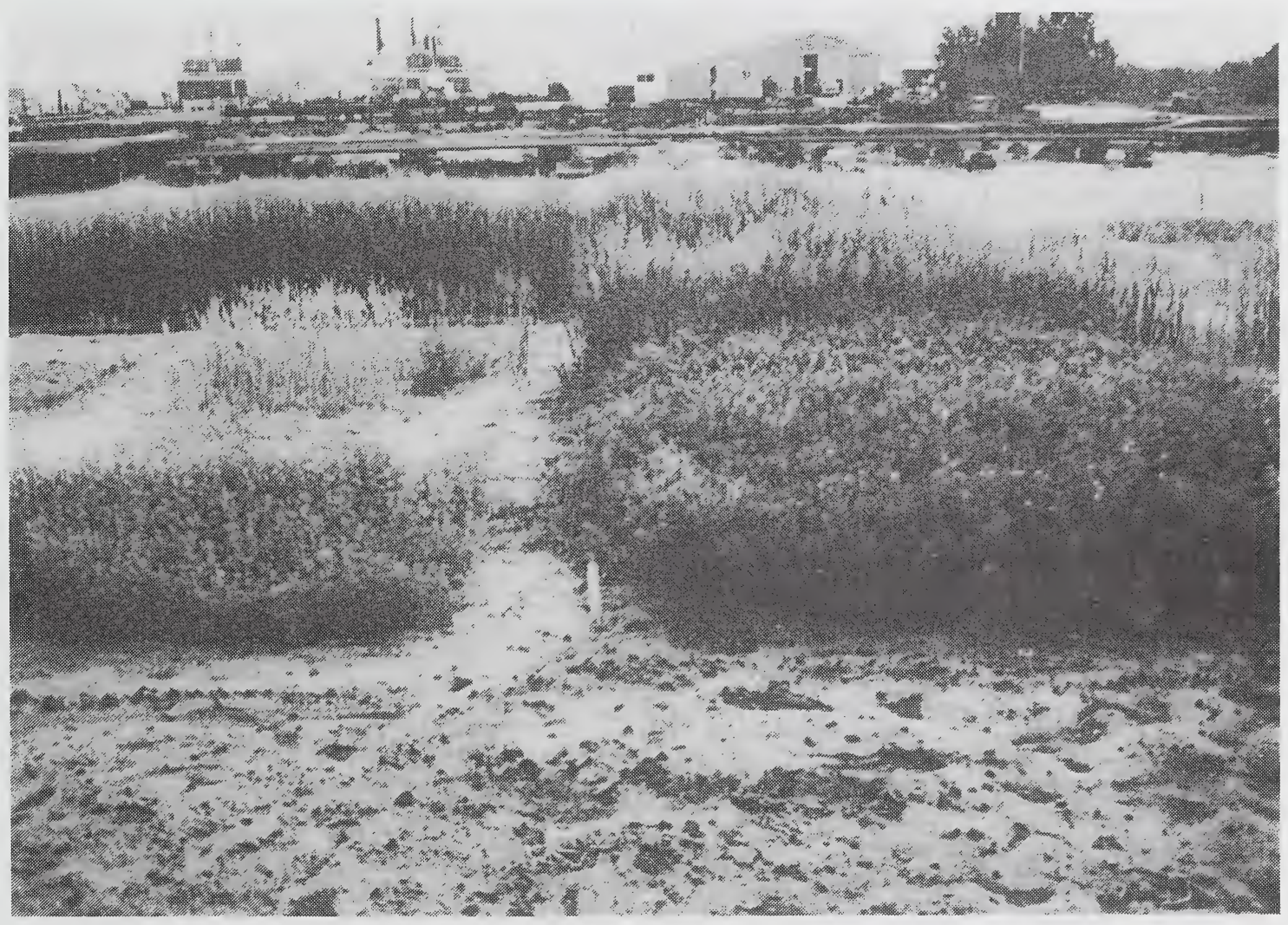

Figure 28. Photograph of alfalfa (foreground), bromegrass (middle), and oats (background) grown in loamy sand soil with atrazine residues at Devon in the first growing season. Left plot: ratio of activated charcoal:herbicide of 300:1; Right plot: ratio of activated charcoal:herbicide $300: 1$ plus 5 year old manure (12 cm application depth). 
suggesting that the herbicide residues in these plots were no longer phytotoxic. It was speculated that the herbicide had been degraded, or leached out of the root zone.

There was a difference in the effectiveness of the manure treatments between Vegreville and Devon. The success of the manure treatments at Devon was attributed to better manure quality (refer to Table 8 for manure composition), although it is acknowledged that other factors such as soil type, herbicide type, and herbicide rate could also play a role.

\section{3}

\section{WELL COMPOSTED MANURE VS PEAT PLUS FERTILIZER TREATMENTS}

This experiment was designed to determine what properties make manure an effective amendment, and to determine if a suitable alternative could be found for manure. The treatments were: (1) manure; (2) peat; (3) fertilizer; (4) combinations of charcoal and well composted manure; and (5) a combination of charcoal plus peat plus fertilizer. There are variations in manure quality and a combination of peat and fertilizer which would provide a more consistent amendment for reclamation. It would also provide an option in the event that a well composted manure was not available. This experiment was conducted at both Devon and Vegreville, to determine if well composted manure would be effective at Vegreville on the bromacil and tebuthiuron residues. Because of the textural difference between the two locations only fertilizer was used as a substitute (for manure) at Vegreville. Peat was used to replace the water holding capacity, and the adsorptive capacity of the manure, and to add to the soil structure by reducing the compaction on the industrial site. The fertilizer was used to replace the nutrients that were made available to the crops by the manure. It was evident that both of these properties of manure might be beneficial at Devon on the Loamy Sand soil, where the organic matter was less than one percent. However, on the Silt Loam soil at Vegreville where the organic matter was ten percent it was initially thought that the fertilizer alone would be a suitable substitute.

In the 1992 growing season the crops grown in the low herbicide levels at Vegreville were adequately protected by well composted manure alone, charcoal alone, and the combination of charcoal plus manure as reflected in the biomass measurements reported in Figure 29. No herbicide injury was present in these treatments. The charcoal plus fertilizer, and fertilizer alone produced crops with similar biomass however, herbicide injury was present on all of the crops. A local source of well composted cattle manure approximately 10 years old was used (refer to manure composition Table 8). The manure was applied in a 10 to $15 \mathrm{~cm}$ layer. The fertilizer applied was equivalent to the available $\mathrm{N}, \mathrm{P}$, and $\mathrm{K}$ in the local manure (refer to rates of application Table 9). On the high herbicide residue levels no significant difference was found between any of the treatments at $\mathbf{P}=0.05$ (refer to Figure 30). 


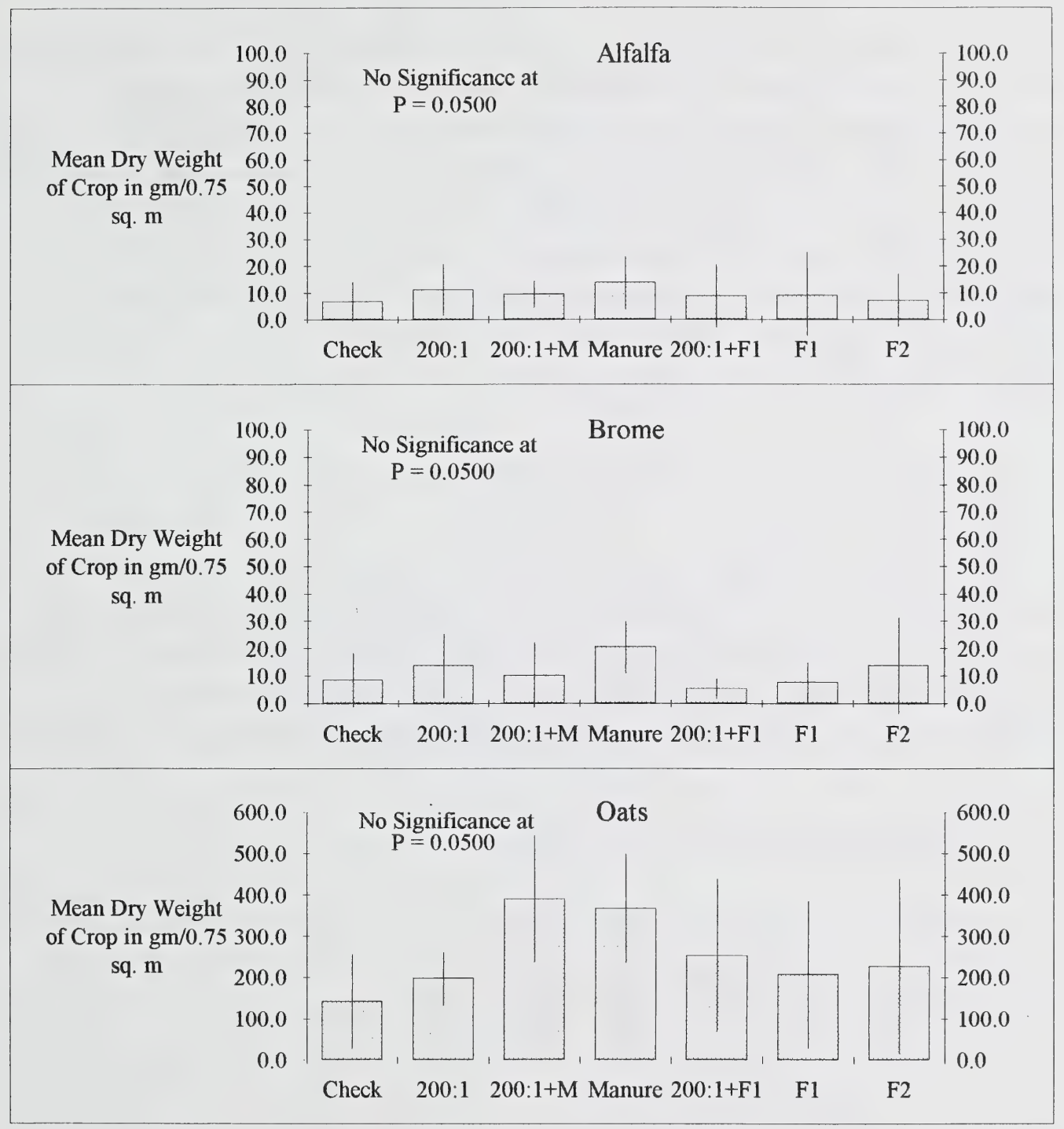

Figure 29. Treatment comparison on the Vegreville soil with initial bromacil residues of $2 \mathrm{~kg} / \mathrm{ha}$ fall 1992 harvest. Data shown as mean dry weight of alfalfa, brome and oats in gm/0.75 sq.m. $(n=4)$. Bars with the same letter are not significantly different according to Duncan's Multiple Range Test. Lines represent standard deviation 
In the 1993 growing season crop growth on the control plots (herbicide - no amendment) indicated bromacil and tebuthiuron residues were no longer phytotoxic (data not shown).

At Devon in the 1992 growing season the charcoal plus manure, and charcoal plus fertilizer plus peat treatments provided effective protection against herbicide injury and demonstrated the best crop growth as reflected in the biomass measurements in Figure 31. Manure alone, and peat plus fertilizer treatments also provided effective protection against herbicide injury and demonstrated adequate crop growth. Similar trends were evident for the 1993 harvest (Figure 32), except that charcoal alone and peat alone did very poorly. Charcoal alone, fertilizer alone, peat alone, and charcoal plus fertilizer provided some protection against herbicide injury but showed poorer crop growth. The same cattle manure source was used in this experiment as was used in the last Devon experiment, only the manure was an additional year older.

In a another attempt to explain the effect of manure quality, an experiment conducted at Devon compared 10 year old cattle manure to five year old manure (refer to Table 8 for manure composition). Both manures adequately protected all three crops from herbicide injury, however the 10 year old manure produced the plants with the greater biomass (as reflected in Figures 33 and 34). The results were similar in both the 1992 and the 1993 growing season.

Efficiency of activated charcoal incorporation is very important, and it will affect the outcome of the reclamation process (Ogg 1982). It was speculated that the more thorough the incorporation the more likely the success. An experiment investigating the efficiency of treatment incorporations with a rotovator was initiated at Devon. One, two and four incorporations were compared in a single season to optimize the number of incorporations, and to see which would be best. Two ratios of charcoal to herbicide $(100: 1,200: 1)$ were examined to see if lower ratio with more incorporations would be as effective as the higher ratio and fewer incorporations. The result of this experiment showed no significant differences $(P=0.05)$ between the number of incorporations or the ratio of charcoal to herbicide (data not shown). However, it is speculated that if a less efficient machine or tool were used to incorporate, such as a disking unit or a hoe (as have been used in much of the literature), that the number of incorporations may be a factor. 


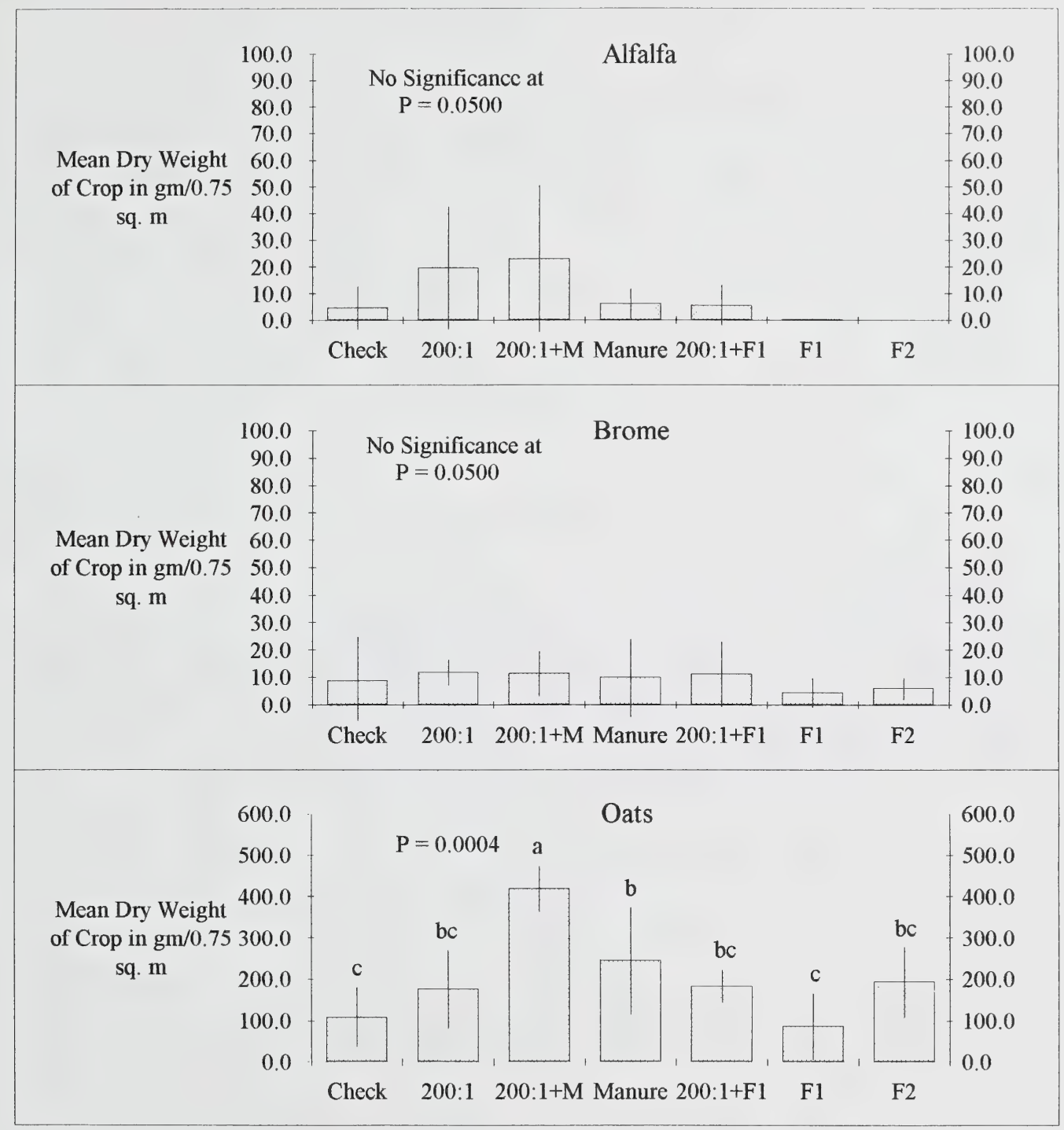

Figure 30. Treatment comparison on the Vegreville soil with initial bromacil residues of $6 \mathrm{~kg} / \mathrm{ha}$ fall 1992 harvest. Data shown as mean dry weight of alfalfa, brome and oats in gm/0.75 sq.m. (n =4). No significant difference was found between any of the treatments according to Duncan's Multiple Range Test. Lines represent standard deviation. 


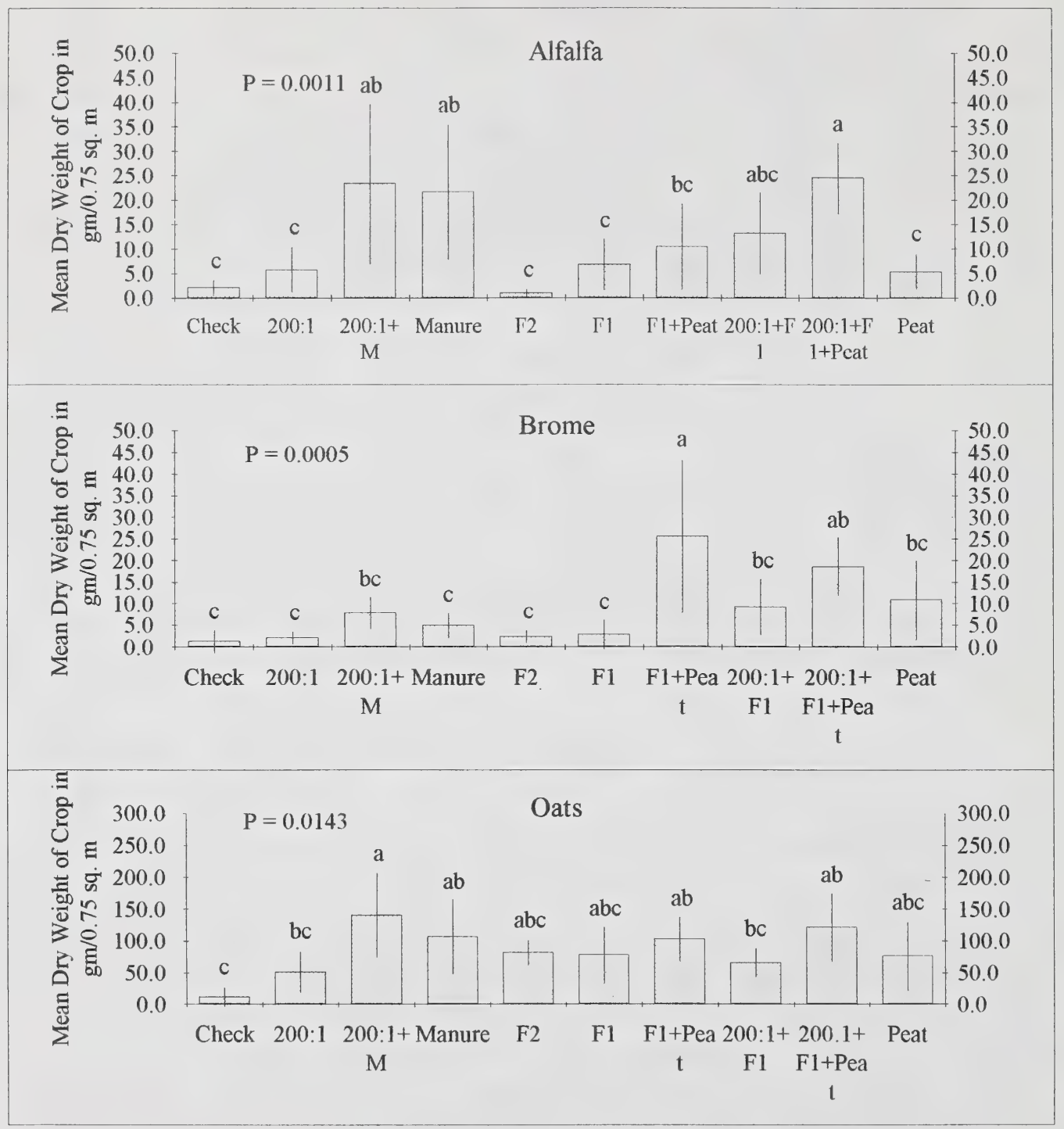

Figure 31. Devon 1992 field experiment - fall 1992 harvest. Data shown as mean dry weight of alfalfa, brome and oats in gm/0.75 sq.m. $(n=4)$. Bars with the same letter are not significantly different according to Duncan's Multiple Range Test. Lines represent standard deviation. 


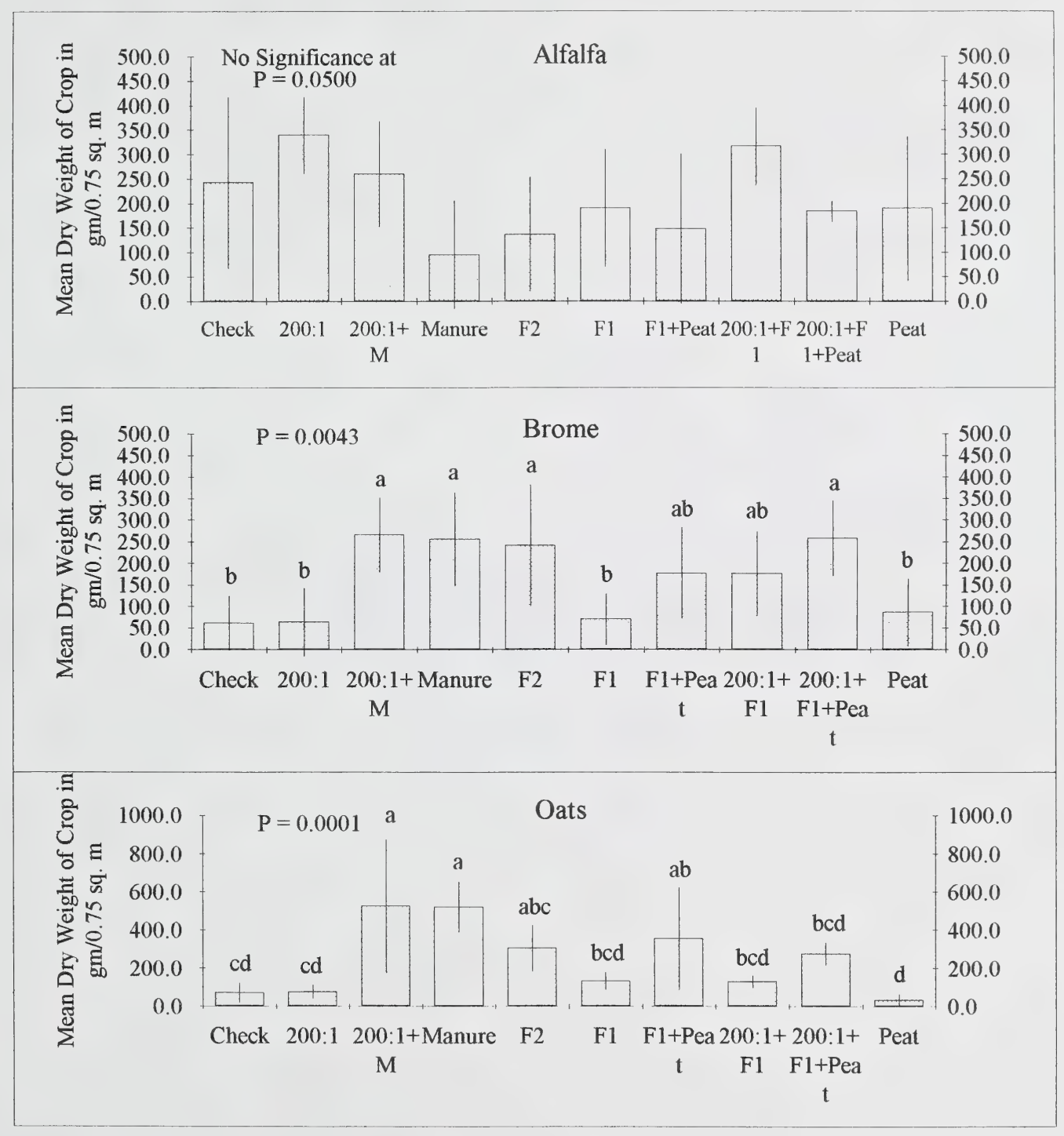

Figure 32. Devon 1992 field experiment - fall 1993 harvest. Data shown as mean dry weight of alfalfa, brome and oats in gm/0.75 sq.m. $(\mathrm{n}=4)$. Bars with the same letter are not significantly different according to Duncan's Multiple Range Test. Lines represent standard deviation. 


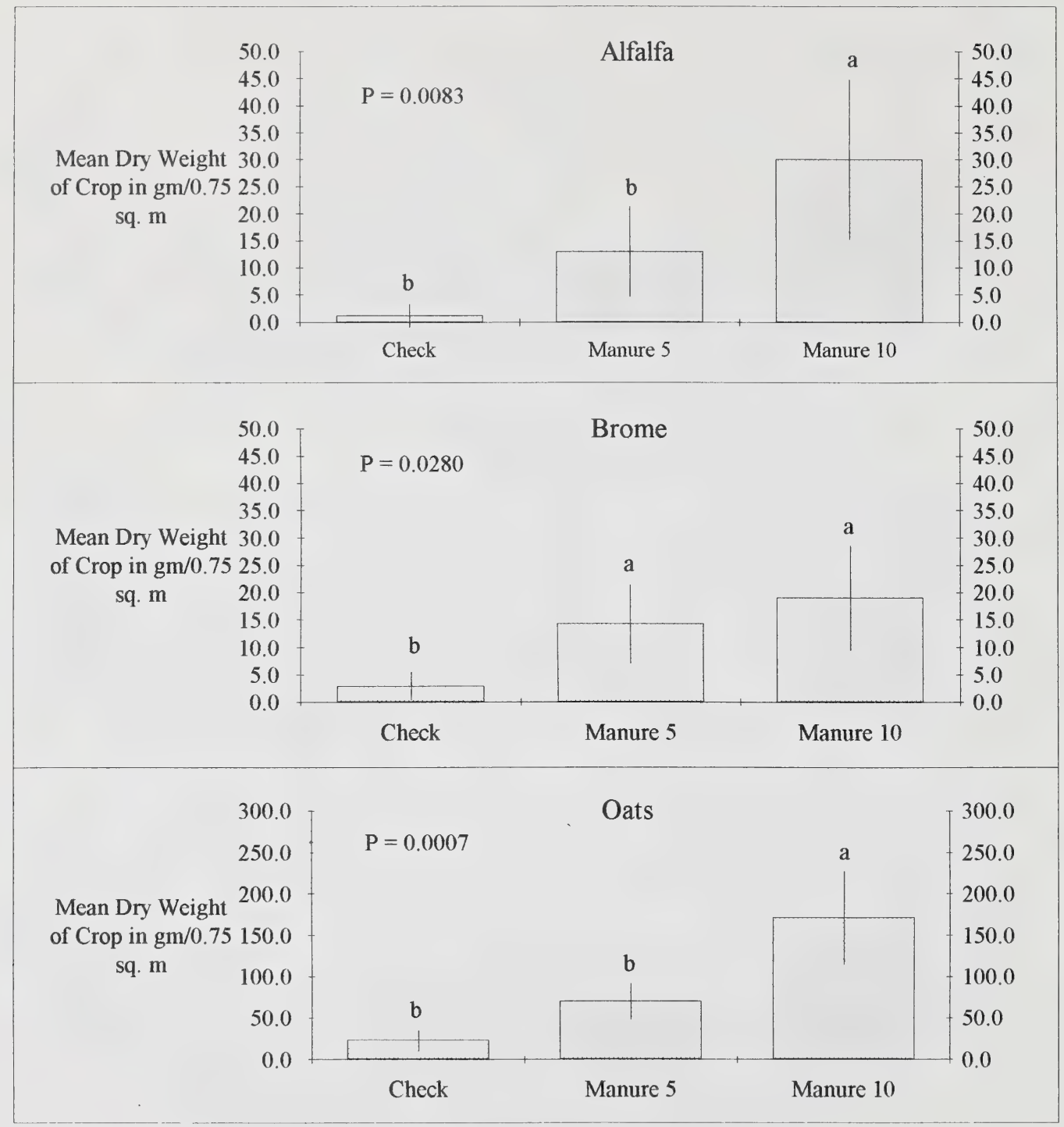

Figure 33. Manure comparison experiment at Devon - fall 1992 harvest. Data shown as mean dry weight of alfalfa, brome and oats in gm/ 0.75 sq.m. $(n=4)$. Bars with the same letter are not significantly different according to Duncan's Multiple Range Test. Lines represent standard deviation. 


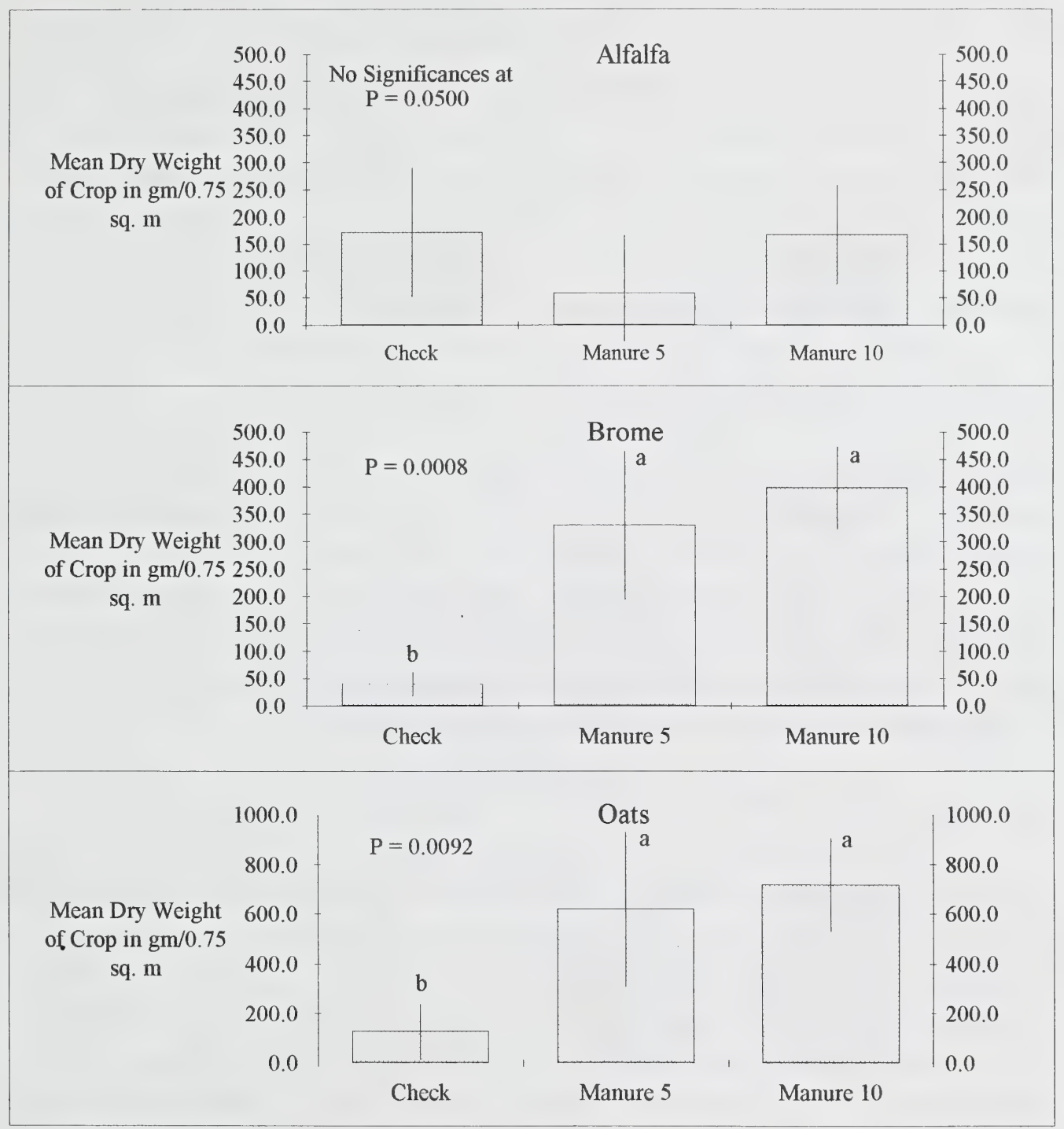

Figure 34. Manure comparison experiment at Devon - fall 1993 harvest. Data shown as mean dry weight of alfalfa, brome and oats in $\mathrm{gm} / 0.75$ sq.m. $(\mathrm{n}=4)$. Bars with the same letter are not significantly different according to Duncan's Multiple Range Test. Lines represent standard deviation. 
At Devon on the loamy sand soil, the soil moistures on the plots without organic amendments ranged from $10 \%$ to $12 \%$. Peat increased the soil moisture to $12 \%$ to $22 \%$. Manure increased the soil moisture to $20 \%$ to $28 \%$. At Vegreville (silt loam soil) the soil moistures on the plots without organic amendments ranged from $12.5 \%$ to $15.5 \%$. Manure increased the soil moisture to $18 \%$ to $22 \%$.

At Vegreville the $\mathrm{pH}$ of the soil was unaffected by the addition of any of the amendments. The manure treatments caused a slight increase in the $\mathrm{pH}$ at the Devon site.

Manure increased the electrical conductivity (EC) of the soil at Devon by 0.4 to $0.7 \mathrm{dS} / \mathrm{cm}$, however the soil was still in an acceptable range (below $1 \mathrm{dS} / \mathrm{cm}$ ). The fertilizer did not affect the EC at Devon. The EC of the soil increased at Vegreville with the addition of both manure and fertilizer as much as $1.5 \mathrm{dS} / \mathrm{cm}$ in the bromacil plots. The measurements were complicated in the tebuthiuron plots by naturally high EC levels, although in general it appeared that the manure and fertilizer treatments caused a significant increase in EC.

The increased soil moisture content caused by the addition of the peat and manure helps explain the increased biomass found on the plots with these treatments. This is especially true at the Devon site which had low moisture holding capacity. The changes in $\mathrm{pH}$ and $\mathrm{EC}$ were not large enough to have caused any negative treatments effects.

\subsection{TREATMENT COST COMPARISON}

The costs of the materials used in the field study were as follows: (1) Well composted manure $\sim \$ 16 \mathrm{~m}^{3}$ (included hauling); (2) Sphagnum peat $\sim \$ 20 \mathrm{~m}^{3}$ (included hauling); (3) Fertilizer $\sim \$ 15$ per $25 \mathrm{~kg}$; (4) Activated charcoal $\sim \$ 1.70 / \mathrm{kg}$. The manpower to apply and incorporate each of these amendments would be more or less equivalent for each treatment.

As an example, if cost estimates from our study are used, materials (only) for a treatment area of $1000 \mathrm{~m}^{2}$ with residue levels of $0.8 \mathrm{ppm}(2 \mathrm{~kg} / \mathrm{ha})$ to a depth of $15 \mathrm{~cm}$ would be as follows:

1. Activated charcoal.

Cost: $\sim \$ 1.70 / \mathrm{kg}$

Required volume (assume ratio of charcoal to herbicide 200:1): $400 \mathrm{~kg}$

Cost for $1000 \mathrm{~m}^{2}: \sim \$ 680$

2. Well composted manure.

Cost: $\sim \$ 16 \mathrm{~m}^{3}$

Required volume: $150 \mathrm{~m}^{3}$ 
Cost for $1000 \mathrm{~m}^{2}: \sim \$ 2250$

3. Activated charcoal plus well composted manure.

Cost for $1000 \mathrm{~m}^{2}: \sim \$ 2930$

4. Sphagnum peat plus fertilizer.

Cost of peat: $\sim \$ 20 \mathrm{~m}^{3}$

Cost of fertilizer: $\sim \$ 15$ per $25 \mathrm{~kg}$

Required materials: $150 \mathrm{~m}^{3}$ of peat $+\sim 250 \mathrm{~kg}$ of fertilizer

Cost for $1000 \mathrm{~m}^{2}: \sim \$ 3150$

5. Activated charcoal plus peat plus fertilizer.

Cost for $1000 \mathrm{~m}^{2}: \sim \$ 3830$

The best crop biomass would most likely come from the activated charcoal plus well composted manure treatment at a cost of $\sim \$ 2930$.

Using this same example, if herbicide residue levels were $2.4 \mathrm{ppm}(6 \mathrm{~kg} / \mathrm{ha})$, the cost of materials (only) would be as follows:

1. Activated charcoal.

Required volume (assume ratio of charcoal to herbicide 200:1): $1200 \mathrm{~kg}$

Cost for $1000 \mathrm{~m}^{2}: \sim \$ 2040$

2. Activated charcoal plus well composted manure.

Cost for $1000 \mathrm{~m}^{2}: \sim \$ 4290$

3. Activated charcoal plus peat plus fertilizer.

Cost for $1000 \mathrm{~m}^{2}: \sim \$ 5190$

Again, the best crop biomass would most likely come from the activated charcoal plus well composted manure treatment. The cost would be about $\sim \$ 4290$. Manure (alone), and peat plus fertilizer (alone) would probably be ineffective at preventing herbicide injury at this residue level.

If the depth of herbicide residues was $30 \mathrm{~cm}$, the cost of materials would double for the $1000 \mathrm{~m}^{2}$. The cost of the amendment application would also increase because the upper soil layer would have to be stripped to apply the amendments to the sub-surface, and the upper layer would have to be replaced. 
6. CONCLUSIONS

1. At the low bromacil or tebuthiuron levels treatments of manure alone, and manure plus activated charcoal $(200: 1)$ provided the best protection from herbicide injury on the silt loam Chernozem top soil. They were not effective at the high herbicide rates.

2. The commercial humates, sawdust, commercial microbes, fly ash, and waste activated charcoal were not effective at either bromacil or tebuthiuron rate on the silt loam top soil.

3. Activated charcoal alone and activated charcoal plus manure at a charcoal to herbicide (a.i.) ratio of 200:1 provided satisfactory plant protection at herbicide concentrations ranging from 0.71 to $1.2 \mathrm{ppm}$ a.i. on the loamy sand sub-soil. The commercial microbes were not tested on this soil due to insufficient soil quantities.

4. Activated charcoal at a charcoal to herbicide ratio of $100: 1$, manure alone, commercial humates, sawdust, fly ash, and waste activated charcoal were not effective at atrazine levels of $2.2 \mathrm{ppm}$ a.i. on the loamy sand sub-soil.

5. A visual assessment of herbicide injury using plant bioassays (oats and alfalfa) indicated phytotoxic levels of herbicides on the Devon site to a depth of at least $1.2 \mathrm{~m}$ in the loamy sand sub-soil.

\subsection{FIELD STUDIES}

1. Activated charcoal prevents herbicide injury on herbicide treated silt loam and loamy sand Chernozems under Alberta field conditions. It can be effective under field conditions at a ratio of activated charcoal to herbicide active ingredient of 200:1.

2. Under field conditions there was no significant difference between activated charcoal to herbicide active ingredient ratios of $200: 1,300: 1$, and 400:1 on a silt loam or a loamy sand soil.

3. Activated charcoal in combination with a well composted manure is an effective soil amendment on herbicide treated silt loam or loamy sand soil. 
4. Crops grown on soil amended with charcoal plus manure produced more biomass than plants grown on soil amended with either manure or charcoal alone.

5. The quality (age) of manure affects its ability to prevent herbicide injury. Well composted manure ( $>5$ years old) is an effective soil amendment for low levels $(2 \mathrm{~kg} / \mathrm{ha})$ of bromacil and tebuthiuron on a silt loam soil, and for levels of atrazine as high as $5 \mathrm{~kg} / \mathrm{ha}$ on a loamy sand soil. It is not an effective amendment at $6 \mathrm{~kg} / \mathrm{ha}$ of either bromacil or tebuthiuron on a silt loam soil. Manure less than 2 years old does not protect plants from herbicide injury even at herbicide concentrations $<2 \mathrm{~kg} / \mathrm{ha}$.

6. Peat plus fertilizer as an amendment provided equivalent protection from herbicide injury, and similar plant growth as manure alone in a loamy sand soil.

7. Activated charcoal plus peat plus fertilizer provided equivalent protection from herbicide injury, and similar plant growth as activated charcoal plus well composted manure in a loamy sand soil.

8. Plants grown in soil with treatments of activated charcoal plus well composted manure or activated charcoal plus peat plus fertilizer have greater biomass than plants grown on soil amended with either well composted manure or peat plus fertilizer alone.

9. The roots of the perennial crops were found to be below the $30 \mathrm{~cm}$ treatment area on the loamy sand sub-soil at Devon, and consequently showed herbicide injury symptoms. When phytotoxic levels of herbicides exceed 30 $\mathrm{cm}$ it is necessary to reclaim beyond $30 \mathrm{~cm}$ if a perennial crop is to become established. 
Although this project was one of applied research, one of the biggest questions relating to herbicide sorption is the fate of the bound residue. It is not known if the sorbed herbicides can become desorbed and become active in the soil again, and it is not known if the herbicide becomes protected from chemical and microbial degradation or is more susceptible. Bollang (1991) reported that pesticides bound to humus usually do not present a hazard to the environment, because the chemical linkages formed are quite stable and resistant, however the potential for release is still present. Coffey and Warren (1969) investigated desorption, found that of seven herbicides $(2,4-\mathrm{D}$, diphenamid, chlorthal-dimethyl, trifluralin, amiben, chlorpropham, dinoseb) there was evidence of desorption of the herbicide from the activated charcoal in all but dinoseb. The lack of dinoseb desorption may have been more of a function of the bioassay which may not have been sensitive enough to detect the small amount of herbicide desorbed.

Several authors (Harvey 1973; Hoagland 1989; Shea 1985) have reported that the use of activated charcoal as a soil amendment reduces the degradation of herbicides. Interactions of pesticides with humic substances have been reported by Saint-Fort and Visser (1988) to decrease the biological availability of the pesticide, and decrease the potential of environmental pollution. Klowkowski and Fuhr (1987) concluded that the incorporation of aged herbicide residues in the high molecular weight and stable humin fraction leads to a reduction of their bioavailability in comparison to treatments containing freshly applied substance. Khan (1991) stated that bound residues may become available for uptake by plants, however he reported that availability of residues to plants is considerably lower from bound residues than from freshly pesticide-treated soil.

A steady increase in the non-extractable fraction of herbicide residues remaining in the soil over time was found by Dao and co-workers (1979). It is therefore possible that the relationship between bioavailability of the bound residues and non-availability is related to the time following the herbicide application. Freshly bound residues appear more available, than "aged" residues.

In all, not much information is currently available pertaining to the nature and potential biological activity of the chemicals that are bound in soil, and more research, both fundamental and applied in this field is desirable.

When sterilant residues exceed $30 \mathrm{~cm}$ in depth, incorporation of amendments beyond $30 \mathrm{~cm}$ may be required. In order to determine the required depth of reclamation, longer term studies of crop growth should be considered. 
8.1.

\section{PVC SAMPLING METHOD}

The soil sampling and testing method was developed by the Alberta Environmental Centre Weed Science Section for detecting herbicide carryover on crop land. The samples were taken by hand using a mallet with a hollow $5 \times 40 \mathrm{~cm}$ long PVC tube (Figure 35). Holes were pre-drilled through the side of the tube at $8 \mathrm{~cm}$ intervals. Oats (Avena sativa L.) were seeded as an indicator species with three seeds in each hole. The hole spacing is important to prevent the roots of the seedlings from growing into each other. The soil sterilants cause death or injury of the plants which are quite distinct from the symptoms of poor growth that may occur due to the nature of the subsoil material. The tubes are then set into a growth chamber for $16 \mathrm{~h}$ at $22^{\circ} \mathrm{C}$ and for $8 \mathrm{~h}$ at $16^{\circ} \mathrm{C}$. The tubes are kept moist by watering twice daily. In the 2 nd and 4 th week the plants were scored for herbicide injury on a 0 to 9 scale where $0=$ complete kill, and $9=$ no visible effect.

8.2. SCALE FOR CROP TOLERANCE

9 Complete tolerance - no effect of the herbicide

8 Possible effect

$7 \quad$ Slight effect - Initial injury slight; good recovery

6 Definite effect - Initial injury moderate; fair recovery

4-5 Severe effect - Initial injury severe; poor recovery

1-3 Severe effect - Initial injury severe; only slight recovery

$0 \quad$ Complete kill 


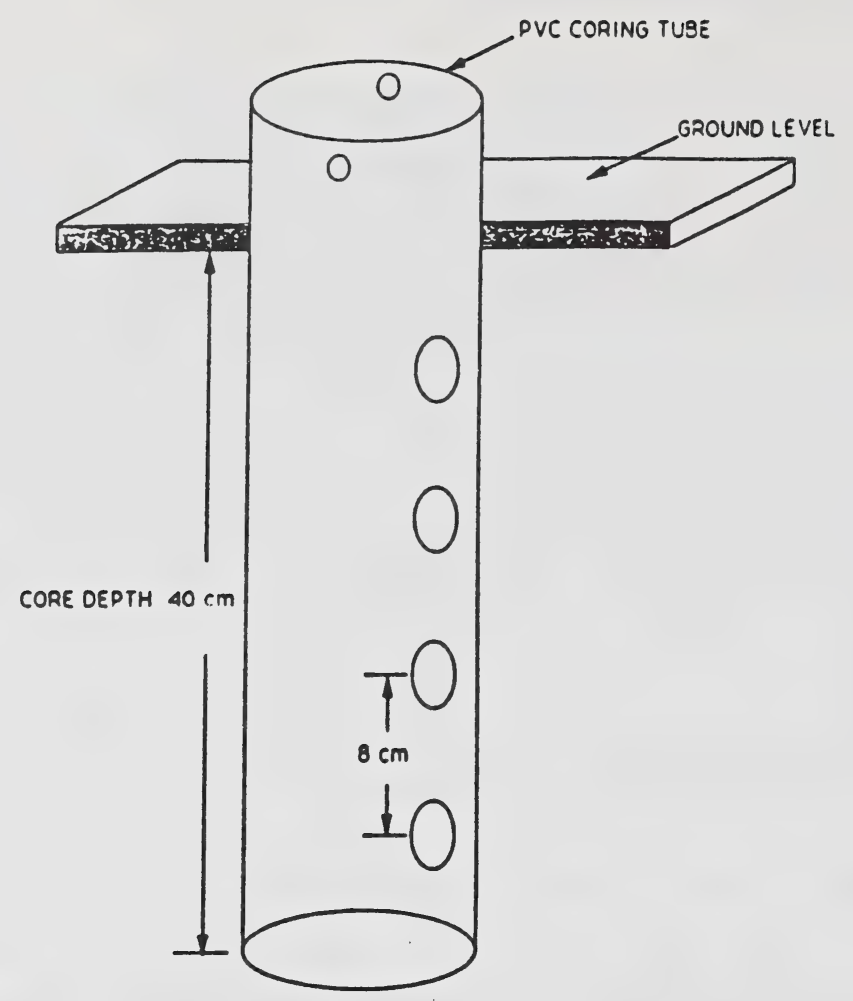

1. How Soll Sample is Taken With PVC Tube

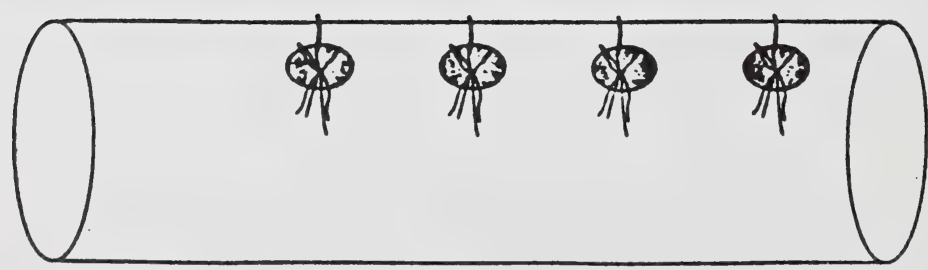

2. Illustration of PVC - Bioassay

Figure 35. Illustration of a PVC tube for bioassays. 
Aitken, R.L. and L.C. Bell, 1985. Plant uptake and phytotoxicity of boron in Australian fly ashes. Plant and Soil 84: 245-257.

Ahrens, J.F., 1965. Detoxification of simazine and atrazine treated soil with activated carbon. IN: Proceedings of the Northeast Weed Control Conference 19: 364.

Albainis, T.A., Pomonis, P.J. and A.T. Sdoukos, 1988. The influence of fly ash on hydrolysis, degradation and adsorption of methyl parathion in aqueous soil suspensions. Toxicological and Environmental Chemistry 17: 351-362.

Albainis, T.A., Pomonis, P.J. and A.T. Sdoukos, 1989a. The influence of fly ash on Pesticide fate in the environment. 1. hydrolysis, degradation and adsorption of lindane in aqueous mixtures of soil with fly ash. Toxicological and Environmental Chemistry 19: 161169.

Albainis, T.A., Pomonis, P.J. and A.T. Sdoukos, 1989. The influence of fly ash on Pesticide fate in the environment. II. hydrolysis, degradation and adsorption of atrazine in aqueous mixtures of soil with fly ash. Toxicological and Environmental Chemistry 19: 171178 .

Andersen, A.H., 1968. The inactivation of simazine and linuron in soil by charcoal. Weed Research 8: 58-60.

Ashley, R.A., and E.M. Rahn, 1967. Persistence of atrazine at two locations as affected by soil incorporation and certain additives. IN: Proceedings of the Northeast Weed Control Conference 21: 557.

Bollag, J., 1991. Enzymatic binding of pesticide degradation products to soil organic matter and their possible release. Chapter 9. American Chemical Society p. 122-132.

Bovey, R.W., and F.R. Miller, 1969. Effect of activated carbon on the phytotoxicity of herbicides in a tropical soil. Weed Science 17: 189-191.

Campbell, D.J., Fox, W.E., Aitken, R.L., and L.C. Bell, 1983. Physical characteristics of sands amended with fly ash. Australian Journal of Soil Research 21: 147-154.

Campbell, C.A., Schnitzer, M., Stewar, J.W.B, Biederbeck, V.O. and F. Selles, 1986. Effect of manure and $\mathrm{P}$ fertilizer on properties of a black chernozem in southern Saskatchewan. Canadian Journal of Soil Science 66: 601-613.

Cheng. H.H. and R.G. Lehmann, 1985. Characterization of herbicide degradation under field conditions. Weed Science 33: 7-10. 
Coffey, D.L. and G.F. Warren, 1969. Inactivation of herbicides by activated carbon and other absorbents. Weed Science 17: 16-19.

Cotton, M.M. and M.P. Sharma, 1992. Reclamation of sterilant affected sites: A review of the issue in Alberta. Alberta Land Conservation and Reclamation Council Report No. RRTAC 92-1. ISBN 0-7732-0884-4. 64 pp.

Dao, T.H., Lavy, T.L., and R.C. Sorensen, 1979. Atrazine degradation and residue distribution in soil. Soil Science Society of America Journal 43: 1129-1134.

Duah-Yentumi, S. and S. Kuwatsuka, 1981. Effect of organic matter and chemical fertilizers on the degradation of benthiocarb and MCPA herbicides in the soil, Soil Science Plant Nutr. 26: 551-559.

Duah-Yentumi, S. and S. Kuwatsuka, 1982. Microbial degradation of benthiocarb, MCPA and 2,4-D herbicides in perfused soils amended with organic matter and chemical fertilizers. Soil Science Plant Nutr. 28: 19-26.

Eagle, D.J., 1988. Fate of applied pesticides in the environment. Aspects of Applied Biology (17) Environmental aspects of applied biology p. 209-212.

El-Mogai, D., Lisk, D.J., and L.H. Weinstein, 1988. A review of physical, chemical, and biological properties of fly ash and effects on agricultural ecosystems. The Science of the Total Environment 74: 1-37.

Feustel, I.C. and H.G. Byers, 1936. The comparative moisture-absorbing and moisture-retaining capacities of peat and soil mixtures. Technical bulletin, United States Department of Agriculture, Washington, No. 532: 26.

Foth, H.D., and B.G. Ellis, 1988. Soil Fertility. John Wiley \& Sons, Toronto. p. 14.

Grover, R., 1966. Influence of organic matter, texture, and available water on the toxicity of simazine in soil. Weeds 14: 148.

Grandmaison, J., 1982. The horticultural potential of sphagnum peats, as related to their physical and physio-chemical properties. Symposium on peat and peatlands: proceedings Sept. 12-15, 1982. Shippagan, NB, p. 324-366.

Guo, L., Bicki, T.J., Felsot, A.S., and T.D. Hinesly, 1991a. Phytotoxicity of atrazine and alachlor in soil amended with sludge, manure and activated carbon. Journal of Environmental Science and Health, B26: 513-527. 
Guo, L., Bicki, T.J., Hinesly, T.D., and A.S. Felsot, 1991b. Effect of carbon-rich waste materials on movement and sorption of atrazine in a sandy, coarse-textured soil. Environmental Toxicology and Chemistry 10: 1273-1282.

Gwyer, B.D., and J.A. Robertson, 1979. Fly ash as a soil amendment, Phase 1 - characterization. Department of Soil Science, The University of Alberta. May, 1979. pp.36.

Harvey, R.G., 1973. Influence of cropping and activated carbon on persistence of atrazine in sand. Weed Science 21: 204-206.

Hoagland, R.E., 1989. The use of activated carbon and other adsorbents as herbicide safeners. In: Crop safeners for herbicides: development, uses, and mechanisms of action. SanDiego, California, USA. p. 243-281.

Hoffman, G.L., Nikols, D.J., Stuhec, S., and R.A. Wilson, 1993. Evaluation of lenardite and humalite resources of Alberta. Energy, Mines and Resources C

Jordan, P.D., and L.W. Smith, 1971. Adsorption and deactivation of atrazine and diuron by charcoals. Weed Science. 19: 541-544.

Kenaga, E.U., 1980. Predicted bioconcentration factors and soil sorption coefficients of pesticides and other chemicals. Ecotoxicology and Environmental Safety. 4: 26-38.

Khan, S.U., 1991. Bound (non extractable) pesticide degradation products in soils: Bioavailability to plants. In: Pesticide Transformation Products: Fate and Significance in the Environment. Editors: Somasundaram, L. and J.R. Coats. ACS Symposium Series. Washington. p.108-121.

Kloskowsk, R., and F. Fuhr, 1987. Aged and bound herbicide residues in soil and their bioavailability. Journal of Environmental Science and Health B22: 623-642.

Koskinen, W.C., and S.S. Harper, 1990. The retention process: mechanisms. In: Pesticides in the soil environment: processes, impacts and modeling. Soil Science of America (SSSA Book Series, no.2), Madison, USA. p. 51-78.

Lamoreaux, R.J., Corbin, V.L., and B.S. Johl, 1989. Adsorption and deactivation of norflurazon by activated charcoal. Weed Technology 3: 297-302

LeBaron, H.M., 1970. Ways and means to influence the activity and the persistence of triazine herbicides in soils. Residue Review 32: 311-353

Logan, R.J., 1978. Peat as a soil amendment for tailing sand reclamation. University of Alberta, Edmonton, Alberta. M.Sc. Thesis. 197 pp. 
Macyk, T.M., 1983. Revegetation studies on fly ash and overburden at Grande Cache. Alberta Land Conservation and Reclamation Council Final Report No.79-1-MAC. pp.68.

Moyer, J.R., Hance, R.J., and C.E. McKone, 1972. The effect of adsorbents on the rate of degradation of herbicides incubated with soil. Soil Biol. Biochem. 4: 307-311.

Ndayegamiye, A., and D. Cote, 1989. Effect of long-term pig slurry and solid cattle manure application on soil chemical and biological properties. Canadian Journal of Soil Science 69: 39-47.

Ogg, A.G., 1982. Effect of activated carbon on phytotoxicity of terbacil to several crops. Weed Science. 30: 683-687.

Page, A.L., Elseewi, A.A., and I.R. Straughan, 1978. Physical and chemical properties of fly ash from coal-fired power plants with reference to environmental impacts. Residue Rev., (71):83-120.

Pawar, K. and P.S. Dubey, 1986. Growth response of maize in fly ash amended soils. Geobios 12: $53-56$.

Saint-Fort, R., and S.A. Visser, 1988. Study of the interactions between atrazine, diazinon and lindane with humic acids of various molecular weights. Journal of Environmental Science and Health, A23: 613-624.

Schnitzer, M., no date, Soil organic matter and soil quality. Humate Canada Limited Publication. p. 1-17.

Sharma, M.P., 1989. Efficiency of activated charcoal for inactivation of bromacil and tebuthiuron residues in soil. Alberta Land Conservation and Reclamation Council Report No. RRTAC 89-3. 38 pp.

Shea, P.J., 1985. Detoxification of herbicide residues in soil. Weed Science 33: 33-41.

Shoup, S.C. and C.E. Whitcomb, 1984. Activated charcoal does not prevent Hyvar X damage, American Nurseryman., Chicago, Ill., Am. Nurseryman Publish Co., 159: 41-43.

Stevenson, F.J., 1986. Cycles of soil: carbon, nitrogen, phosphorus, sulfur, micronutrients. A Wiley-Interscience publication. John Willey \& Sons. Toronto. p. 70-75.

Taylor, E.M, Jr., and G.E. Schuman, 1988. Fly ash and lime amendment of acidic coal spoil to aid revegetation. Journal of Environmental Quality 17: 120-124.

Upchurch, R.P. and D.D. Mason, 1962. The influence of soil organic matter on the phytotoxicity of herbicides. Weeds 10: 9-14. 
Wester, G.R., 1961. The effect of sawdust, straw, compost, and manure on the yield and chemical composition of strawberries and on soil moisture, acidity and organic matter content. Canadian Journal of Plant Science 41: 42-49.

Webster, G.R., Adamson, R.M., Crossley, J.H., Harris, J.H., Turley, R.H. and J.J. Woods, 1957. Sawdust for horticultural crops. Experimental Farm, Research Branch, Saanichton, B.C. Pub. No. 165.20 pp.

WSSA, 1989. Herbicide Handbook. Sixth edition. Weed Science Society of America, Champaign, Illinois. $301 \mathrm{pp}$.

Vandenbusch, M.B., and N.J. Sell, 1992. Fly ash as a sorbent for the removal of biologically resistant organic matter. Resources, Conservation and Recycling 6: 95-116. 


\section{RECLAMATION RESEARCH REPORTS}

1. RRTAC 79-2: Proceedings: Workshop on Native Shrubs in Reclamation. P.F. Ziemkiewicz, C.A. Dermott and H.P. Sims (Editors). 104 pp. No longer available.

The Workshop was organized as the first step in developing a Native Shrub reclamation research program. The Workshop provided a forum for the exchange of information and experiences on three topics: propagation; outplanting; and, species selection.

2. RRTAC 80-1: Test Plot Establishment: Native Grasses for Reclamation. R.S. Sadasivaiah and J. Weijer. 19 pp. No longer available.

The report details the species used at three test plots in Alberta's Eastern Slopes. Site preparation, experimental design, and planting method are also described.

3. RRTAC 80-2: Alberta's Reclamation Research Program - 1979. Reclamation Research Technical Advisory Committee. 22 pp. No longer available.

This report describes the expenditure of $\$ 1,190,006$ of Alberta Heritage Savings Trust Fund monies on research under the Land Reclamation Program. The report outlines the objectives and research strategies of the four program areas, and describes the projects funded under each program.

4. RRTAC 80-3: The Role of Organic Compounds in Salinization of Plains Coal Mining Sites.

N.S.C. Cameron et al. 46 pp. No longer available.

This is a literature review of the chemistry of sodic mine spoil and the changes expected to occur in groundwater.

5. RRTAC 80-4: Proceedings: Workshop on Reconstruction of Forest Soils in Reclamation.

P.F. Ziemkiewicz, S.K. Takyi and H.F. Regier (Editors). 160 pp. $\$ 10.00$

Experts in the field of forestry and forest soils report on research relevant to forest soil reconstruction and discuss the most effective means of restoring forestry capability of mined lands.

6. RRTAC 80-5: Manual of Plant Species Suitability for Reclamation in Alberta. L.E. Watson, R.W. Parker and D.F. Polster. 2 vols, 541 pp. No longer available; replaced by RRTAC 89-4.

Forty-three grass, fourteen forb, and thirty-four shrub and tree species are assessed in terms of their suitability for use in reclamation. Range maps, growth habit, propagation, tolerance, and availability information are provided.

7. RRTAC 81-1: The Alberta Government's Reclamation Research Program - 1980. Reclamation Research Technical Advisory Committee. 25 pp. No longer available.

This report describes the expenditure of $\$ 1,455,680$ of Alberta Heritage Savings Trust Fund monies on research under the Land Reclamation Program. The report outlines the objectives and research strategies of the four program areas, and describes the projects funded under each program. 
8. RRTAC 81-2: 1980 Survey of Reclamation Activities in Alberta. D.G. Walker and R.L. Rothwell. 76 pp. $\$ 10.00$

This survey is an update of a report prepared in 1976 on reclamation activities in Alberta, and includes research and operational reclamation, locations, personnel, etc.

9. RRTAC 81-3: Proceedings: Workshop on Coal Ash and Reclamation. P.F. Ziemkiewicz, R. Stein, R. Leitch and G. Lutwick (Editors). 253 pp. $\$ 10.00$

Presents nine technical papers on the chemical, physical, and engineering properties of Alberta fly and bottom ashes, revegetation of ash disposal sites, and use of ash as a soil amendment. Workshop discussions and summaries are also included.

10. RRTAC 82-1: Land Surface Reclamation: An International Bibliography. H.P. Sims and C.B. Powter. 2 vols, 292 pp. $\$ 10.00$

Literature to 1980 pertinent to reclamation in Alberta is listed in Vol. 1 and is also on the University of Alberta computing system (in a SPIRES database called RECLAIM). Vol. 2 comprises the keyword index and computer access manual.

11. RRTAC 82-2: A Bibliography of Baseline Studies in Alberta: Soils, Geology, Hydrology and Groundwater. C.B. Powter and H.P. Sims. 97 pp. $\$ 5.00$

This bibliography provides baseline information for persons involved in reclamation research or in the preparation of environmental impact assessments. Materials, up to date as of December 1981, are available in the Alberta Environment Library.

12. RRTAC 82-3: The Alberta Government's Reclamation Research Program - 1981. Reclamation Research Technical Advisory Committee. 22 pp. No longer available.

This report describes the expenditure of $\$ 1,499,525$ of Alberta Heritage Savings Trust Fund monies on research under the Land Reclamation Program. The report outlines the objectives and research strategies of the four program areas, and describes the projects funded under each program.

13. RRTAC 83-1: Soil Reconstruction Design for Reclamation of Oil Sand Tailings. Monenco Consultants Ltd. $185 \mathrm{pp}$. No longer available

Volumes of peat and clay required to amend oil sand tailings were estimated based on existing literature. Separate soil prescriptions were made for spruce, jack pine, and herbaceous cover types. The estimates form the basis of field trials (See RRTAC 92-4).

\section{RRTAC 83-2: The Alberta Government's Reclamation Research Program - 1982. Reclamation Research Technical Advisory Committee. $25 \mathrm{pp}$. No longer available.}

This report describes the expenditure of $\$ 1,536,142$ of Alberta Heritage Savings Trust Fund monies on research under the Land Reclamation Program. The report outlines the objectives and research strategies of the four program areas, and describes the projects funded under each program. 
15. RRTAC 83-3: Evaluation of Pipeline Reclamation Practices on Agricultural Lands in Alberta. Hardy Associates (1978) Ltd. 205 pp. No longer available.

Available information on pipeline reclamation practices was reviewed. A field survey was then conducted to determine the effects of pipe size, age, soil type, construction method, etc. on resulting crop production.

16. RRTAC 83-4: Proceedings: Effects of Coal Mining on Eastern Slopes Hydrology. P.F. Ziemkiewicz (Editor). 123 pp. $\$ 10.00$

Technical papers are presented dealing with the impacts of mining on mountain watersheds, their flow characteristics, and resulting water quality. Mitigative measures and priorities were also discussed.

17. RRTAC 83-5: Woody Plant Establishment and Management for Oil Sands Mine Reclamation. Techman Engineering Ltd. 124 pp. No longer available.

This is a review and analysis of information on planting stock quality, rearing techniques, site preparation, planting, and procedures necessary to ensure survival of trees and shrubs in oil sand reclamation.

18. RRTAC 84-1: Land Surface Reclamation: A Review of the International Literature. H.P. Sims, C.B. Powter and J.A. Camphell. 2 vols, 1549 pp. $\$ 20.00$

Nearly all topics of interest to reclamationists including mining methods, soil amendments, revegetation, propagation and toxic materials are reviewed in light of the international literature.

19. RRTAC 84-2: Propagation Study: Use of Trees and Shrubs for Oil Sand Reclamation. Techman Engineering Ltd. 58 pp. $\$ 10.00$

This report evaluates and summarizes all available published and unpublished information on large-scale propagation methods for shrubs and trees to be used in oil sand reclamation.

20. RRTAC 84-3: Reclamation Research Annual Report - 1983. P.F. Ziemkiewicz. 42 pp. $\$ 5.00$

This report describes the expenditure of $\$ 1,529,483$ of Alberta Heritage Savings Trust Fund monies on research under the Land Reclamation Program. The report outlines the objectives and research strategies of the four program areas and describes the projects funded under each program.

21. RRTAC 84-4: Soil Microbiology in Land Reclamation. D. Parkinson, R.M. Danielson, C. Griffiths, S. Visser and J.C. Zak. 2 vols, 676 pp. $\$ 10.00$

This is a collection of five reports dealing with re-establishment of fungal decomposers and mycorrhizal symbionts in various amended spoil types.

22. RRTAC 85-1: Proceedings: Revegetation Methods for Alberta's Mountains and Foothills. P.F. Ziemkiewicz (Editor). 416 pp. \$10.00.

Results of long-term experiments and field experience on species selection, fertilization, reforestation, topsoiling, shrub propagation and establishment are presented. 
23. RRTAC 85-2: Reclamation Research Annual Report - 1984. P.F. Ziemkiewicz. 29 pp. No longer available.

This report describes the expenditure of $\$ 1,320,516$ of Alberta Heritage Savings Trust Fund monies on research under the Land Reclamation Program. The report outlines the objectives and research strategies of the four program areas and describes the projects funded under each program.

24. RRTAC 86-1: A Critical Analysis of Settling Pond Design and Alternative Technologies. A. Somani. 372 pp. $\$ 10.00$

The report examines the critical issue of settling pond design, and sizing and alternative technologies. The study was co-funded with The Coal Association of Canada.

25. RRTAC 86-2: Characterization and Variability of Soil Reconstructed after Surface Mining in Central Alberta. T.M. Macyk. 146 pp. No longer available.

Reconstructed soils representing different materials handling and replacement techniques were characterized, and variability in chemical and physical properties was assessed. The data obtained indicate that reconstructed soil properties are determined largely by parent material characteristics and further tempered by materials handling procedures. Mining tends to create a relatively homogeneous soil landscape in contrast to the mixture of diverse soils found before mining.

26. RRTAC 86-3: Generalized Procedures for Assessing Post-Mining Groundwater Supply Potential in the Plains of Alberta - Plains Hydrology and Reclamation Project. M.R. Trudell and S.R. Moran. 30 pp. $\$ 5.00$

In the Plains region of Alberta, the surface mining of coal generally occurs in rural, agricultural areas in which domestic water supply requirements are met almost entirely by groundwater. Consequently, an important aspect of the capability of reclaimed lands to satisfy the needs of a residential component is the post-mining availability of groundwater. This report proposes a sequence of steps or procedures to identify and characterize potential postmining aquifers.

27. RRTAC 86-4: Geology of the Battle River Site: Plains Hydrology and Reclamation Project.

A. Maslowski-Schutze, R. Li, M. Fenton and S.R. Moran. 86 pp. $\$ 10.00$

This report summarizes the geological setting of the Battle River study site. It is designed to provide a general understanding of geological conditions adequate to establish a framework for hydrogeological and general reclamation studies. The report is not intended to be a detailed synthesis such as would be required for mine planning purposes.

28. RRTAC 86-5: Chemical and Mineralogical Properties of Overburden: Plains Hydrology and Reclamation Project. A. Maslowski-Schutze. 71 pp. $\$ 10.00$

This report describes the physical and mineralogical properties of overburden materials in an effort to identify individual beds within the bedrock overburden that might be significantly different in terms of reclamation potential. 
29. RRTAC 86-6: Post-Mining Groundwater Supply at the Battle River Site: Plains Hydrology and Reclamation Project. M.R. Trudell, G.J. Sterenberg and S.R. Moran. 49 pp. $\$ 5.00$

The report deals with the availability of water supply in or beneath cast overburden to support post-mining land use, including both quantity and quality considerations. The study area is in the Battle River Mining area in eastcentral Alberta.

30. RRTAC 86-7: Post-Mining Groundwater Supply at the Highvale Site: Plains Hydrology and Reclamation Project. M.R. Trudell. 25 pp. $\$ 5.00$

This report evaluates the availability of water supply in or beneath cast overburden to support post-mining land use, including both quantity and quality considerations. The study area is the Highvale mining area in west-central Alberta.

31. RRTAC 86-8: Reclamation Research Annual Report - 1985. P.F. Ziemkiewicz. 54 pp. $\$ 5.00$

This report describes the expenditure of $\$ 1,168,436$ of Alberta Heritage Savings Trust Fund monies on research under the Land Reclamation Program. The report outlines the objectives and research strategies of the four program areas and describes the projects funded under each program.

32. RRTAC 86-9: Wildlife Habitat Requirements and Reclamation Techniques for the Mountains and Foothills of Alberta. J.E. Green, R.E. Salter and D.G. Walker. 285 pp. No longer available.

This report presents a review of relevant North American literature on wildlife habitats in mountain and foothills biomes, reclamation techniques, potential problems in wildlife habitat reclamation, and potential habitat assessment methodologies. Four biomes (Alpine, Subalpine, Montane, and Boreal Uplands) and 10 key wildlife species (snowshoe hare, beaver, muskrat, elk, moose, caribou, mountain goat, bighorn sheep, spruce grouse, and whitetailed ptarmigan) are discussed. The study was co-funded with The Coal Association of Canada.

33. RRTAC 87-1: Disposal of Drilling Wastes. L.A. Leskiw, E. Reinl-Dwyer, T.L. Dabrowski, B.J. Rutherford and H. Hamilton. 210 pp. No longer available.

Current drilling waste disposal practices are reviewed and criteria in Alberta guidelines are assessed. The report also identifies research needs and indicates mitigation measures. A manual provides a decision-making flowchart to assist in selecting methods of environmentally safe waste disposal.

34. RRTAC 87-2: Minesoil and Landscape Reclamation of the Coal Mines in Alberta's Mountains and Foothills. A.W. Fedkenheuer, L.J. Knapik and D.G. Walker. 174 pp. No longer available.

This report reviews current reclamation practices with regard to site and soil reconstruction and re-establishment of biological productivity. It also identifies research needs in the Mountain-Foothills area. The study was cofunded with The Coal Association of Canada. 
35. RRTAC 87-3: Gel and Saline Drilling Wastes in Alberta: Workshop Proceedings. D.A. Lloyd (Compiler). 218 pp. No longer available.

Technical papers were presented which describe: mud systems used and their purpose; industrial constraints; government regulations, procedures and concerns; environmental considerations in waste disposal; and toxic constituents of drilling wastes. Answers to a questionnaire distributed to participants are included in an appendix.

36. RRTAC 87-4: Reclamation Research Annual Report - 1986. 50 pp. No longer available.

This report describes the expenditure of $\$ 1,186,000$ of Alberta Heritage Savings Trust Fund monies on research under the Land Reclamation Program. The report outlines the objectives and research strategies of the four program areas and describes the projects funded under each program.

37. RRTAC 87-5: Review of the Scientific Basis of Water Quality Criteria for the East Slope Foothills of Alberta. Beak Associates Consulting Ltd. 46 pp. $\$ 10.00$

The report reviews existing Alberta guidelines to assess the quality of water drained from coal mine sites in the East Slope Foothills of Alberta. World literature was reviewed within the context of the East Slopes environment and current mining operations. The ability of coal mine operators to meet the various guidelines is discussed. The study was co-funded with The Coal Association of Canada.

38. RRTAC 87-6: Assessing Design Flows and Sediment Discharge on the Eastern Slopes. Hydrocon Engineering (Continental) Ltd. and Monenco Consultants Ltd. 97 pp. $\$ 10.00$

The report provides an evaluation of current methodologies used to determine sediment yields due to rainfall events in well-defined areas. Models are available in Alberta to evaluate water and sediment discharge in a postmining situation. SEDIMOT II (Sedimentology Disturbed Modelling Techniques) is a single storm model that was developed specifically for the design of sediment control structures in watersheds disturbed by surface mining and is well suited to Alberta conditions. The study was co-funded with The Coal Association of Canada.

39. RRTAC 87-7: The Use of Bottom Ash as an Amendment to Sodic Spoil. S. Fullerton. 83 pp. No longer available.

The report details the use of bottom ash as an amendment to sodic coal mine spoil. Several rates and methods of application of bottom ash to sodic spoil were tested to determine which was the best at reducing the effects of excess sodium and promoting crop growth. Field trials were set up near the Vesta mine in East Central Alberta using ash readily available from a nearby coal-fired thermal generating station. The research indicated that bottom ash incorporated to a depth of $30 \mathrm{~cm}$ using a subsoiler provided the best results.

40. RRTAC 87-8: Waste Dump Design for Erosion Control. R.G. Chopiuk and S.E. Thornton. 45 pp. $\$ 5.00$

This report describes a study to evaluate the potential influence of erosion from reclaimed waste dumps on downslope environments such as streams and rivers. Sites were selected from coal mines in Alberta's mountains and foothills, and included resloped dumps of different configurations and ages, and having different vegetation covers. The study concluded that the average annual amount of surface erosion is minimal. As expected, erosion was greatest on slopes which were newly regraded. Slopes with dense grass cover showed no signs of erosion. Generally, the amount of erosion decreased with time, as a result of initial loss of fine particles, the formation of a weathered surface, and increased vegetative cover. 
41. RRTAC 87-9: Hydrogeology and Groundwater Chemistry of the Battle River Mining Area. M.R. Trudell, R.L. Faught and S.R. Moran. 97 pp. No longer available.

This report describes the premining geologic conditions in the Battle River coal mining area including the geology as well as the groundwater flow patterns, and the groundwater quality of a sequence of several water-bearing formations extending from the surface to a depth of about 100 metres.

42. RRTAC 87-10: Soil Survey of the Plains Hydrology and Reclamation Project - Battle River Project Area. T.M. Macyk and A.H. MacLean. 62 pp. plus 8 maps. $\$ 10.00$

The report evaluates the capability of post-mining landscapes and assesses the changes in capability as a result of mining, in the Battle River mining area. Detailed soils information is provided in the report for lands adjacent to areas already mined as well as for lands that are destined to be mined. Characterization of the reconstructed soils in the reclaimed areas is also provided. Data were collected from 1979 to 1985 . Eight maps supplement the report.

\section{RRTAC 87-11: Geology of the Highvale Study Site: Plains Hydrology and Reclamation Project.}

A. Maslowski-Schutze. 78 pp. $\$ 10.00$

The report is one of a series that describes the geology, soils and groundwater conditions at the Highvale Coal Mine study site. The purpose of the study was to establish a summary of site geology to a level of detail necessary to provide a framework for studies of hydrogeology and reclamation.

44. RRTAC 87-12: Premining Groundwater Conditions at the Highvale Site. M.R. Trudell and R. Faught. 83 pp. No longer available.

This report presents a detailed discussion of the premining flow patterns, hydraulic properties, and isotopic and hydrochemical characteristics of five layers within the Paskapoo Geological Formation, the underlying sandstone beds of the Upper Horseshoe Canyon Formation, and the surficial glacial drift.

\section{RRTAC 87-13: An Agricultural Capability Rating System for Reconstructed Soils. T.M. Macyk.} 27 pp. $\$ 5.00$

This report provides the rationale and a system for assessing the agricultural capability of reconstructed soils. Data on the properties of the soils used in this report are provided in RRTAC 86-2.

46. RRTAC 88-1: A Proposed Evaluation System for Wildlife Habitat Reclamation in the Mountains and Foothills Biomes of Alberta: Proposed Methodology and Assessment Handbook. T.R. Eccles, R.E. Salter and J.E. Green. 101 pp. plus appendix. $\$ 10.00$

The report focuses on the development of guidelines and procedures for the assessment of reclaimed wildlife habitat in the Mountains and Foothills regions of Alberta. The technical section provides background documentation including a discussion of reclamation planning, a listing of reclamation habitats and associated key wildlife species, conditions required for development, recommended revegetation species, suitable reclamation techniques, a description of the recommended assessment techniques and a glossary of basic terminology. The assessment handbook section contains basic information necessary for evaluating wildlife habitat reclamation, including assessment scoresheets for 15 different reclamation habitats, standard methodologies for measuring habitat variables used as assessment criteria, and minimum requirements for certification. This handbook is intended as a field manual that could potentially be used by site operators and reclamation officers. The study was co-funded with The Coal Association of Canada. 
47. RRTAC 88-2: Plains Hydrology and Reclamation Project: Spoil Groundwater Chemistry and its Impacts on Surface Water. M.R. Trudell (Compiler). 135 pp. No longer available.

Two reports comprise this volume. The first "Chemistry of Groundwater in Mine Spoil, Central Alberta, " describes the chemical make-up of spoil groundwater at four mines in the Plains of Alberta. It explains the nature and magnitude of changes in groundwater chemistry following mining and reclamation. The second report, "Impacts of Surface Mining on Chemical Quality of Streams in the Battle River Mining Area," describes the chemical quality of water in streams in the Battle River mining area, and the potential impact of groundwater discharge from surface mines on these streams.

48. RRTAC 88-3: Revegetation of Oil Sands Tailings: Growth Improvement of Silver-berry and Buffalo-berry by Inoculation with Mycorrhizal Fungi and $\mathrm{N}_{2}$-Fixing Bacteria. S. Visser and R.M. Danielson. 98 pp. $\$ 10.00$

The report provides results of a study: (1) To determine the mycorrhizal affinities of various actinorrhizal shrubs in the Fort McMurray, Alberta region; (2) To establish a basis for justifying symbiont inoculation of buffalo-berry and silver-berry; (3) To develop a growing regime for the greenhouse production of mycorrhizal, nodulated silverberry and buffalo-berry; and, (4) To conduct a field trial on reconstructed soil on the Syncrude Canada Limited oil sands site to critically evaluate the growth performance of inoculated silver-berry and buffalo-berry as compared with their un-inoculated counterparts.

49. RRTAC 88-4: Plains Hydrology and Reclamation Project: Investigation of the Settlement Behaviour of Mine Backfill. D.R. Pauls (compiler). 135 pp. \$10.00

This three part volume covers the laboratory assessment of the potential for subsidence in reclaimed landscapes. The first report in this volume, "Simulation of Mine Spoil Subsidence by Consolidation Tests, " covers laboratory simulations of the subsidence process particularly as it is influenced by resaturation of mine spoil. The second report, "Water Sensitivity of Smectitic Overburden: Plains Region of Alberta," describes a series of laboratory tests to determine the behaviour of overburden materials when brought into contact with water. The report entitled "Classification System for Transitional Materials: Plains Region of Alberta, " describes a lithological classification system developed to address the characteristics of the smectite rich, clayey transition materials that make up the overburden in the Plains of Alberta.

\section{RRTAC 88-5: Ectomycorrhizae of Jack Pine and Green Alder: Assessment of the Need for Inoculation, Development of Inoculation Techniques and Outplanting Trials on Oil Sand Tailings. R.M. Danielson and S. Visser. 177 pp. No longer available.}

The overall objective of this research was to characterize the mycorrhizal status of Jack Pine and Green Alder which are prime candidates as reclamation species for oil sand tailings and to determine the potential benefits of mycorrhizae on plant performance. This entailed determining the symbiont status of container-grown nursery stock and the quantity and quality of inoculum in reconstructed soils, developing inoculation techniques and finally, performance testing in an actual reclamation setting.

\section{RRTAC 88-6: Reclamation Research Annual Report - 1987. Reclamation Research Technical} Advisory Committee. 67 pp. No longer available.

This annual report describes the expenditure of $\$ 500,000.00$ of Alberta Heritage Savings Trust Fund monies on research under the Land Reclamation Program. The report outlines the objectives and research strategies of the four program areas, and describes the projects funded under each program. 
52. RRTAC 88-7: Baseline Growth Performance Levels and Assessment Procedure for Commercial Tree Species in Alberta's Mountains and Foothills. W.R. Dempster and Associates Ltd. 66 pp. $\$ 5.00$

Data on juvenile height development of lodgepole pine and white spruce from cut-over or burned sites in the Eastern Slopes of Alberta were used to define reasonable expectations of early growth performance as a basis for evaluating the success of reforestation following coal mining. Equations were developed predicting total seedling height and current annual height increment as a function of age and elevation. Procedures are described for applying the equations, with further adjustments for drainage class and aspect, to develop local growth performance against these expectations. The study was co-funded with The Coal Association of Canada.

\section{RRTAC 88-8: Alberta Forest Service Watershed Management Field and Laboratory Methods.} A.M.K. Nip and R.A. Hursey. 4 Sections, various pagings. $\$ 10.00$

Disturbances such as coal mines in the Eastern Slopes of Alberta have the potential for affecting watershed quality during and following mining. The collection of hydrometric, water quality and hydrometeorologic information is a complex task. A variety of instruments and measurement methods are required to produce a record of hydrologic inputs and outputs for a watershed basin. There is a growing awareness and recognition that standardization of data acquisition methods is required to ensure data comparability, and to allow comparison of data analyses. The purpose of this manual is to assist those involved in the field of data acquisition by outlining methods, practices and instruments which are reliable and recognized by the International Organization for Standardization.

\section{RRTAC 88-9: Computer Analysis of the Factors Influencing Groundwater Flow and Mass Transport in a System Disturbed by Strip Mining. F.W. Schwartz and}

A.S. Crowe. $78 \mathrm{pp}$. No longer available.

Work presented in this report demonstrates how a groundwater flow model can be used to study a variety of mining-related problems such as declining water levels in areas around the mine as a result of dewatering, and the development of high water tables in spoil once resaturation is complete. This report investigates the role of various hydrogeological parameters that influence the magnitude, timing, and extent of water level changes during and following mining at the regional scale. The modelling approach described here represents a major advance on existing work.

\section{RRTAC 88-10: Review of Literature Related to Clay Liners for Sump Disposal of Drilling Wastes.} D.R. Pauls, S.R. Moran and T. Macyk. 61 pp. No longer available.

The report reviews and analyses the effectiveness of geological containment of drilling waste in sumps. Of particular importance was the determination of changes in properties of clay materials as a result of contact with highly saline brines containing various organic chemicals.

\section{RRTAC 88-11: Highvale Soil Reconstruction Project: Five Year Summary. D.N. Graveland,} T.A. Oddie, A.E. Osborne and L.A. Panek. 104 pp. $\$ 10.00$

This report provides details of a five year study to determine a suitable thickness of subsoil to replace over minespoil in the Highvale plains coal mine area to ensure return of agricultural capability. The study also examined the effect of slope and aspect on agricultural capability. This study was funded and managed with industry assistance. 
57. RRTAC 88-12: A Review of the International Literature on Mine Spoil Subsidence. J.D. Scott, G. Zinter, D.R. Pauls and M.B. Dusseault. 36 pp. $\$ 10.00$

The report reviews available engineering literature relative to subsidence of reclaimed mine spoil. The report covers methods for site investigation, field monitoring programs and lab programs, mechanisms of settlement, and remedial measures.

\section{RRTAC 89-1: Reclamation Research Annual Report - 1988. 74 pp. $\$ 5.00$}

This annual report describes the expenditure of $\$ 280,000.00$ of Alberta Heritage Savings Trust Fund monies on research under the Land Reclamation Program. The report outlines the objectives and research strategies of the four program areas, and describes the projects funded under each program.

59. RRTAC 89-2: Proceedings of the Conference: Reclamation, A Global Perspective. D.G. Walker, C.B. Powter and M.W. Pole (Compilers). 2 Vols., 854 pp. No longer available.

Over 250 delegates from all over the world attended this conference held in Calgary in August, 1989. The proceedings contains over 85 peer-reviewed papers under the following headings: A Global Perspective; Northern and High Altitude Reclamation; Fish \& Wildlife and Rangeland Reclamation; Water; Herbaceous Revegetation; Woody Plant Revegetation and Succession; Industrial and Urban Sites; Problems and Solutions; Sodic and Saline Materials; Soils and Overburden; Acid Generating Materials; and, Mine Tailings.

60. RRTAC 89-3: Efficiency of Activated Charcoal for Inactivation of Bromacil and Tebuthiuron Residues in Soil. M.P. Sharma. 38 pp. ISBN 0-7732-0878-X. \$5.00

Bromacil and Tebuthiuron were commonly used soil sterilants on well sites, battery sites and other industrial sites in Alberta where total vegetation control was desired. Activated charcoal was found to be effective in binding the sterilants in greenhouse trials. The influence of factors such as herbicide:charcoal concentration ratio, soil texture, organic matter content, soil moisture, and the time interval between charcoal incorporation and plant establishment were evaluated in the greenhouse.

61. RRTAC 89-4: Manual of Plant Species Suitability for Reclamation in Alberta - 2nd Edition. Hardy BBT Limited. 436 pp. ISBN 0-7732-0882-8. \$10.00.

This is an updated version of RRTAC Report 80-5 which describes the characteristics of 43 grass, 14 forb and 34 shrub and tree species which make them suitable for reclamation in Alberta. The report has been updated in several important ways: a line drawing of each species has been added; the range maps for each species have been redrawn based on an ecosystem classification of the province; new information (to 1990) has been added, particularly in the sections on reclamation use; and the material has been reorganized to facilitate information retrieval. Of greatest interest is the performance chart that precedes each species and the combined performance charts for the grass, forb, and shrub/tree groups. These allow the reader to pick out at a glance species that may suit their particular needs. The report was produced with the assistance of a grant from the Recreation, Parks and Wildlife Foundation.

\section{RRTAC 89-5: Battle River Soil Reconstruction Project Five Year Summary. L.A. Leskiw.} 188 pp. No longer available.

This report summarizes the results of a five year study to investigate methods required to return capability to land surface mined for coal in the Battle River area of central Alberta. Studies were conducted on: the amounts of subsoil required, the potential of gypsum and bottom ash to amend adverse soil properties, and the effects of slope angle and aspect. Forage and cereal crop growth was evaluated, as were changes in soil chemistry, density and moisture holding characteristics. 
63. RRTAC 89-6: Detailed Sampling, Characterization and Greenhouse Pot Trials Relative to Drilling Wastes in Alberta. T.M. Macyk, F.I. Nikiforuk, S.A. Abboud and Z.W. Widtman. 228 pp. No longer available.

This report summarizes a three-year study of the chemistry of freshwater gel, $\mathrm{KCl}, \mathrm{NaCl}, \mathrm{DAP}$, and invert drilling wastes, both solids and liquids, from three regions in Alberta: Cold Lake, Eastern Slopes, and Peace River/Grande Prairie. A greenhouse study also examined the effects of adding various amounts of waste to soil on grass growth and soil chemistry. Methods for sampling drilling wastes are recommended.

\section{RRTAC 89-7: A User's Guide for the Prediction of Post-Mining Groundwater Chemistry from} Overburden Characteristics. M.R. Trudell and D.C. Cheel. 55 pp. $\$ 5.00$

This report provides the detailed procedure and methodology that is required to produce a prediction of post-mining groundwater chemistry for plains coal mines, based on the soluble salt characteristics of overburden materials. The fundamental component of the prediction procedure is the geochemical model PHREEQE, developed by the U.S. Geological Survey, which is in the public domain and has been adapted for use on personal computers.

\section{RRTAC 90-1: Reclamation Research Annual Report - 1989. 62 pp. No longer available.}

This annual report describes the expenditure of $\$ 480,000.00$ of Alberta Heritage Savings Trust Fund monies on research under the Land Reclamation Program. The report outlines the objectives and research strategies of the four program areas, and describes the projects funded under each program.

66. RRTAC 90-2: Initial Selection for Salt Tolerance in Rocky Mountain Accessions of Slender Wheatgrass and Alpine Bluegrass. R. Hermesh, J. Woosaree, B.A. Darroch, S.N. Acharya and A. Smreciu. 40 pp. $\$ 5.00$

Selected lines of slender wheatgrass and alpine bluegrass collected from alpine and subalpine regions of Alberta as part of another native grass project were evaluated for their ability to emerge in a saline medium. Eleven slender wheatgrass and 72 alpine bluegrass lines had a higher percentage emergence than the Orbit Tall Wheatgrass control (a commonly available commercial grass). This means that as well as an ability to grow in high elevation areas, these lines may also be suitable for use in areas where saline soil conditions are present. Thus, their usefulness for reclamation has expanded.

\section{RRTAC 90-3: Natural Plant Invasion into Reclaimed Oil Sands Mine Sites. Hardy BBT Limited.} 65 pp. $\$ 5.00$

Vegetation data from reclaimed sites on the Syncrude and Suncor oil sands mines have been summarized and related to site and factors and reclamation methods. Natural invasion into sites seeded to agronomic grasses and legumes was minimal even after 15 years. Invasion was slightly greater in sites seeded to native species, but was greatest on sites that were not seeded. Invasion was mostly from agronomic species and native forbs; native shrub and tree invasion was minimal. 
68. RRTAC 90-4: Physical and Hydrological Characteristics of Ponds in Reclaimed Upland Landscape Settings and their Impact on Agricultural Capahility. S.R. Moran, T.M. Macyk, M.R. Trudell and M.E. Pigot, Alberta Research Council. 76 pp. $\$ 5.00$

The report details the results and conclusions from studying a pond in a reclaimed upland site in Vesta Mine. The pond formed as a result of two factors: (1) a berm which channelled meltwater into a series of subsidence depressions, forming a closed basin; and (2) low hydraulic conductivity in the lower subsoil and upper spoil as a result of compaction during placement and grading which did not allow for rapid drainage of ponded water. Ponds such as this in the reclaimed landscape can affect agricultural capability by: (1) reducing the amount of farmable land (however, the area covered by these ponds in this region is less than half of that found in unmined areas); and, (2) creating the conditions necessary for the progressive development of saline and potentially sodic soils in the area adjacent to the pond.

69. RRTAC 90-5: Review of the Effects of Storage on Topsoil Quality. Thurber Consultants Ltd., Land Resources Network Ltd., and Norwest Soil Research Ltd. 116 pp. $\$ 10.00$

The international literature was reviewed to determine the potential effects of storage on topsoil quality. Conclusions from the review indicated that storage does not appear to have any severe and longterm effects on topsoil quality. Chemical changes may be rectified with the use of fertilizers or manure. Physical changes appear to be potentially less serious than changes in soil quality associated with the stripping and respreading operations. Soil biotic populations appear to revert to pre-disturbance levels of activity within acceptable timeframes. Broad, shallow storage piles that are seeded to acceptable grass and legume species are recommended; agrochemical use should be carefully controlled to ensure soil biota are not destroyed.

\section{RRTAC 90-6: Proceedings of the Industry/Government Three-Lift Soils Handling Workshop.} Deloitte \& Touche. 168 pp. $\$ 10.00$

This report documents the results of a two-day workshop on the issue of three-lift soils handling for pipelines. The workshop was organized and funded by RRTAC, the Canadian Petroleum Association and the Independent Petroleum Association of Canada. Day one focused on presentation of government and industry views on the criteria for three-lift, the rationale and field data in support of three- and two-lift procedures, and an examination of the various soil handling methods in use. During day two, five working groups discussed four issues: alternatives to three-lift; interim criteria and suggested revisions; research needs; definitions of terms. The results of the workshop are being used by a government/industry committee to revise soils handling criteria for pipelines.

\section{RRTAC 90-7: Reclamation of Disturbed Alpine Lands: A Literature Review. Hardy BBT Limited. 209 pp. $\$ 10.00$}

This review covers current information from North American sources on measures needed to reclaim alpine disturbances. The review provides information on pertinent Acts and regulations with respect to development and environmental protection of alpine areas. It also discusses: alpine environmental conditions; current disturbances to alpine areas; reclamation planning; site and surface preparation; revegetation; and, fertilization. The report also provides a list of research and information needs for alpine reclamation in Alberta.

\section{RRTAC 90-8: Plains Hydrology and Reclamation Project: Summary Report. S.R. Moran,} M.R. Trudell, T.M. Macyk and D.B. Cheel. 105 pp. $\$ 10.00$

This report summarizes a 10-year study on the interactions of groundwater, soils and geology as they affect successful reclamation of surface coal mines in the plains of Alberta. The report covers: Characterization of the Battle River and Wabamun study areas; Properties of reclaimed materials and landscapes; Impacts of mining and reclamation on post-mining land use; and, Implications for reclamation practice and regulation. This project has led to the publication of 18 RRTAC reports and 22 papers in conference proceedings and referred journals. 
73. RRTAC 90-9: Literature Review on the Disposal of Drilling Waste Solids. Monenco Consultants Limited. 83 pp. $\$ 5.00$

This report reviews the literature on, and government and industry experience with, burial of drilling waste solids in an Alberta context. The review covers current regulations in Alberta, other provinces, various states in the US and other countries. Definitions of various types of burial are provided, as well as brief summaries of other possible disposal methods. Environmental concerns with the various options are presented as well as limited information on costs and monitoring of burial sites. The main conclusion of the work is that burial is still a viable option for some waste types but that each site and waste type must be evaluated on its own merits.

\section{RRTAC 90-10: Potential Contamination of Shallow Aquifers by Surface Mining of Coal.} M.R. Trudell, S.R. Moran and T.M. Macyk. 75 pp. $\$ 5.00$

This report presents the results of a field investigation of the movement of salinized groundwater from a mined and reclaimed coal mine near Forestburg into an adjacent unmined area. The movement is considered to be an unusual occurrence resulting from a combination of a hydraulic head that is higher in the mined area than in the adjacent coal aquifer, and the presence of a thin surficial sand aquifer adjacent to the mine. The high hydraulic head results from deep ponds in the reclaimed landscape that recharge the base of the spoil.

\section{RRTAC 91-1: Reclamation Research Annual Report - 1990. Reclamation Research Technical Advisory Committee. 69 pp. No longer available.}

This annual report describes the expenditure of \$499 612 of Alberta Heritage Savings Trust Fund monies on research under the Land Reclamation Program. The report outlines the objectives and research strategies of the four program areas, and describes the projects funded under each program. The report lists the 70 research reports published under the program.

\section{RRTAC 91-2: Winter Soil Evaluation and Mapping for Regulated Pipelines. A.G. Twardy.} 43 pp. ISBN 0-7732-0874-7. $\$ 5.00$

Where possible, summer soil evaluations are preferred for pipelines. However, when winter soil evaluations must be done, this report lays out the constraints and requirements for obtaining the best possible information. Specific recommendations include: restricting evaluations to the time of day with the best light conditions; use of core- or auger-equipped drill-trucks; increased frequency of site inspections and soil analyses; and, hiring a well-qualified pedologist. The province's soils are divided into four classes, based on their difficulty of evaluation in winter: slight (most soils); moderate; high; and, severe (salt-affected soils in the Brown and Dark Brown Soil Zones).

\section{RRTAC 91-3: A User Guide to Pit and Quarry Reclamation in Alberta. J.E. Green, T.D. Van Egmond, C. Wylie, I. Jones, L. Knapik and L.R. Paterson. 151 pp. ISBN 0-7732-0876-3. \$10.00}

Sand and gravel pits or quarries are usually reclaimed to the original land use, especially if that was better quality agricultural or forested land. However, there are times when alternative land uses are possible. This report outlines some of the alternate land uses for reclaimed sand and gravel pits or quarries, including: agriculture, forestry, wildlife habitat, fish habitat, recreation, and residential and industrial use. The report provides a general introduction to the industry and to the reclamation process, and then outlines some of the factors to consider in selecting a land use and the methods for reclamation. The report is not a detailed guide to reclamation; it is intended to help an operator determine if a land use would be suitable and to guide him or her to other sources of information. 
78. RRTAC 91-4: Soil Physical Properties in Reclamation. M.A. Naeth, D.J. White, D.S. Chanasyk, T.M. Macyk, C.B. Powter and D.J. Thacker. 204 pp. ISBN 0-7732-0880-1. \$10.00

This report provides information from the literature and Alberta sources on a variety of soil physical properties that can be measured on reclaimed sites. Each property is explained, measurement methods, problems, level of accuracy and common soil values are presented, and methods of dealing with the property (prevention, alleviation) are discussed. The report also contains the results of a workshop held to discuss soil physical properties and the state-of-the-art in Alberta.

79. RRTAC 92-1: Reclamation of Sterilant Affected Sites: A Review of the Issue in Alberta. M. Cotton and M.P. Sharma. 64 pp. ISBN 0-7732-0884-4. No longer available

This report assesses the extent of sterilant use on oil and gas leases in Alberta, identifies some of the concerns related to reclamation of sterilant affected sites and the common methods for reclaiming these sites, and outlines the methods for sampling and analyzing soils from sterilant affected sites. The report also provides an outline of a research program to address issues raised by government and industry staff.

80. RRTAC 92-2: Reclamation Research Annual Report - 1991. Reclamation Research Technical Advisory Committee. 55 pp. ISBN 0-7732-0888-7. No longer available.

This report describes the expenditure of $\$ 485,065$ of Alberta Heritage Savings Trust Fund monies on research under the Land Reclamation Program. The report outlines the objectives and research strategies of the five program areas, and describes the projects funded under each program. It also lists the 75 research reports that have been published to date.

\section{RRTAC 92-3: Proceedings of the Industry/Government Pipeline Reclamation Success Measurement Workshop. R.J. Mahnic and J.A. Toogood. 62 pp. ISBN 0-7732-0886-0. \$5.00.}

This report presents the results of a workshop to identify the soil and vegetation parameters that should be used to assess reclamation success on pipelines in Alberta. Six soil parameters (topsoil admixing; topsoil replacement thickness; compaction; soil loss by erosion; texture; and salinity) and six vegetation parameters (plant density; species composition; ground cover; vigour; weeds/undesirable species; and rooting characteristics) were selected as most important. Working groups discussed these parameters and presented suggested methods for assessing them in the field.

\section{RRTAC 92-4: Oil Sands Soil Reconstruction Project Five Year Summary. HBT AGRA Limited. 109 pp. ISBN 0-7732-0875-5. \$10.00}

This report documents a five year study of the effects of clay and peat amendments to oil sand tailings sand on survival and growth of trees and shrubs. Ten species (jack pine, white spruce, serviceberry, silverberry, buffaloberry, pin cherry, prickly/woods rose, Northwest poplar, green alder, and Bebb willow) were planted into tailings sand amended with three levels of peat and three levels of clay. The treatments were incorporated to a depth of $20 \mathrm{~cm}$ or $40 \mathrm{~cm}$. Data are provided on plant survival and growth, root size and distribution, disease and small mammal damage, herbaceous cover, soil moisture, soil chemistry, and bulk density. 
83. RRTAC 92-5: A Computer Program to Simulate Groundwater Flow and Contaminant Transport in the Vicinity of Active and Reclaimed Strip Mines: A User's Guide. A.S. Crowe and F.W. Schwartz, SIMCO Groundwater Research Ltd. 104 pp. plus appendix. ISBN 0-7732-0877-1. NOTE: This report is only available from the Alberta Research Council, Publications Centre, 250 Karl Clark Road, P.O. Box 8330, Station F, EDMONTON, Alberta T6H 5R7 as ARC Information Series 119. The cost is \$20.00 and the cheque must be made out to the Alberta Research Council.

The manual describes a computer program that was developed to study the influence of coal strip mining on groundwater flow systems and to simulate the transport of generated contaminants, both spatially and in time, in the vicinity of a mine. All three phases of a strip mine can be simulated: the pre-mining regional groundwater flow system; the mining and reclamation phase; and, the post-mining water level readjustment phase. The model is sufficiently general to enable the user to specify virtually any type of geological conditions, mining scenario, and boundary conditions.

84. RRTAC 92-6: Alberta Drilling Waste Sump Chemistry Study. Volume I: Report (Volume II: Appendices is only available through the Alberta Research Council, Publications Centre, 250 Karl Clark Road, P.O. Box 8330, Station F, EDMONTON, Alberta T6H 5R7. The cost is $\$ 15.00$ and the cheque must be made out to the Alberta Research Council.). T.M. Macyk, S.A. Abboud and F.I. Nikiforuk, Alberta Research Council. 217 pp. ISBN 0-7732-0879-8. \$10.00.

This study synthesizes the data from sampling and analysis of the solids and liquids found in 128 drilling waste sumps across Alberta. Drilling waste types sampled included: 72 freshwater gel, 19 invert, $27 \mathrm{KCl}, 2 \mathrm{NaCl}$, and 8 others. Data and statistics are tabulated by waste type, depth of the drill hole, and ERCB administrative region for both the solids and the liquids. Using preliminary loading limits developed by the government/industry Drilling Waste Review Committee, the report presents information on the volume and depth of waste that could be landspread, and the area required for landspreading. The oil and gas industry provided approximately $\$ 585,000$ for the sampling and analysis phase of this study.

85. RRTAC 93-1: Reclamation of Native Grasslands in Alberta: A Review of the Literature. D.S. Kerr, L.J. Morrison and K.E. Wilkinson, Environmental Management Associates. 205 pp. plus appendices. ISBN 0-7732-0881-X. \$10.00.

A review of the literature on native grassland reclamation was conducted to summarize the current state of knowledge on reclamation and restoration efforts within Alberta. The review is comprehensive, including an overview of the regulations and guidelines governing land use on native prairie; a description of the dominant grassland ecoregions in Alberta; a review of the common disturbance types, extent and biophysical effects of disturbance on native prairie within Alberta; a description of the factors which influence the degree of disturbance and reclamation; and examples of both natural and enhanced recovery of disturbed sites through the examination of selected case studies.

\section{RRTAC 93-2: Reclamation Research Annual Report - 1992. Reclamation Research Technical Advisory Committee. 56 pp. ISBN 0-7732-0883-6. \$5.00.}

This report describes the expenditure of $\$ 474,705$ of Alberta Heritage Savings Trust Fund monies on research under the Land Reclamation Program. The report outlines the objectives and the research strategies of the five programs, and describes the projects funded under each program. It also lists the 85 research reports that have been published to date. 
87. RRTAC 93-3: Catalogue of Technologies for Reducing the Environmental Impact of Fine Tailings from Oil Sand Processing. B.J. Fuhr, Alberta Research Council, D.E. Rose, Dereng Enterprises Ltd., and D. Taplin, Komex International Ltd. 63 pp. ISBN 0-7732-0885-2. \$5.00.

A catalogue containing 22 technologies for reducing the environmental impact of fine tailings derived from oil sands has been assembled. The report consists of an introduction to oil sand processing and fine tailings generation, a simple spreadsheet for comparing the technologies, and a process summary for each technology. The technologies were not evaluated for effectiveness. Rather, a detailed set of questions was prepared that highlights the environmentally-related information a proponent should have. These questions will help to form a basis for comparisons among the technologies.

88. RRTAC 93-4: Organic Materials as Soil Amendments in Reclamation: A Review of the Literature. Land Resources Network Ltd. 228 pp. ISBN 0-7732-0887-9. \$10.00

A review of the literature was conducted to examine the effect of various organic materials when used as amendments to disturbed soil. Organic amendments reviewed included animal manures, crop residues, peat, wood wastes, sewage sludge, municipal yard waste, humates, vermicomposts, and spent mushroom composts. Their effects on soil chemistry, physical properties, and biology were examined. Application methods, costs, longevity of effects, and use in reclamation were also reviewed. Benefits and drawbacks of each were discussed.

89. RRTAC 93-5: Drilling Waste Disposal. T.M. Macyk and S.A. Abboud, Alberta Research Council. 125 pp. ISBN 0-7732-0889-5. $\$ 10.00$

An overall perspective and description of the steps involved in the management and land-based disposal of drilling wastes in Alberta. A computer program, available from the Alberta Research Council, has been written to support the data management required for proper disposal. A field manual is in preparation. These three information sources provide technical support for the Energy Resources Conservation Board's Guide G-50: Drilling Waste Management.

90. RRTAC 93-6: Mapping and Characterization of Cutover Peatlands for Reclamation Planning. L.W. Turchenek, Alberta Research Council, W.S. Tedder, Alberta Agriculture, Food and Rural Development, and R. Krzanowski, Alberta Research Council. 100 pp. ISBN 0-7732-6038-2. $\$ 5.00$

The report presents a methodology for cost-effective soil survey and sampling of cutover peatlands. It also presents baseline chemical information and data interpretation for peat materials from a cutover peatland site. The report provides background information on classifying and describing peatlands. This information can be used to develop reclamation plans.

91. RRTAC 93-7: Soil Series Information for Reclamation Planning in Alberta. Pedocan Land Evaluation Ltd. Various pagings. ISBN 0-7732-6041-2. \$10.00

This manual has been published to provide conservation and reclamation planners with information and guidelines to help understand and use soil inventory data. The soil series in the manual correspond to those in the Generation 2 Alberta Soil Names File. Part 1 of the manual describes the terminology used in soil surveys and presents the assumptions and conventions upon which the interpretations for each soil series are based. Part 2 presents typical data and interpretations for each soil series. 
92. RRTAC 93-8: Oil Sands Sludge Dewatering by Freeze-Thaw and Evapotranspiration. R.L. Johnson, P. Bork. W. H. James and L. Koverny, Alberta Environmental Centre. 247 pp. ISBN 0-7732-6042-0. $\$ 10.00$

This report presents data from a series of laboratory and field experiments designed to evaluate the removal of water from oil sands sludge. A number of plant species were evaluated and two, reed canary grass and western dock, were found to remove a significant amount of water through evapotranspiration. Freeze-thaw cycles were also found to remove water from both sand-sludge mixtures and pure sludge. A combination of freeze-thaw and biological dewatering using plants was found to increase solids content from $30 \%$ to $80 \%$. At $80 \%$ solids the sludge had a shear strength of $120 \mathrm{kPa}$ and could support machine traffic. These studies prompted further field work.

93. RRTAC 93-9: Native Legumes for Reclamation in Alberta. A. Smreciu, Wild Rose Consulting Inc. 94 pp. ISBN 0-7732-0643-9. \$5.00

Seeds from Astragalus (milkvetches), Hedysarum (sweetbrooms), Lupinus (lupins), and Oxytropis (locoweeds) were collected from the mountains and foothills region of Alberta, from Waterton Lakes National Park to Grande Cache. The species were tested for germination and seedlings were established and evaluated for three growing seasons in Vegreville. The species were evaluated based on survival, growth and development, and yield. Astragalus alpinus was selected as the most promising species. Oxytropis monticola and Oxytropis splendens were also recommended.

94. RRTAC 93-10: Proceedings of the Alberta Wellsite Reclamation Criteria Workshop. R.J. Mahnic, Communiplan Inc., L.J. Knapik and T.R. Bossenberry, Pedocan Land Evaluation Ltd., and G.C. Mott, G.C. Mott Associates. Various Pagings. ISBN 0-7732-0644-7. $\$ 10.00$

This report summarizes government, industry and public comments received before and during a two-day workshop held to discuss the Reclamation Criteria for Wellsites and Associated Facilities. The information in the report was used to revise the Criteria for use from 1994 onward.

95. RRTAC 93-11: Salt Movement in Disturbed Soils. N.M. Finlayson, Land Resources Network. 61pp. ISBN 0-7732-6045-5. $\$ 5.00$

The report reviews the literature regarding the movement of salts down or up in the soil profile follwing surface disturbance. The objective was to find out if salts in the upper $50 \mathrm{~cm}$ of a soil profile would return to pre-disturbance levels. If they do, special soil handling requirements may not be needed for saline soils when pipelining. There were few studies on pipelines so the majority of the report focuses on mining and deep plowing studies. The results varied between soil zones. More pipeline specific work is required. 
